\title{
WestVirginiaUniversity
}

THE RESEARCH REPOSITORY @ WVU

Graduate Theses, Dissertations, and Problem Reports

2009

\section{Measuring and changing negative stuttering stereotypes in adolescents}

Timothy W. Flynn

West Virginia University

Follow this and additional works at: https://researchrepository.wvu.edu/etd

\section{Recommended Citation}

Flynn, Timothy W., "Measuring and changing negative stuttering stereotypes in adolescents" (2009). Graduate Theses, Dissertations, and Problem Reports. 2759.

https://researchrepository.wvu.edu/etd/2759

This Thesis is protected by copyright and/or related rights. It has been brought to you by the The Research Repository @ WVU with permission from the rights-holder(s). You are free to use this Thesis in any way that is permitted by the copyright and related rights legislation that applies to your use. For other uses you must obtain permission from the rights-holder(s) directly, unless additional rights are indicated by a Creative Commons license in the record and/ or on the work itself. This Thesis has been accepted for inclusion in WVU Graduate Theses, Dissertations, and Problem Reports collection by an authorized administrator of The Research Repository @ WVU. For more information, please contact researchrepository@mail.wvu.edu. 
Measuring and Changing Negative Stuttering Stereotypes in Adolescents

Timothy W. Flynn

Thesis submitted to the College of Human Resources and Education at West Virginia University in partial fulfillment of the requirements for the degree of Master of Science

in

Speech-Language Pathology

\author{
Kenneth O. St. Louis, Ph.D., Chair
}

Norman J. Lass, Ph.D.

Dennis M. Ruscello, Ph.D.

Department of Speech Pathology and Audiology

Morgantown, West Virginia

2009

Keywords: Stuttering; Stereotype; Attitudes; Changing; Adolescents

Copyright 2009 Timothy W. Flynn 


\section{ABSTRACT \\ Measuring and Changing Negative Stuttering Stereotypes in Adolescents}

\section{Timothy W. Flynn}

Stuttering is known to carry stereotypes, e.g., that people who stutter are nervous, anxious, and shy. Research has shown that negative stereotypes about people who stutter exist within the general population. Moreover, negative stereotypes exist among teachers, students, speechlanguage pathologists, and even the parents of people who stutter. While public opinion of stuttering does not vary dramatically between populations, the ways in which we might change it does. Research has shown that educational videos, books, and classes about stuttering do not appear to significantly alter public opinion positively.

This study compared the effectiveness of two forms of advocacy, a live presentation versus a video presentation, in altering the stuttering stereotype among adolescents. Participants filled out a questionnaire before they were exposed to a 45-minute live presentation or a 45 -minute video presentation. After these live and video presentations, they completed a second questionnaire. Then, participants who watched the video were exposed to a shortened 20-minute live presentation followed by a third and final questionnaire.

Results indicated that adolescents showed evidence of negative stereotypes towards people who stutter prior to the presentations. Overall, there were 27 significant positive attitude changes towards stuttering ( $\mathrm{p}$ 0.005), 15 for live presentations and 12 for video presentations. Five additional significant positive attitude changes occurred after the video presentation as a result of the shortened live presentation. These findings demonstrate that adolescents' opinions on stuttering can be altered in a positive direction. A live presentation appears to have a greater positive impact on altering attitudes than a video presentation, and moreover, a shortened live presentation following a video presentation can further alter attitudes positively. 


\section{ACKNOWLEDGEMENTS}

Writing this thesis has been the largest undertaking of my academic career. I would like to thank the members of my committee, Dr. Norman Lass and Dr. Dennis Ruscello for putting in the time and effort to read through my entire thesis, and make recommendations to best help me. Dr. Kenneth St. Louis has served as my committee chair, senior capstone mentor, clinical supervisor, advisor, and friend. Dr. St. Louis and I met 6 years ago by chance when my English teacher recommended I speak to him regarding a research paper I was writing on stuttering. After a few meetings, he encouraged me to attend group therapy, speech therapy, and through a lot of time and consideration, helped me to switch my major to speech pathology. Through his guidance I began speaking to college and high school level classes about stuttering, which in turn, created the foundation for my interest in altering negative attitudes about stuttering. He helped me tremendously with my senior capstone paper, which not only was presented at the annual convention of American Speech-Language Hearing Association as a poster session, but aided me greatly in being accepted into graduate school in 2007. Since entering graduate school, he has been a constant help and someone I could always count on for advice.

I would also like to thank my friends who have helped me in the large undertaking of this thesis. In times where I did not think there were enough hours in the day, you all graciously helped me on multiple occasions. I would not have been able to finish my thesis without you all. Last, but certainly not least, I would like to thank my parents who provided me with constant support and the opportunity to attend graduate school. 


\section{TABLE OF CONTENTS}

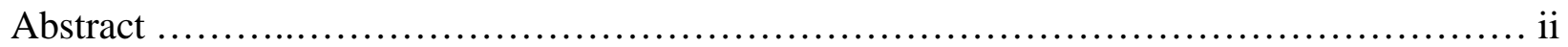

Acknowledgements ............................................................ iii

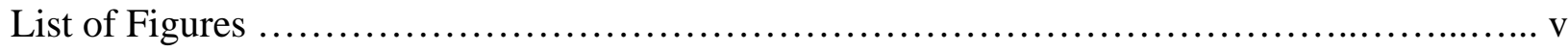

List of Tables ................................................................ vii

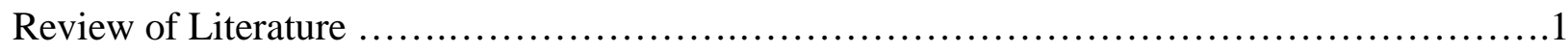

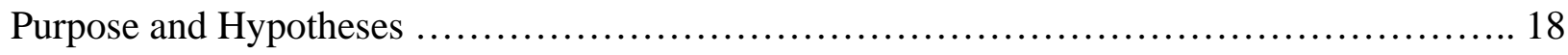

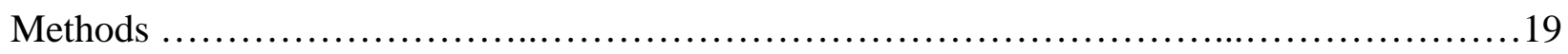

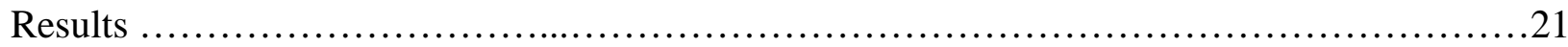

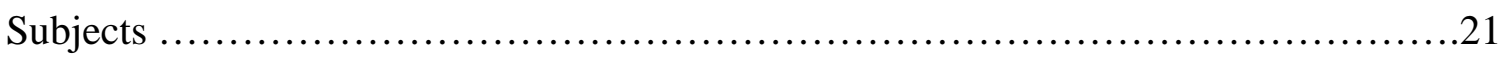

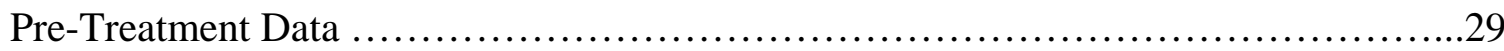

Combined Classes Data (Live vs. Video Presentations) .............................32

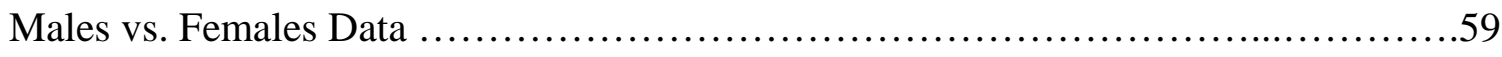

Individual Classes Data (Honors and Regular Classes) ..........................52

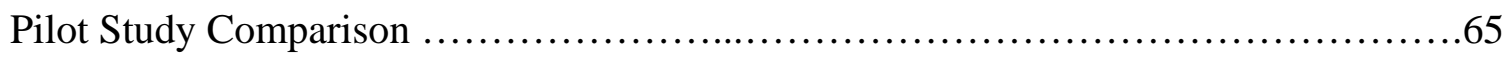

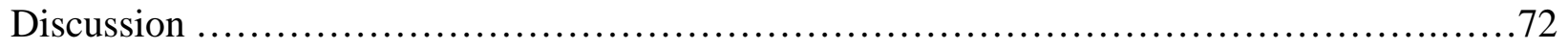

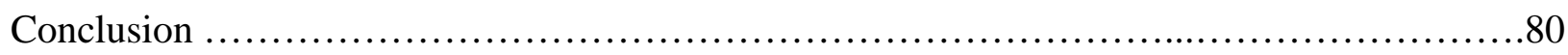

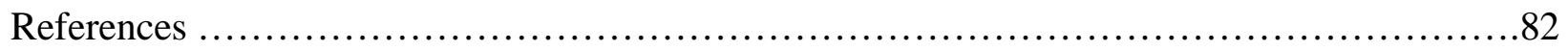

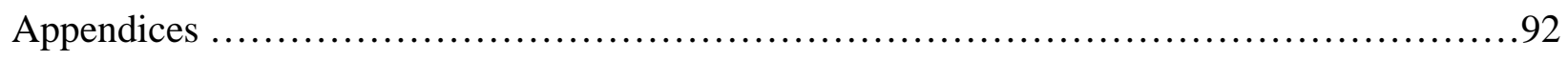




\section{LIST OF FIGURES}

Figure 1. Data comparison diagram: “Stuttering is caused by genetic inheritance” ............25

Figure 2. Data comparison diagram: "People who stutter are shy or fearful” ..................26

Figure 3. Statistically significant differences: honors classes PRE versus

regular classes $\mathrm{PRE}$

Figure 4. Combined classes: statistically significant changes pre-live versus post-live presentations

Figure 5. Combined classes: statistically significant changes pre-video versus post-video presentations.

Figure 6. Combined classes: statistically significant changes post-video versus post-video + live presentations .36

Figure 7. Combined classes: statistically significant changes pre-video versus post-video + live presentations .37

Figure 8. Males: statistically significant changes pre-live versus post-live presentations

Figure 9. Females: statistically significant changes pre-live versus post-live presentations

Figure 10. Females: statistically significant changes pre-video versus post-video presentations

Figure 11. Males: statistically significant changes post-video versus

post-video + live presentations

Figure 12. Females: statistically significant changes post-video versus 
post-video + live presentations

Figure 13. Males: statistically significant changes pre-video versus

post-video + live presentations

Figure 14. Females: statistically significant changes pre-video versus

post-video + live presentations .

Figure 15. Honors class: statistically significant changes pre-live

versus post-live presentation .

Figure 16. Regular class: statistically significant changes pre-live versus post-live presentation .54

Figure 17. Honors class: statistically significant changes pre-video versus post-video presentation .56

Figure 18. Regular class: statistically significant changes pre-video versus post-video presentation .57

Figure 19. Regular class: statistically significant changes post-video versus post-video + live presentations 60

Figure 20. Honors class: statistically significant changes pre-video versus post-video + live presentations .62

Figure 21. Regular class: statistically significant changes pre-video versus post-video + live presentations .63

Figure 22. General items pilot study versus current study comparison 67

Figure 23. Detailed items pilot study versus current study comparison .68

Figure 24. Detailed items pilot study versus current study comparison .69 


\section{LIST OF TABLES}

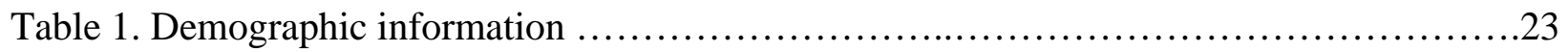

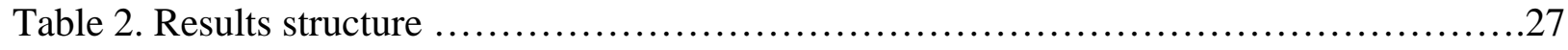

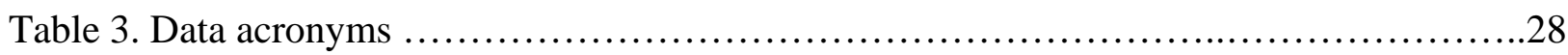

Table 4. Largest and smallest mean differences honors classes PRE

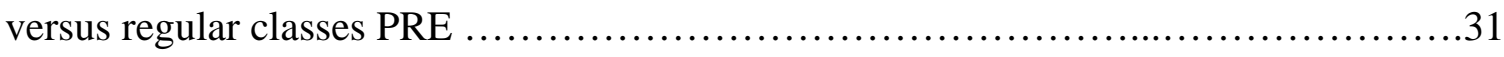

Table 5. Largest and smallest mean differences for combined classes live presentations versus video presentations ...................................... 35

Table 6. Largest and smallest mean differences for combined classes post-video versus post-video + live presentations, pre-video

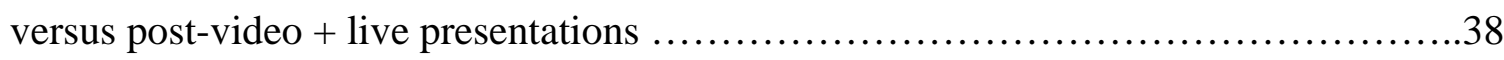

Table 7. Largest and smallest gender mean differences live presentations ........................41

Table 8. Largest and smallest gender mean differences video presentations .....................44

Table 9. Largest and smallest gender mean differences post-video versus

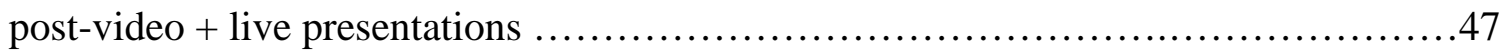

Table 10. Largest and smallest gender mean differences pre-video versus

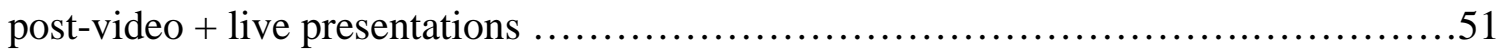

Table 11. Largest and smallest class type mean differences live presentations ...................55

Table 12. Largest and smallest class type mean differences video presentations ..................58

Table 13. Largest and smallest class type mean differences post-video versus post-video + live presentations .............................................. 61

Table 14. Largest and smallest class type mean differences pre-video versus 
post-video + live presentations . ..........................................64

Table 15. Largest and smallest mean differences pilot study versus current study .............70

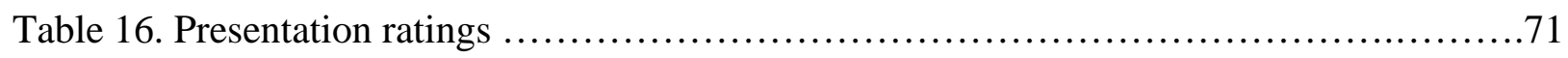

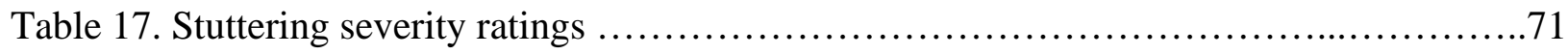

Table 18. Number of participants who thought author's stuttering was faked ...................72

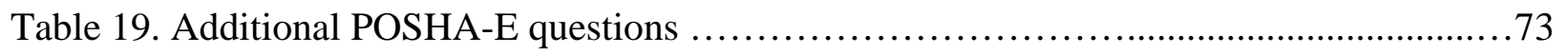




\section{INTRODUCTION}

Extensive research has shown that the public opinion of stuttering is negative (Betz, Blood \& Blood, 2007; Blood, 2000; Blood, Blood, Tellis \& Gabel, 2003; Doody, Kalinowski, \& Armson, 1993; Kalinowski, Stuart, \& Armson, 1996; Craig, Hancock, Tran \& Craig, 2001; D. Shapiro, 1999; Susca \& Healey, 2001; Williams \& Diaz, 1999; Woods \& Williams, 1976). That is, negative stereotypes of persons who stutter have been identified across various study groups. More specifically, negative stereotypes exist among students (Betz, Blood \& Blood, 2007; Evans, Healey, Kawai \& Rowland, 2008; Gabel, Blood, Tellis \& Althouse, 2004; Lees \& Stewart, 2001; Roesti, Tellis, \& Gabel, 2003; St. Louis \& Lass, 1981), teachers (Lass, Ruscello, Schmitt, Pannbacker, Orlando, Dean \& et al., 1992; Lass, Ruscello, Pannbacker, Schmitt, Kiser, Mussa, \& et al, 1994) and even speech-language pathologists (Cooper \& Cooper, 1996; Daly, 1988; Kalinowski, Armson, Stuart, \& Lerman, 1993; Lass, Ruscello, Pannbacker, Schmitt, \& Everly-Myers, 1989; Yairi \& Williams, 1970). The stuttering stereotype even exists for children as young as 3 years old (Betz, Blood \& Blood, 2007). Although stuttering has been shown to reflect negative attitudes, it is believed that public opinion about stuttering can be improved. Most published research also has shown that the public opinion of stuttering is not dependent on exposure to people who stutter (Borsel, Verniers, \& Bouvry, 1999; Craig, Tran, \& Craig, 2003; Doody, Kalinowski, \& Armson, 1993). Nevertheless, there are exceptions (Flynn \& St. Louis, 2007 [see below], Klaasen 2001; Klassen, 2002). If this is the case, then why is the public opinion of stuttering negative? It is for this reason that we must understand where negative public opinion originates. 


\section{Schemas, Stereotypes, and Stigma}

Schemas are used as a means of organization and mental structure. Once a schema has been established, it is difficult to alter even if the schema is an overgeneralization, thus creating a stereotype. Williams and Diaz (1999) agree and add, "Stereotyping can be seen as misclassification of schemas applied to people” (p. 1). The longer a certain schema, or stereotype, is in place, the more difficult it is to change. According to Burgess (2003), stereotypes result from a process of generalizations or assumptions made towards a certain group of people. Stereotypes not only affect the person making a generalization, but also affect the individual, or group of people, toward which the stereotype is directed. It is for this reason that understanding stereotypes is important; that is, their consequences for those being stereotyped can be negative and serious.

In relation to schemas about stuttering, it has been hypothesized that the general public makes observations about their own disfluencies and applies this familiarity to stuttering despite what limited knowledge they have of the subject (White \& Collins, 1984). A recent study (MacKinnon, Hall \& McIntyre, 2007) suggests a hypothesis relating to a theory of anchoringadjustment (Tversky \& Kahneman, 1974). In Mackinnon, Hall and McIntyre’s view, the same stuttering stereotype arises first from anchoring the stereotype in one's own beliefs about normal disfluencies, and then adjusts those beliefs based on one's own experiences with normal disfluencies. Contrary to previous research, ratings of a male stutterer were extremely similar to a male experiencing normal disfluency, both of which differed from ratings of the normal male. The ratings of the male speaker with normal disfluencies were more negative than that of the male stutterer. 
In his seminal work on stigma, Goffman (1963) describes a concept of spoiled identity. A spoiled identity refers to the loss of one's sense of integrity and can lead to a derogatory image of oneself. It also can lead to vulnerability from being labeled negatively or classified without justification. In other words, a spoiled identity identifies the stigma attributed to an individual whereby he or she is viewed as being tainted or discredited in some way. Crocker, Major, and Steele (1998) point out that individuals who are stigmatized come to believe that they possess those negative or unbecoming characteristics that other members society believe they have, and this belief inhibits those stigmatized profoundly. With these assumptions, stigma follows stereotyping, just as stereotyping follows schemas. Goffman (1963) identified three types of stigma: tribal identities (e.g., race, sex, religion, or nation), blemishes of individual character (e.g., disorders, additions, unemployment), and abominations of the body (e.g., physical deformities). In his classification, stuttering would fall under the category of blemishes of individual character. Accordingly, the major impact of stereotypes toward stuttering would not relate to the physical consequences of stuttering, but to its social and psychological consequences (Heatherton, Kleck, Hebl, \& Hull, 2000).

\section{Stuttering: Limitations and Role Entrapment}

Williams and Diaz (1999) and Gabel et al. (2004) concur that stereotyping can lead to stigmatizing the person who stutters. Gabel and his associates point out that people who stutter may face limitations in educational, social, and occupational opportunities due to stereotypes, thus creating multiple stigmas (or stigmata). A limitation in occupational opportunities is often described as role entrapment (Gabel, 2004) which can affect people in educational and social domains. For example, a person who stutters might be viewed as a shy person and therefore 
become trapped in that role. The person might also be viewed as having lower-than-average intelligence, creating role entrapment in educational opportunities. Of course, such beliefs can create self-fulfilling prophecies. If people who stutter feel they are perceived negatively, they tend to act in ways that are consistent with such a view, despite their actual personalities and feelings. Eventually, as noted, they might even begin to believe stereotypes about themselves.

Gabel et al. (2004) surveyed 385 college students regarding their perceptions of career choices for people who either stuttered or did not stutter. The results provided strong evidence that stuttering can lead to role entrapment. The college students rated the hypothetical speakers (on a scale from 1-5) for various career choices, wherein higher mean scores indicated more suitable careers. Respondents gave people who stutter a mean score of 3.83, but people who do not stutter, a mean score of 4.23. More specifically, people who stutter were scored the lowest for the job titles of attorneys (2.71), judges (2.95), and speech-language pathologists (3.02). In contrast, people who did not stutter were scored 4.36, 4.39, and 4.26, respectively, for these professions. The highest score attributed to people who stutter was the role of a computer programmer (4.42), and the highest score for people who do not stutter was the role of a physician (4.43). People who stutter scored lower than people who did not stutter in all roles, except for computer programmer.

Gabel et al. (2004) further measured the occurrence of role entrapment by comparing differences in college students' perceptions of career advice for people who stutter and people who do not stutter. The results showed that people who stutter were less likely to be advised to pursue 20 of the 43 careers listed on the questionnaire. On all 20 careers, the mean response for people who stutter was less than 4.00 , the cutoff score indicating that participants, as a group, did not agree that a person who stutters should pursue a given career. The results of this study clearly 
suggest that people who stutter are victims to role entrapment and, hence, stigma in the workplace.

In a study by DeLoach (1989), employers reported being cautious towards placing individuals with disabilities into management or leading positions. This was true for people with physical, mental, emotional, or communication disabilities. Rice and Kroll (1997) found that of 568 adults who stutter who were surveyed, $70 \%$ of them believed they could have had better jobs if they did not stutter, and 56\% had chosen a job that required less speaking than other potential or desirable jobs. Another 35\% reported that they believed (a) that stuttering had hindered their chances of promotions, (b) that they had experienced discrimination, or (c) that their supervisors had the wrong impression about their job competence because they stuttered.

\section{Stuttering: Misinformation}

Why do stuttering stereotypes exist so consistently across different populations? One explanation is that misinformation constantly circulates throughout the population. For example, if a curious person were to visit "www.dictionary.com" and search for the definition of "stuttering," the website would reveal the following: "a disorder of vocal communication marked by involuntary disruption or blocking of speech (as by spasmodic repetition or prolongation of vocal sounds), by fear and anxiety, and by a struggle to avoid speech errors.” This website receives its definitions and information from third parties, in the case of stuttering, from a medical dictionary (“Stuttering”, n.d.). Yet the definition for stuttering is not still entirely accurate. While stuttering can be described as involuntary disruption or blocking of speech, stuttering is not caused by fear, anxiety or by struggling to avoid speech errors (e.g., Guitar, 2006). It is impossible to know how many people use sources such as "dictionary.com” to search 
for information, but it can be assumed that the number is likely large. Further, it can be surmised that no one would question the definition because it originates from a medical dictionary. Misinformation like this would only further solidify someone's stereotypes, or create new stereotypes with what is supposed to be factual information.

D. Shapiro (1999) explains how such misinformation begets more misinformation. In most cases when parents bring their stuttering children to a physician's office, they typically are told, “Don’t worry about it. He will outgrow it.” While it is true that most children who stutter will outgrow their stuttering, at least 20\% will not (D. Shapiro, 1999). Other misinformation about stuttering can also have negative consequences for those who stutter. For example, in a survey conducted among university students, Nichols (1987) found that 30\% of her participants thought that stuttering was a sexually transmitted disease. In this example, the problem of misinformation begetting misinformation is dramatic.

\section{Stuttering: Public Opinion Among Speech-Language Pathologists}

Daly (1988) writes that when clinicians focus on negative aspects of stuttering instead of the positive goals of a therapy session, clients can become discouraged. He hypothesized that this can be the cause of high failure and dropout rates among stuttering clients. Daly goes on to point out that "The clinician's attitudes toward stuttering and people who stutter have as much to do with the successful treatment of this disorder as the methods selected for therapy” (p. 34).

Lass et al.'s (1989) findings were congruent with the idea that speech-language pathologists (SLPs) hold many stereotypes about people who stutter. Eighty-one SLPs were asked to list as many adjectives of people who stutter as they could think of about four hypothetical people who stutter: (a) an adult male who stutters (b) an adult female who stutters 
(c) an 8-year-old male who stutters, and (d) an 8-year-old female who stutters. The authors highlighted three important findings from 527 traits listed by the participants: (a) more adjectives were associated with males who stutter than females, regardless of age; (b) extremely similar adjectives were associated with both groups of males who stutter and females who stutter; and (c) a large majority of adjectives associated with people who stutter, both male and female, were negative. Of all the adjectives listed, $70 \%$ were judged to be negative, $24 \%$ were positive, and $6 \%$ were neutral. The study also showed that $93 \%$ of the adjectives described personality, while the rest pertained to physical appearance (3\%) or mental abilities (4\%). It is also important to note that $88 \%$ of all adjectives listed were identical for the four different hypothetical persons who stutter.

Another study by Kalinowski and Armson (1993) showed similar results using a Likert scale adapted from Woods and Williams (1979). In the 1993 study, 58 speech-language pathologists and 137 members of the general public completed a 25 -item semantic differential scale. Concurring with the results of Lass et al. (1989), clinicians' attitudes towards people who stutter were negative. Clinicians assigned adjectives such as "guarded,” "nervous,” “tense,” “reticent," "insecure,” and "hesitant.” Surprisingly, despite speech-language pathologists' 10+ years of experience with people who stutter, there were no significant differences between their attitudes and those of the general public. On the other hand, using an experimental version of the Public Opinion Survey of Human Attributes (POSHA-E) (explained below), experienced SLPs' attitudes were often more positive than those of college students from other majors (St. Louis, Tellis, Tuanquin, Wolfenden \& Nicholson, 2004). Moreover, for some POSHA-E items, attitudes of SLPs who, further, were Board Recognized Specialists in Fluency Disorders (Specialty Board on Fluency Disorders, 2009), were even more positive. 
It can be hypothesized that while teaching speech-language pathology students about stuttering, stereotypes may be reinforced (e.g., St. Louis \& Lass, 1981). We might assume that students are taught what people who stutter may or may not feel. For example, it is often written that a person who stutters might feel fearful, shy, angry, or embarrassed about their stuttering (D. Shapiro, 1999). Therefore, future SLPs are likely to acquire predisposed assumptions about people who stutter, thus reinforcing a stereotype - even though stutterers may not feel this way at all. This phenomenon of reinforcing stereotypes can go beyond the classroom into actual clinical practice. Yairi and Williams (1970) wrote, “A speech clinician who has grown up in an environment in which stutterers were considered 'nervous' would probably expect nervous behavior from a stutterer. Stutterers, as well as nonstutterers, may at times manifest behavior which the particular clinician evaluates as 'nervousness'. The clinician may thus find ‘confirmation’ of his expectation” (p. 168).

\section{Adolescent Opinions About Stuttering}

As stated previously, abundant research has documented a negative stuttering stereotype among diverse populations. Yet, there have been only a few studies conducted with adolescents. McGee et al. (1996) conducted a study in which the effects of viewing a documentary videotape were measured. After watching the video, it was reported that participants held a stronger negative stereotype towards people who stutter. Kirsch (2007) conducted a study in which adolescents were randomly assigned to specific groups to view one of two videotapes where a person who stutters is interviewing for a job. Respondents were 251 adolescents, 130 girls and 121 boys in the $8^{\text {th }}$ or $11^{\text {th }}$ grades, with an average age of $16 \frac{1 / 2}{2}$ years. One videotape showed an adolescent girl with fluent speech; the other showed a different girl who stuttered. After 
participants viewed one of the two videotapes, they completed a survey where they rated the speaker on seven personality traits using bi-polar adjective pairs on a 5-point semantic differential scale. The adjective pairs were: (a) Friendly - Unfriendly, (b) Good sense of humor Poor sense of humor, (c) Outgoing - Shy, (d) Confident - Unsure, (e) Relaxed - Tense, (f) Understandable - Not understandable, (g) Competent - Incompetent. After viewing the videotape of an adolescent girl stuttering, participants were asked to complete 10 additional survey questions exploring perceptions of different social issues.

Kirsch reported that for both the $8^{\text {th }}$ and $11^{\text {th }}$ grades, the mean ratings for the speaker who stuttered were more negative than the mean ratings of the speaker who did not stutter. Further, for the person who stuttered, the mean ratings for the bi-polar adjective pairs of "sense of humor," "outgoing," "confident," and "relaxed” were less than the 2.5 neutral value. By contrast, only the "sense of humor" adjective pair obtained a mean rating less than the neutral value for the speaker who did not stutter. It was also reported that females in both the $8^{\text {th }}$ and $11^{\text {th }}$ grades were generally more positive than the males, but the gender difference was not statistically significant.

For each of the seven adjective pairs, participants were asked to explain why they selected their rating points. Each explanation was assigned a negative or positive value. It was noted that the negative responses outnumbered the positive responses for the person who stuttered for both $8^{\text {th }}$ and $11^{\text {th }}$ graders. Fifty four percent of $8^{\text {th }}$ grader responses were negative for a PWS (person who stutters) compared to 21\% for a PWNS (person who does not stutter), and 16\% were positive for a PWS contrasting 46\% for PWNS. Eleventh graders showed less difference in that $46 \%$ of comments for a PWS were negative as to $31 \%$ for a PWNS. Also, $22 \%$ were positive for a PWS and 38\% for a PWNS. 
Kirsch concluded that there were statistically significant negative attitudes towards people who stutter but that gender differences were not significant. Further, he suggested that while the mean ratings for "friendliness," "understandability," and "competence” were lower than those of the person who did not stutter, they were above the neutral value of 2.5, suggesting that adolescents do not necessarily have a negative stereotype towards people who stutter. This conclusion is odd, given the above findings however, especially since a "neutral" attitude has yet to be reliably documented on judgments using adjective pairs.

\section{Males and Females Attitudes Towards Stuttering}

Similar to the limited research on adolescent's attitudes towards stuttering, little research has been conducted studying gender differences (Evans et al., 2008; Hartford \& Leahy, 2007; Langevin \& Hagler, 2004; Patterson \& Pring 1991; Weisel \& Spektor, 1998). Most studies concur with Kirsch (2007) in that there are no gender differences in attitudes towards stuttering (Evans et al., 2008; Hartford \& Leahy, 2007; Langevin \& Hagler, 2004; Patterson \& Pring 1991). Although, a few studies (Van Borsel, Verniers \& Bourvry, 1999; Weisel \& Spektor, 1998) found that females show more positive attitudes towards PWS.

\section{Changing Public Opinion}

While there has been a great deal of research measuring public opinion of stuttering and its effects, it must not go unnoticed that there has been very little research on how to change public opinion. Only four studies were located that attempted to do so (Leahy, 1994; McGee et al., 1996; Reichel \& St. Louis, 2004; Snyder, 2001). Three of the four studied graduate-level speech-language pathology students, while the fourth (McGee et al., 1996) focused on 
adolescents. One of the four documented positive changes (Reichel \& St. Louis, 2004), one documented little or no positive changes in public opinion (Snyder, 2001), and two actually recorded a negative change of attitudes towards stuttering (Leahy, 1994; McGee et al., 1996).

The most recent published study was Reichel and St. Louis (2004), also included in St. Louis, Reichel, Yaruss \& Lubker (in press), who surveyed 77 students in graduate level courses in fluency disorders from two universities. Students completed three different questionnaires at the beginning and end of the semester. The first questionnaire was the Emotional Intelligence Scale (EIS), which is a self reported instrument measuring the ability to identify and express regular emotions in oneself and in others (Schutte \& Malouff, 1999). Its purpose was to measure such constructs as optimism, impulse control, and strong attention to feelings, mood repair, and empathy. The second questionnaire was the experimental edition of the Public Opinion Questionnaire of Human Attributes (POSHA-E) (St. Louis, 2005; St. Louis, Lubker, Yaruss, Adkins \& Pill, 2008), a questionnaire under development that measures knowledge, beliefs, reactions, feelings, and comparative attitude toward stuttering along with several other human conditions. The third was the Bipolar Adjective Scale (Woods \& Williams, 1976), consisting of 25-paired adjectives and was designed to describe perceived personality attributes of people who stutter.

Reichel \& St. Louis reported 10 statistically significant changes before and after the fluency courses, all of which were interpreted as positive changes in attitudes. Some of these items included, "My overall impression of a person who has a stuttering disorder," "The amount I know about people who have stuttering disorders," "I believe stuttering is caused by genetic inheritance" and "If I were talking to a person who stutters, I would feel frustrated." Nevertheless, there were several nonsignificant negative changes as well. Some of these items 
were, "I believe stuttering is caused by psychological factors," "I believe stuttering is caused by learning or habits," "I believe stuttering is caused by imitating other people" and "I believe stuttering is caused by a virus or disease," all of which showed moderate negative changes. This highlights an important possibility supporting the potential of public awareness as a change agent that is contrary to Burgess's (2003) assertion that education does not dramatically change public opinion.

Another study by Snyder (2001) showed similar results. As in Reichel and St. Louis’s (2003) study, participants were graduate students who were enrolled in a fluency class. The participants completed the Clinicians Attitudes Toward Stuttering (CATS) inventory (Cooper, 1975) before and after watching two short films. The first film was taken from Speaking of Courage while the second one was Effects of Altered Auditory Feedback at Fast and Normal Speaking Rates. The first film was shown to evoke emotional responses from the participants, while the second film was shown to evoke factual responses. The purpose was to determine if either type of video was capable of changing attitudes in a positive manner. Each video was adjusted to be approximately the same length.

The results of this study resulting from watching Speaking of Courage (Bondarenko, 1992a) were subtle (Snyder, 2001). Significant change occurred for only one CATS question, “Operant programs for stutterers have been found to be effective.” The pre and post responses to this question changed from a median response of "undecided" to "moderately agree.” The second video, Effects of Altered Auditory Feedback on Stuttering Frequency at Normal and Fast Speaking Rates, showed more promising results. Significant changes were seen for three questions: "Chances are that most stuttering is the result of multiple coexisting factors," "There is no such thing as a 'primary stutterer' (a stutterer who stutters but isn't aware of it),” and 
"Stuttering behaviors are relatively easy to modify." Snyder stated that his study showed perceptual changes regarding stuttering are possible, but not frequent or intense enough to show any authentic change. He also states that it could be argued that the differences that were found were related to the measurement instrument's reliability and were not reliable changes at all.

Leahy (1994) reported similar negative results to changing clinician's attitudes towards stuttering. Seventeen students in a local university were surveyed over a one-year span in an attempt to change their attitudes. Seven of the students were each assigned to work with a stuttering client. Five of the students attended individual therapy and group therapy with their clients; the remaining two attended only individual therapy. Group therapy consisted of discussions about stuttering while individual therapy taught specific speech fluency techniques. The remaining students were only involved in the class and had no direct contact with people who stutter; however, during the class, each student was asked to attempt pseudo-stuttering for a day. Pseudo-stuttering was assigned so that students could experience the reactions to stuttering first hand.

Leahy adapted and shortened the Woods and Williams (1976) scale to an 11-item version, e.g. calm versus nervous, unfriendly versus friendly, and so on. After one year, she found that participants who attended the group therapy sessions had more positive outlooks towards people who stutter on certain items. They regarded people who stutter in a more positive light relative to pleasantness, quietness, and extroversion. However, attitudes changed in a negative direction for nervousness, tension, and reticence. It should be noted that the attributes of nervousness, tension, and reticence were the stereotypes in question for this study; therefore, Leahy (1994) concluded from her results that the group and individual therapy changed attitudes negatively. It should be noted that only 13 of the 17 students agreed to complete the rating scales 
to measure attitude changes, a problem recognized by Leahy that could lead to difficulties in generalizing the results.

McGee’s et al. (1996) study is different from the other three studies in that it dealt with normally speaking adolescents instead of graduate students in speech-language pathology. It also used the entire Woods and Williams (1976) 25-item seven-point Bipolar Adjective Scale. Participants in the study viewed a videotape called Voices to Remember (Bondarenko, 1992b), a one-hour dramatic presentation of the stories of a number of adults who stutter, all with positive outcomes, and completed the questionnaire before and after watching the videotape.

Surprisingly, after watching the video, what were already negative attitudes towards stuttering, became more negative. Specifically, a hypothetical male who stutters was rated as being more guarded, nervous, shy, tense, withdrawn, quiet, reticent, avoiding, afraid, hesitant, and insecure after viewing the tape. In three of these 11 adjectives, i.e., being more withdrawn, reticent, and fearful, the differences were statistically significant. McGee, Kalinowski, and Stuart (1996) noted that stereotypes exist in adolescents and hypothesized that the videotape reinforced stereotypes rather than changed them.

\section{PILOT STUDY}

In 2006, a pilot study (Flynn, 2007; Flynn \& St. Louis, 2007) was conducted as follows. An adaptation of the POSHA-E was distributed to two classes in a local high school to achieve a baseline measure for public opinion of stuttering in adolescents. One was an honors health class consisting of 16 students containing 10 sophomores, five freshman, and one junior. The other was a regular health class consisting of 23 students and had 17 sophomores, five freshmen, and one junior. The next day, the author, a person who stutters, spoke to these two classes about his 
stuttering. During the talks, according to his own appraisal, his stuttering ranged from moderate to severe. After the presentation, an identical questionnaire was given to the students to measure any changes in attitudes. Results from the pre-test indicated that adolescents had negative stereotypes towards people who stutter. Comparing all students combined for both classes using multiple t-tests with the Bonferroni correction $(\mathrm{p}<0.005)$ (rationale presented in the Results section below), there were eight statistically significant positive attitude changes towards stuttering. Further analysis considered percentage changes in ratings for each question. For example, on the item, "People who stutter are nervous or excitable,” $6.1 \%$ of participants marked a higher score on their post-questionnaires, $75.8 \%$ marked a lower score, and $18.2 \%$ marked the same score. A change from pre to post of $50 \%$ or more was arbitrarily deemed as a noteworthy trend. In this analysis, there were 21 percentage changes $(\mathrm{p}>50 \%)$ showing noteworthy trends for positive attitude change.

Altogether, the data showed statistically significant positive mean changes in the following questions: “The amount I know about people who stutter," "My knowledge of stuttering comes from...my experience with people who stutter," "I believe stuttering is caused by...nervousness," "I believe stuttering is caused by being mentally/emotionally abuse," "I believe stuttering is caused by being physically abused", "If I were talking to a person who stutters, I would feel curious to know more about stuttering," "People who stutter are nervous or excitable," and "Stuttering is a disability." The largest change was for the question, "People who stutter are nervous or excitable" (5.00 vs. 3.22 on a 1-9 scale), with a t-test score of 0.00001 . Within the 21 noteworthy trends, the three largest percentage changes were for: "People who stutter are nervous or excitable” (75.8\% lower, 6.1\% higher, 18.2\% unchanged), “I believe stuttering is caused by nervousness" (75.0\% lower, $16.7 \%$ higher, $8.3 \%$ unchanged), and "I 
believe stuttering is caused by being mentally/emotionally abused” (71.0\% lower, $12.9 \%$ higher, $16.1 \%$ lower).

Data for honors and regular classes showed considerable differences. Regular class students' ratings did not change significantly for any POSHA-E items, while those in the honors class showed statistically significant changes in all of the same items listed above in the combined classes' data, except for, "Stuttering is a disability.” The item, "I believe stuttering is caused by nervousness" (6.23 vs. 3.14), showed the largest change. While participants within the regular class showed no statistically significant attitude changes, 20 percentage changes were deemed noteworthy. The three largest percentage changes were for questions, "People who stutter are nervous or excitable” (76.9\% lower, 7.7\% higher, 15.4\% unchanged), "People who stutter are shy or fearful” (69.2\% lower, 23.1\% higher, 7.7\% unchanged), and "I believe stuttering is caused by nervousness” (66.7\% lower, $26.7 \%$ higher, $6.7 \%$ unchanged). Honors class participants PRE versus POST ratings reflected 27 noteworthy trends. The three largest percentage changes were for the questions, "I believe stuttering is caused by being mentally/emotionally abused” (83.3\% lower, 5.6\% higher, 11.1\% unchanged), “I believe stuttering is caused by nervousness" (81.0\% lower, 9.5\% higher, 9.5\% unchanged), and "I believe stuttering is caused by psychological factors” (78.9\% lower, 15.8\% higher, 5.3\% unchanged). These less conservative trend comparisons, together with the statistically significant differences, suggest the potential to alter adolescent opinions' about stuttering in a positive direction.

After participants heard the oral presentation, they were asked to write comments about the presentation and to rate it on a scale of 1 to 9 . The overall rating of the presentation was 8.19 
(7.88 for the regular class and 8.41 for the honors class). Almost all of the comments were complimentary. Some of the remarks were:

I really learned a lot from this presentation. It made me want to know more about stuttering. Mr. Flynn is a very nice person and I feel for him very much. He is just like other people \& because of him I will never laugh at a person who stutters. I now think higher of people who have a stutter. I have more respect for them than I do for people who don't stutter because they really have been through a lot and I think they deserve more respect. Overall, this presentation was very interesting and it taught me a lot. Thank you very much for coming to speak to us.

I learned a lot about stuttering and it was an eye opener for me. I totally respect Tim for everything he does. Also, for how confident and positive he is. I don't think I would be as comfortable with talking as he was.

These evaluative comments included a surprising and unusual finding, i.e., that the stuttering was not real. Sample comments included:

It was a good speech. I wasn't sure if it was an example of stuttering or real. Thank you for giving us information on stuttering. Do you really stutter? This presentation was pretty cool. At first I thought that he was just pretending to stutter, but I'm not so sure anymore...

After reading these responses, the author tallied the number of students who thought that his stuttering was real, or merely a fake example of stuttering. Forty of 70 students (57\%) thought the author's stuttering was fake. 
The pilot study provided the primary background for formulating the current study. It was noted that further research in this area should be conducted to test different presentation modes for attempting to change attitudes of high school students.

\section{PURPOSE AND HYPOTHESES}

The purpose of the proposed study was twofold: (a) to measure the extent of positive change in the attitudes of adolescents toward stuttering, and (b) to compare two different methods of changing attitudes. Specifically, the study determined the effects on measured attitudes of:

(a) Presentations to high school classes by the author designed to improve attitudes towards stuttering.

(b) A live presentation versus a video presentation recorded for a "True Life" segment for MTV that featured the author.

The following research hypotheses were tested:

(a) High school students will show evidence of the stuttering stereotype.

(b) A live presentation will have a greater positive impact on participants than a video presentation.

(c) A shortened live presentation following the video presentation will further produce a positive attitude change.

(d) High school students in honors classes will have more positive attitudes towards stuttering than students in regular classes and will be more likely to change their attitudes positively.

(e) Male and female students will have equivalent attitudes towards stuttering. 


\section{METHOD}

The questionnaire for this research study was adapted from the POSHA-E. The POSHA-E was chosen for reasons mentioned earlier. It was designed for use with subjects from later childhood through adulthood, it contains a minimum of slang terms and terminology and the layout of the questionnaire is simple and straightforward. Most importantly, reliability and validity have been documented for the questionnaire (St. Louis, Lubker, Yaruss, Adkins \& Pill, 2008; St. Louis, Lubker, Yaruss \& Aliveto, in press; St. Louis, Reichel, Yaruss \& Lubker, in press)

The adaptation of the POSHA-E questionnaire used in this study had the following sections: (a) a general section comparing stuttering to other human attributes, (b) a detailed section of opinions about stuttering and reactions to people who stutter, and (c) a short section on biographical information (See Appendix 12). The general section provided a basis for comparison of stuttering with three other attributes: a negative attribute, “overweight,” a neutral attribute, "left-handed,” and a positive attribute, “intelligent.” The detailed stuttering section asked questions about causes of stuttering, characteristics of people who stutter, social interactions with people who stutter, and reactions to a person who stutters (e.g., anger, embarrassment, or nervousness). The biographical section requested participants’ age, sex, and class ranking. Changes from the previous version of the POSHA-E included: changing from a 9point scale to a 5-point scale for the general section, changing from a 9-point scale to a "Yes," “No,” or "Not Sure” selection format on the detailed stuttering sections, and a questionnaire shortening or condensing of specific questions. All changes were made to create a more efficient and easier to understand questionnaire. Also, for this study, three new questions were added to the questionnaire: "Have you heard the presenter speak to you before?" "Have you seen the 
documentary video entitled, True Life: I Stutter before (for the video condition only) and "Do you think the presenter's stuttering was fake during the presentation or on the video?”

The study was conducted at a local high school in two separate health classes. A health teacher agreed to allow the author to conduct research in her classes. The teacher first asked students to take consent forms home, read about the research, and consider signing them with their parents. Those who opted to participate in the study by signing consent forms were divided into two treatment groups. Both groups completed a POSHA-E questionnaire 4-5 days before they were exposed to the presentation about stuttering. The first group listened to a 45-minute live oral presentation by the author directly after the questionnaire was completed. As soon as the presentation was completed, participants completed the same questionnaire. The second group watched a 45 minute video entitled, True Life: I Stutter (Schneider, 2007) (see below). After watching the video, the second group of participants completed the same questionnaire. Immediately after completing the post-questionnaire, the author spoke to them. After giving a shorter 20-minute-version of the oral presentation, the second group of participants completed a third questionnaire to test the further alteration of attitudes. In total, the first group of participants completed the adapted POSHA-E before and after hearing a live presentation. The second group of participants completed the same POSHA-E before and after watching the 45 minute video, and again, after listening to a shortened version of the live presentation. After both the live and video presentations were completed, participants in both groups were asked to write journal responses to the presentations.

The 45-minute oral presentation covered four basic areas: factual information about stuttering, the author's personal experiences and/or stories about stuttering, some of his coping mechanisms for stuttering, and his personal insights and beliefs towards stuttering. The 
presentation was similar to the one from the pilot study. This presentation, however, was more structured. Each subsection targeted different aspects of attitude towards stuttering. For example, factual information about stuttering included a more detailed description of causation of stuttering. Some journal remarks from the pilot study included: "Could go into more detail about the problem and give more info on what causes it." Another comment was, "He was nice and funny. I would like to meet more people who stutter. He could have said a few more things about why stuttering happens though.” Other journal remarks were analyzed to create a more detailed and effective presentation to change attitudes.

The 45-minute video entitled, True Life: I Stutter (Schneider, 2007) is a recent addition to the MTV network’s documentary series. Each episode presents a particular topic, in this case, stuttering. The video follows the lives of the author, a 23-year-old overt male stutterer in graduate school, as well as two female stutterers, a 20-year-old covert stutterer attending an intensive fluency shaping program and a 20-year-old overt stutterer who was trying to become Miss New York in a beauty pageant. This video was chosen because (a) it is a recent and public documentary about stuttering (b) it costars the author, and (c) the series, True Life, is aimed towards adolescents, the target population for this study.

\section{RESULTS}

\section{Subjects}

A total of 83 students participated in this study. Thirty-eight were students in two honors classes (20 and 18, respectively), and 45 were members of two regular classes (20 and 25, respectively). Of the 83 total participants, 33 were male (39.8\%) and 50 were female (60.2\%). Within the honors classes, $23.7 \%$ were males and $76.3 \%$ were females. Within the regular classes, $53.3 \%$ were males and $46.6 \%$ were females. Participants within the honors classes were 
older, with an average age of 16.9 years, whereas the regular classes had an average age of 15.8 years. Honors classes had $10.5 \%$ seniors, $34.2 \%$ juniors, $47.4 \%$ sophomores, and $7.9 \%$ freshmen, whereas regular classes had $73.3 \%$ sophomores and $26.7 \%$ freshmen. A large majority of participants were Caucasian (86.7\%), whereas 2.4\% were African American, 4.8\% were Asian, 2.4\% were bi-racial, and 3.6\% were other ethnic backgrounds. Other demographical information is provided in Table 1.

Appendix 1 shows participants' experience with five attributes, i.e. obesity, left handedness, stuttering, mental illness, and intelligence. With regard to stuttering, $21.2 \%$ of males and 32\% of females did not know a person who stutters (PWS), 51.5\% of males and 34\% of females had an acquaintance who was a PWS, 30.3\% of males and 30\% of females had a close friend or relative who stutters, and no participants stuttered themselves. 
Table 1

Demographic information

\begin{tabular}{|c|c|c|c|c|c|c|c|c|}
\hline & \multicolumn{2}{|c|}{$\begin{array}{c}\text { Honors Class } \\
\text { Live } \\
\text { Presentation }\end{array}$} & \multicolumn{2}{|c|}{$\begin{array}{l}\text { Honors Class } \\
\text { Video } \\
\text { Presentation }\end{array}$} & \multicolumn{2}{|c|}{$\begin{array}{c}\text { Regular Class } \\
\text { Live } \\
\text { Presentation }\end{array}$} & \multicolumn{2}{|c|}{$\begin{array}{c}\text { Regular Class } \\
\text { Video } \\
\text { Presentation }\end{array}$} \\
\hline & Males & Females & Males & Females & Males & Females & Males & Females \\
\hline Sample Size & 5 & 15 & 4 & 14 & 9 & 11 & 15 & 10 \\
\hline \multicolumn{9}{|l|}{ Native } \\
\hline English & 4 & 15 & 4 & 13 & 8 & 11 & 14 & 9 \\
\hline Spanish & 0 & 0 & 0 & 0 & 0 & 0 & 1 & 1 \\
\hline French & 0 & 0 & 0 & 0 & 0 & 0 & 0 & 0 \\
\hline Other & 1 & 0 & 0 & 1 & 1 & 0 & 1 & 0 \\
\hline \multicolumn{9}{|l|}{ Race } \\
\hline Caucasian & 4 & 11 & 4 & 13 & 7 & 11 & 13 & 9 \\
\hline \multicolumn{9}{|l|}{ African } \\
\hline American & 1 & 1 & 0 & 0 & 0 & 0 & 0 & 0 \\
\hline Asian & 0 & 1 & 0 & 1 & 1 & 0 & 1 & 0 \\
\hline Bi-Racial & 0 & 2 & 0 & 0 & 0 & 0 & 0 & 0 \\
\hline Other & 0 & 0 & 0 & 0 & 1 & 0 & 1 & 1 \\
\hline \multicolumn{9}{|l|}{ Age (yr) } \\
\hline 15 & 0 & 0 & 0 & 0 & 6 & 4 & 6 & 1 \\
\hline 16 & 1 & 5 & 5 & 4 & 13 & 7 & 18 & 9 \\
\hline 17 & 3 & 6 & 13 & 10 & 1 & 0 & 1 & 0 \\
\hline 18 & 1 & 4 & 0 & 0 & 0 & 0 & 0 & 0 \\
\hline Mean Age (yr) & 17.0 & 16.9 & 16.8 & 16.7 & 15.7 & 15.8 & 15.8 & 15.8 \\
\hline \multicolumn{9}{|l|}{ Class Rank } \\
\hline Freshman & 0 & 3 & 0 & 0 & 3 & 4 & 4 & 1 \\
\hline Sophomore & 0 & 8 & 1 & 9 & 6 & 7 & 11 & 9 \\
\hline Junior & 2 & 4 & 2 & 5 & 0 & 0 & 0 & 0 \\
\hline Senior & 3 & 0 & 1 & 0 & 0 & 0 & 0 & 0 \\
\hline
\end{tabular}

Structure and Logic of the Results Section

This study compared five groups of participants: (a) pre-treatments versus pre-treatments (b) live presentations versus video presentations (regular and honors classes combined), (c) males versus females (d) honors versus regular classes, and (e) pilot study versus current study. Within (a) pre-treatments versus pre-treatments, mean scores were compared before participants heard either the live presentation or the video presentation. These data would determine whether 
or not high school students demonstrated evidence of negative attitudes or stereotypes toward stuttering before any treatment. These data were compared for (b) live presentations versus video presentations, (c) males versus females, (d) honors versus regular classes, and (e) the pilot study. Within (b) live versus video presentations, this study also compared four sampling points: pre-live versus post-live, pre-video versus post-video, post-video versus post-video + live presentations and pre-video versus post-video + live presentations. Post-live and post-video comparisons refer to questionnaire item mean scores after the live or video presentation was presented. This relates to the primary purpose of the study, i.e., to determine whether or not a live presentation elicited a greater change in attitudes than a video presentation. Pre-live versus pre-video were analyzed to measure any significant differences in pre-means between the live and video presentations, simply as a control to determine the extent to which the randomly assigned treatment were given to participants with similar versus dissimilar respondent groups. Post-video versus post-video + live, in the video group only, refers to mean scores after the 45minute video presentation versus the 20-minute shortened live presentation that followed the video. This comparison is needed to examine whether or not the 20 -minute shortened live presentation had any further effect on participants who had already watched the video presentation. Next, pre-video versus post-video + live comparisons were analyzed, i.e., pre-video questionnaire items mean scores were compared to mean scores following both the video presentation and the 20-minute shortened live presentation. At first inspection, this analysis may seem unnecessary and redundant, but such comparisons provide useful information because certain questionnaire items changed direction from pre-video versus post-video, and post-video versus post-video + live. This can be seen in Figure 1 for questionnaire item, "I believe stuttering 
is caused by genetic inheritance” (35 [pre-video] vs. -12 [post-video] vs. 74 [post-video + live]) whereas a change of direction in mean scores is noted from pre-video versus post-video + live.

Figure 1. Stuttering is caused by genetic inheritance

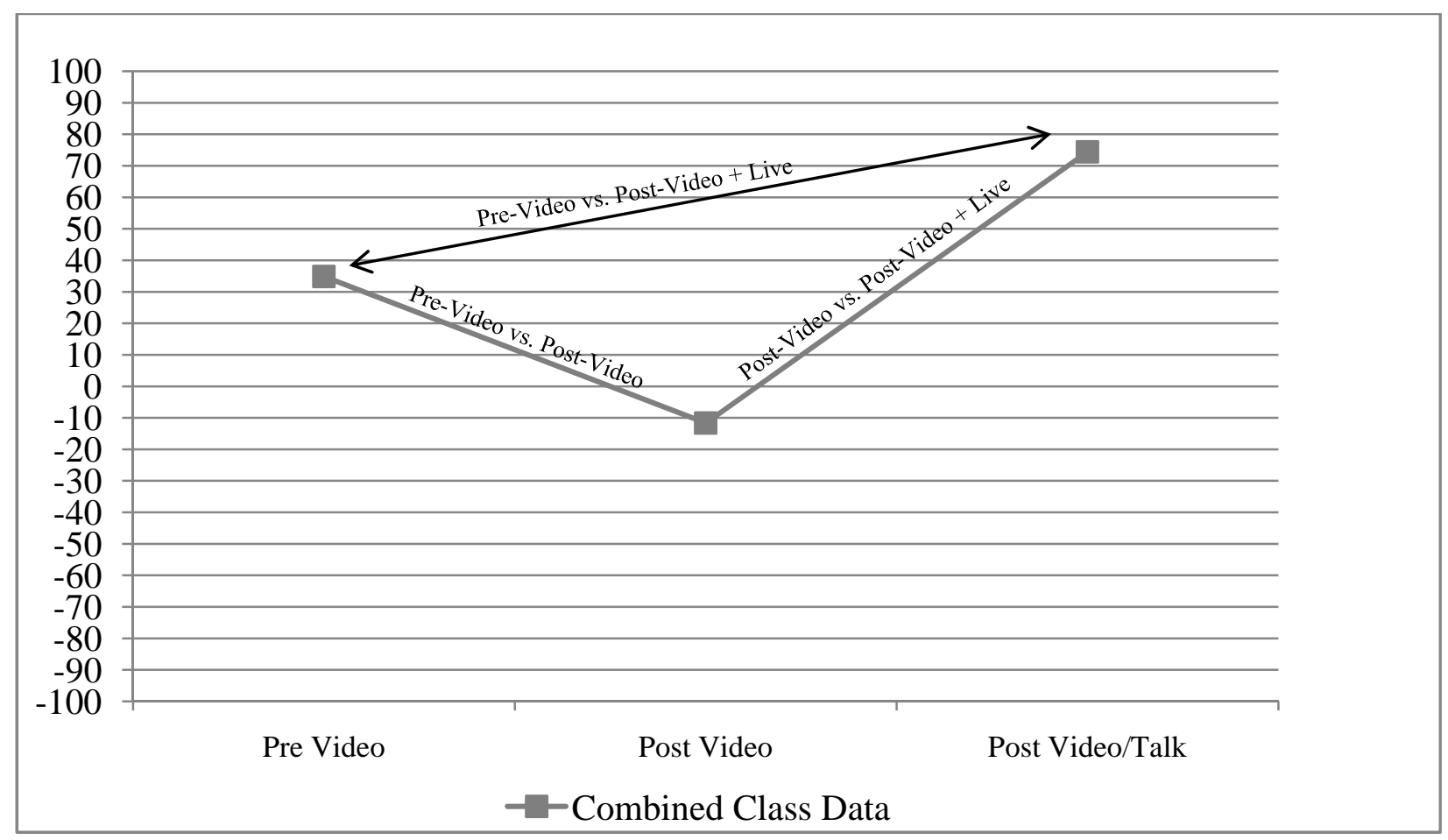

Also, certain questionnaire items were not statistically significant from pre-video versus postvideo but were statistically significant from pre-video versus post-video + live. This can be seen in Figure 2 for questionnaire item, "People who stutter are shy or fearful” (21 [pre-video] vs. 9 [post-video] vs. -28* [post-video + live) (* indicates statistical significance). 
Figure 2. People who stutter are shy or fearful

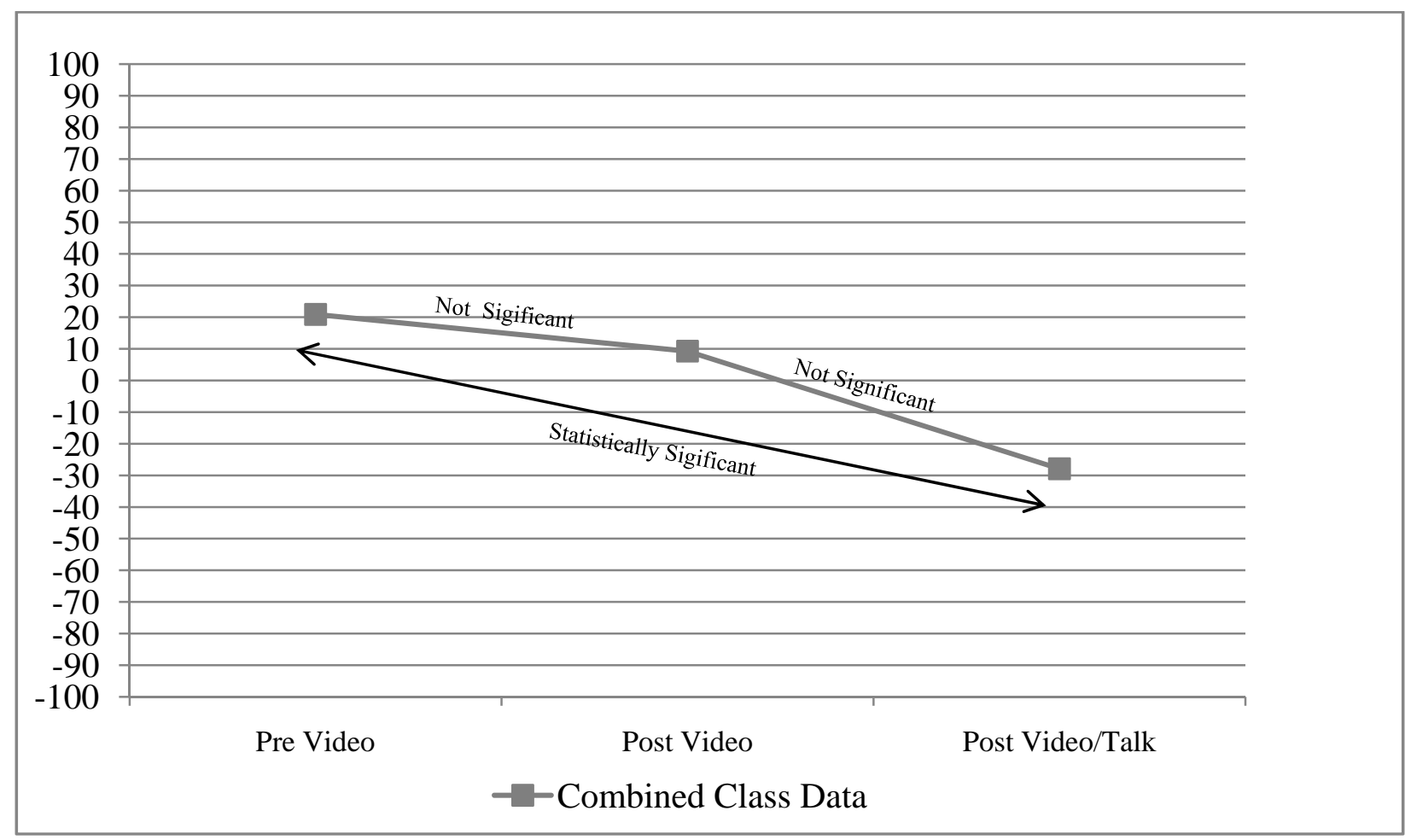

Males versus females - (c) in the beginning of this section-is divided into the same subgroups and follows the same format as (b) live versus video presentations but compares males and females overall for both treatment types.

Honors versus regular classes-(d) in the beginning of this section—-follows the same format as (c) live versus video presentations. For honors versus regular classes, individual class data were analyzed and compared for the following subgroups: pre-live versus post-live, prevideo versus post-video, post-video versus post-video + live and pre-video vs. post-video + live. Pilot study results (e) were compared to results of the current study. Only the combined classes live presentation data were analyzed within this section. All comparisons of these groups and subgroups are summarized further in Table 2. 


\section{Table 2}

Results Structure

\begin{tabular}{cc}
\hline Main Groups & Subgroups \\
\hline Pre-Treatment Comparisons & Pre-Live vs. Pre-Video \\
Honors Pre vs. Regular Pre & Males Pre vs. Females Pre \\
Combined Data (Honors and & Live vs. Video Presentations \\
Regular Classes) & Post-Video vs. Post-Video + Live \\
& Pre-Video vs. Post-Video + Live \\
Males vs. Females & Live vs. Video Presentations \\
& (Combined Classes) \\
& Post-Video vs. Post-Video + Live \\
& (Combined Classes) \\
& Pre-Video vs. Post-Video + Live \\
& (Combined Classes) \\
Honors vs. Regular Classes & Live vs. Video Presentations \\
& Post-Video vs. Post-Video + Live \\
& Pre-Video vs. Post-Video + Live \\
& Pilot Study vs. Current Study \\
& (Combined Classes) \\
\hline
\end{tabular}

In order to make the presentation of the material clearer and less mechanical to the reader, acronyms were assigned. For example, for references to questionnaire item means before any treatment, the acronym PRE will be used. Similarly, POST will be used to refer to item means after any treatment. When questionnaire item means before or after the live presentation are relevant, PRE-L and POST-L will be used, as will as PRE-V and POST-V or POST V+L following video and or follow-up video plus live treatments. Table 3 shows all acronyms and their counterparts. 
Table 3

Data Acronyms

Complete Data References

Acronyms

Questionnaire item means before live presentation

PRE-L

Questionnaire item means before video presentation

PRE-V

Questionnaire item means after live presentation

POST-L

Questionnaire item means after video presentation

POST-V

Questionnaire item means after video and shortened live presentations POST-V $+\mathrm{L}$

\section{Data Analysis}

As discussed in the methodology, all questions from the general section of the questionnaire are on a 5-point scale and all questions in the detailed section were converted to a 3-point scale as “No,” (1), “Not Sure” (2), or “Yes” (3). All means on tables and appendices were converted to a scale of -100 to 100 . -100 was deemed negative, 100 positive and 0 as neutral or undecided.

It should be noted that what is a negative or positive change depends upon the questionnaire item. For example, for the item, “People who stutter are nervous or excitable,” one would expect a positive change to go from a higher value to lower value to represent a positive change. For the item, "Stuttering is caused by genetic inheritance" one would expect a positive change to go from lower value to higher value to represent a positive, (i.e., more accurate) reflection of the state of knowledge of stuttering (Bloodstein \& Ratner, 2008). The direction of change from PRE to POST for all questionnaire items can be seen within Appendices 3-9, where ,,$+-+/-$ indicate positive, negative, or ambiguous changes. Ambiguous changes refer to certain items that are neither positive nor negative such as, "My knowledge of stuttering comes from the internet.” 
Appendix 1 shows participant’s prior experience with obesity, left handedness, stuttering, mental illness, and intelligence. Appendices 4-10 compare PRE versus POST scores for their corresponding sections. Further, a difference was calculated between PRE versus POST scores to generate an index of change. Mean scores between the two treatments were compared statistically using t-tests to evaluate the significance of any change in opinion. The Bonferroni correction was applied to probabilities for multiple t-tests to reduce the likelihood of a Type I error, i.e., rejecting the null hypothesis of no differences between the two groups when in fact the null hypothesis should not be rejected (Maxwell \& Satake, 2006). Based on a somewhat arbitrary estimate of the average of ten comparisons per cluster of items, the standard alpha level of 0.05 is divided by the number of comparisons, i.e., $0.05 / 10=0.005$, the corrected alpha level. Probabilities of $\mathrm{p}<0.05$ were indicated with *. Levels of significance were also shown for $\mathrm{p}<$ 0.005 as $* *$ and for $\mathrm{p}<0.0005$ as $* * *$.

Every section in the results presents corresponding tables that show the three largest and three smallest differences between pre versus post-means, and are sorted from greatest to least change. If there were more than three identical smallest values (usually indicating that all respondents indicated "no," which converted to -100), all smallest values were listed. Also, questionnaire items in each table are abbreviated. For example, "People who stutter are nervous or excitable" is abbreviated to "PWS: Nervous or excitable.” All abbreviations of questionnaire items can be seen in Appendix 2. Every section also has corresponding figures that show the statistically significant changes for particular sections containing at least three statistically significant changes. 


\section{Pre-Treatment}

This section shows statistically significant combined classes pre-mean data for the 45minute live presentations versus the 45-minute video presentations, males versus females, and honors versus regular classes individually. None of the comparisons between PRE-L versus PRE-V means were statistically significant, nor were comparisons between males and females, indicating that the two groups were roughly equivalent at the outset of the study.

There were, however, noticeable PRE differences between honors and regular classes. Three statistically significant differences, displayed in Figure 3, occurred between PRE means for honors and regular classes. The honors class showed more positive attitudes for items, “Cause: Learning or habits” (-11 [honors] vs. 44 [regular] and “Concern: Mom or dad” (-58 vs. 0). The honors classes seemed to generally have more positive attitudes towards stuttering before either treatment. Although remarkably, for item, “Info: From PWS” (-26 [honors] vs. 41 [regular])” the regular classes had more knowledge about stuttering from other people who stutter. Table 4 shows the three largest and smallest differences between PRE honors and regular classes. 
Figure 3. Statistically significant differences honors PRE versus regular PRE classes

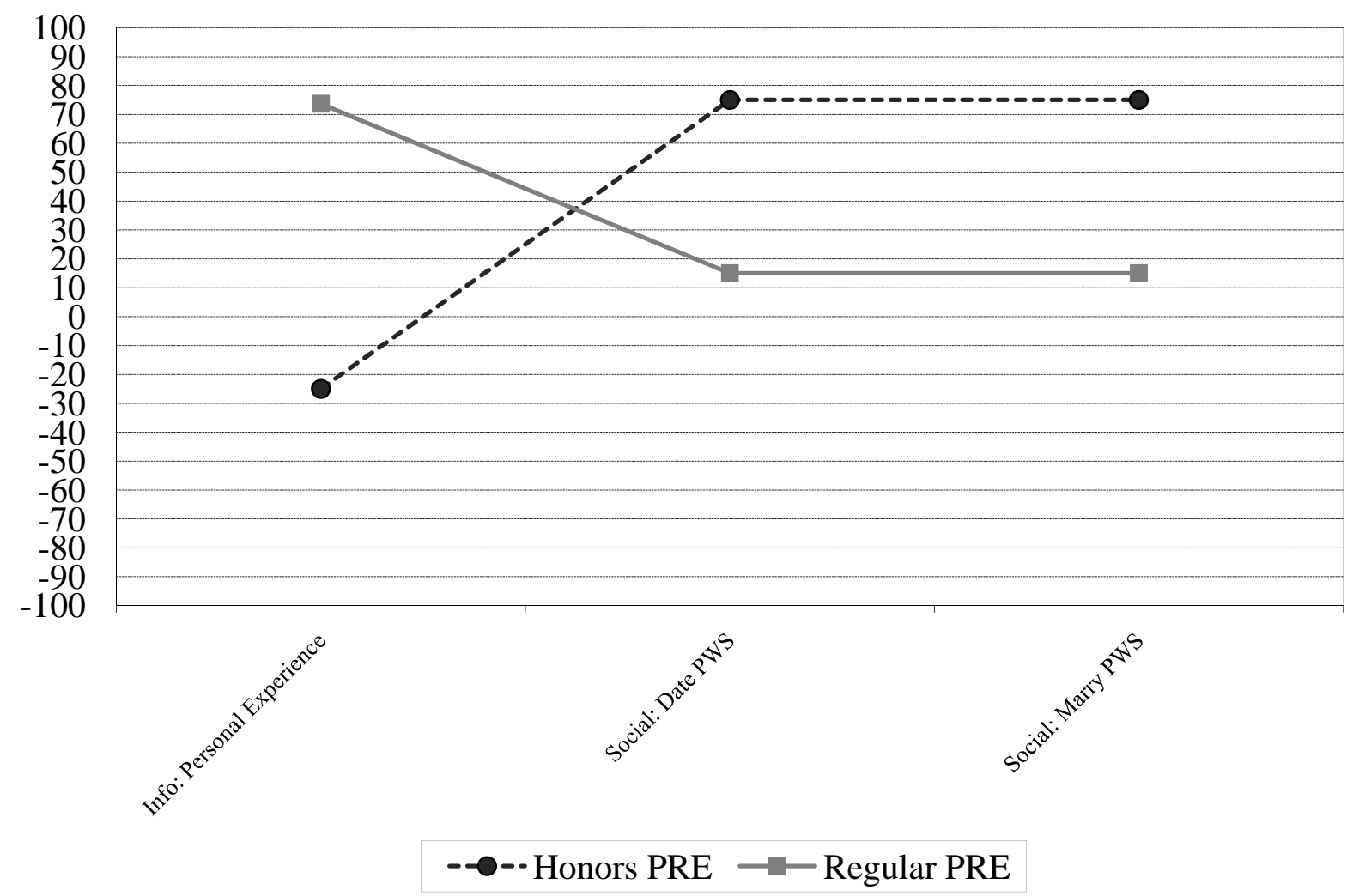

Table 4

Honors Class PRE vs. Regular Class PRE

\section{Questionnaire Items}

Largest Changes

Info: From PWS

Concern: Mom or dad

Cause: Learning or habits

Smallest Changes

Do: Ignore stuttering

Info: Magazines, newspapers, books

Want: Left handed

\section{Honors Regular \\ Pre}

$\begin{array}{lll}-26 & 41 & 67\end{array}$

$\begin{array}{lll}-58 & 0 & 58\end{array}$

$-11$

44

55

$\begin{array}{lll}92 & 91 & 1\end{array}$

$\begin{array}{lll}-34 & -34 & 0\end{array}$

$\begin{array}{lll}7 & 7 & 0\end{array}$ 


\section{Combined Data (Combined Honors and Regular Classes)}

This section shows statistically significant combined data for both pairs of classes (honors and regular) for the 45 -minute live presentation versus the 45 -minute video presentations. Fifteen POSHA-E items before versus after the live presentations and 12 before versus after video presentations were statistically significant. All statistically significant changes during the live presentations were positive, while one statistically significant change after the video presentations was negative, i.e., ratings for "I believe stuttering is caused by genetic inheritance” reduced. In addition, noticeable differences in magnitude between the live presentations and the video presentations were observed (see Appendix 4, columns 7 and 8 which lists the differences). It is clear from a comparison of the number of significant changes and the amount of change that the live presentations had a more substantial positive influence over the participants. As noted, differences between the two treatments (live and video) were observed. Figure 4 and Figure 5 show the statistically significant items and values for the live and video presentations. Table 5 shows the three largest differences, in descending order with their corresponding questions, for both live presentations on the left and for video presentations on the right followed by the three smallest differences. Table 5 concurs with the above statement that the live presentations also resulted in larger positive changes in general than the video presentations. 
Figure 4: Statistically significant changes for live presentations

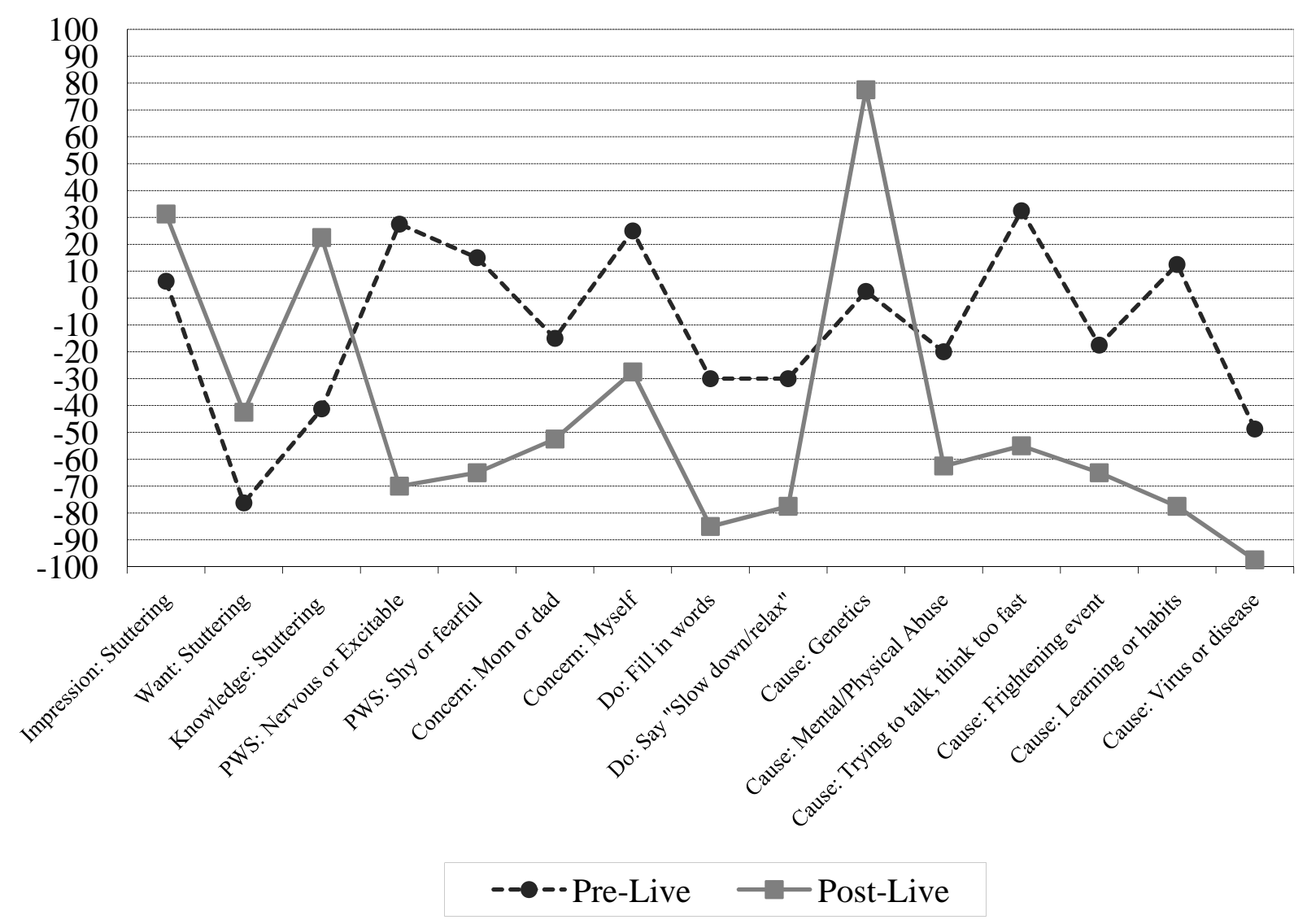


Figure 5. Statistically significant changes for video presentations

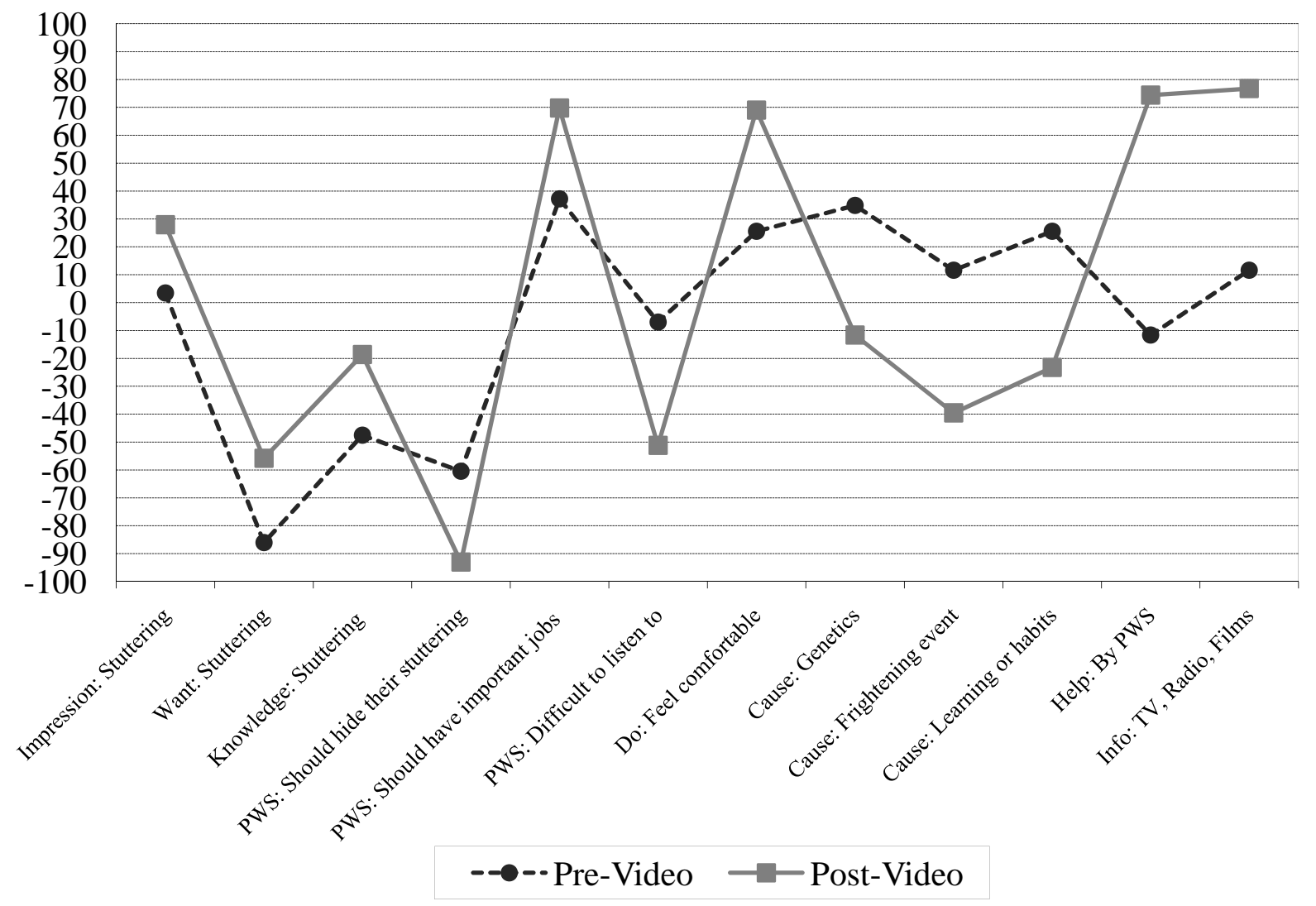


Table 5

Live vs. Video (Combined Classes): Largest and Smallest Changes.

\begin{tabular}{|c|c|c|c|c|c|c|c|}
\hline \multirow[b]{2}{*}{$\begin{array}{l}\text { Questionnaire } \\
\text { Items }\end{array}$} & \multicolumn{3}{|c|}{ Live Presentations } & \multicolumn{4}{|c|}{ Video Presentations } \\
\hline & Pre & Post & Diff & Pre & Post & Diff & $\begin{array}{l}\text { Questionnaire } \\
\text { Items: }\end{array}$ \\
\hline \multicolumn{8}{|l|}{ Largest Changes } \\
\hline $\begin{array}{l}\text { PWS: Nervous } \\
\text { or Excitable }\end{array}$ & 28 & -70 & 98 & -12 & 74 & 86 & Help: By PWS \\
\hline $\begin{array}{l}\text { Cause: Learning } \\
\text { or habits }\end{array}$ & 13 & -78 & 90 & 12 & 77 & 65 & $\begin{array}{l}\text { Info: TV, } \\
\text { Radio, Films }\end{array}$ \\
\hline $\begin{array}{l}\text { Cause: Trying } \\
\text { to talk, think too } \\
\text { fast }\end{array}$ & 33 & -55 & 88 & 12 & -40 & 51 & $\begin{array}{l}\text { Cause: } \\
\text { Frightening } \\
\text { event }\end{array}$ \\
\hline \multicolumn{8}{|l|}{ Smallest Changes } \\
\hline $\begin{array}{l}\text { Aspects: } \\
\text { Speaking ability }\end{array}$ & 70 & 70 & 0 & -99 & -97 & 2 & Want: Obese \\
\hline $\begin{array}{l}\text { Do: Feel } \\
\text { comfortable }\end{array}$ & 63 & 63 & 0 & 100 & 100 & 0 & $\begin{array}{l}\text { PWS: Can } \\
\text { make friends }\end{array}$ \\
\hline $\begin{array}{l}\text { Cause: Ghosts, } \\
\text { demons, spirits }\end{array}$ & -100 & -100 & 0 & -91 & -91 & 0 & $\begin{array}{l}\text { Cause: Ghosts, } \\
\text { demons, spirits }\end{array}$ \\
\hline
\end{tabular}

Video Presentations vs. Video + Live (Combined Classes)

Next, for the video treatment, comparisons were made between respondents’ second and third POSHA-Es completed after the video and then after the follow-up shortened live presentations. Figures 6 and 7 shows the combined data for both the honors and the regular classes for the POST-V versus POST-V+L and the PRE-V versus the POST-V+L. Five statistically significant changes, all positive, were observed for the POST-V versus POST-V+L. Statistically significant changes for the PRE-V versus POST-V+L occurred for 17 items, and all were positive. Generally, the shortened live presentation further positively impacted attitudes and reversed significant negative changes from PRE versus POST-V presentation. For example, in the item, “I believe stuttering is caused by genetic inheritance,” mean ratings reduced from 35 
(pre-video) to -12 (post-video), but then extended to 74 (post shortened live presentation). This can be seen in the beginning of the results section in Figure 1 .

Figure 6. Combined classes statistically significant changes post-video vs. post-video + live

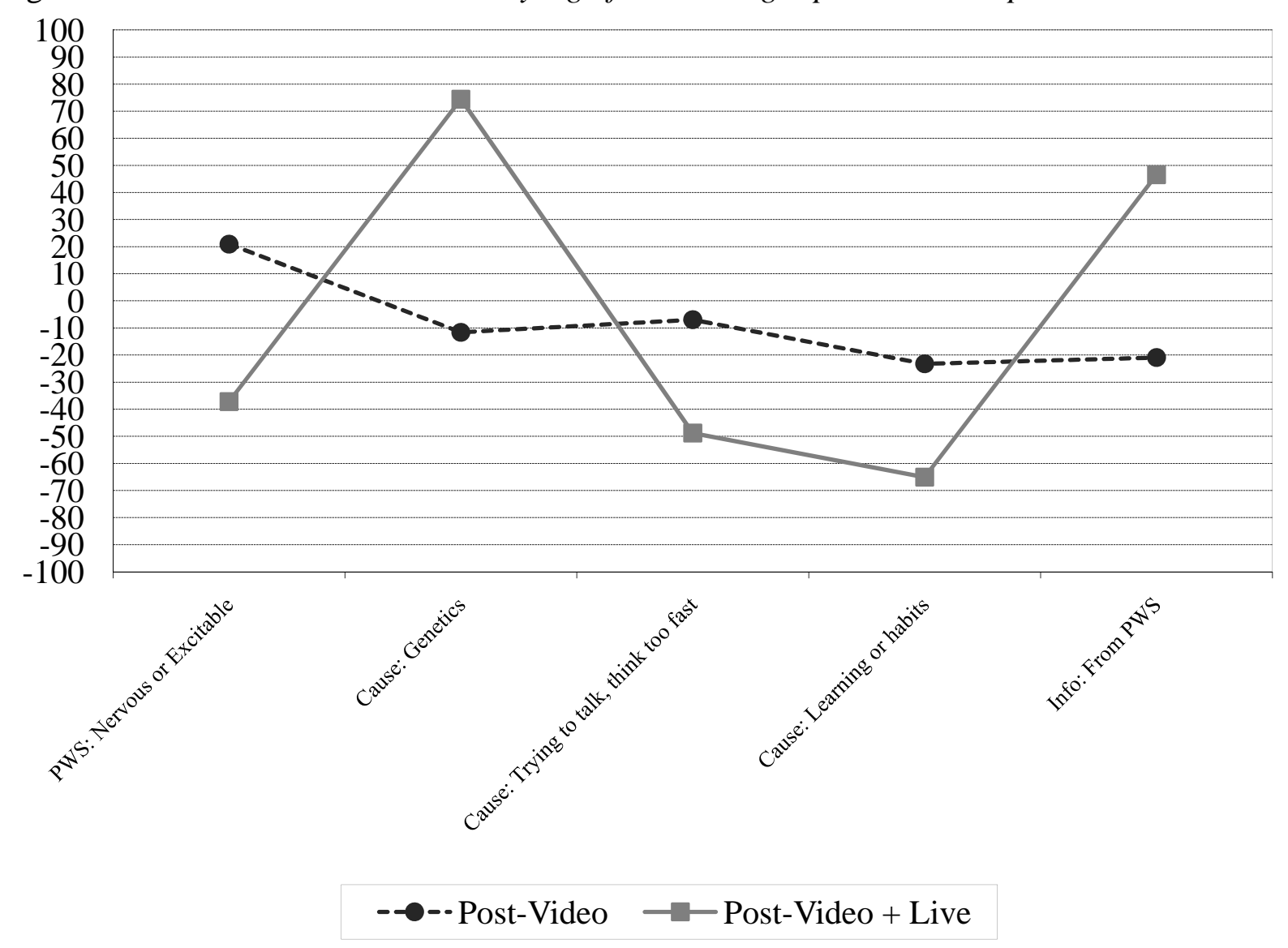


Figure 7. Combined classes statistically significant changes pre-video vs. post-video + live

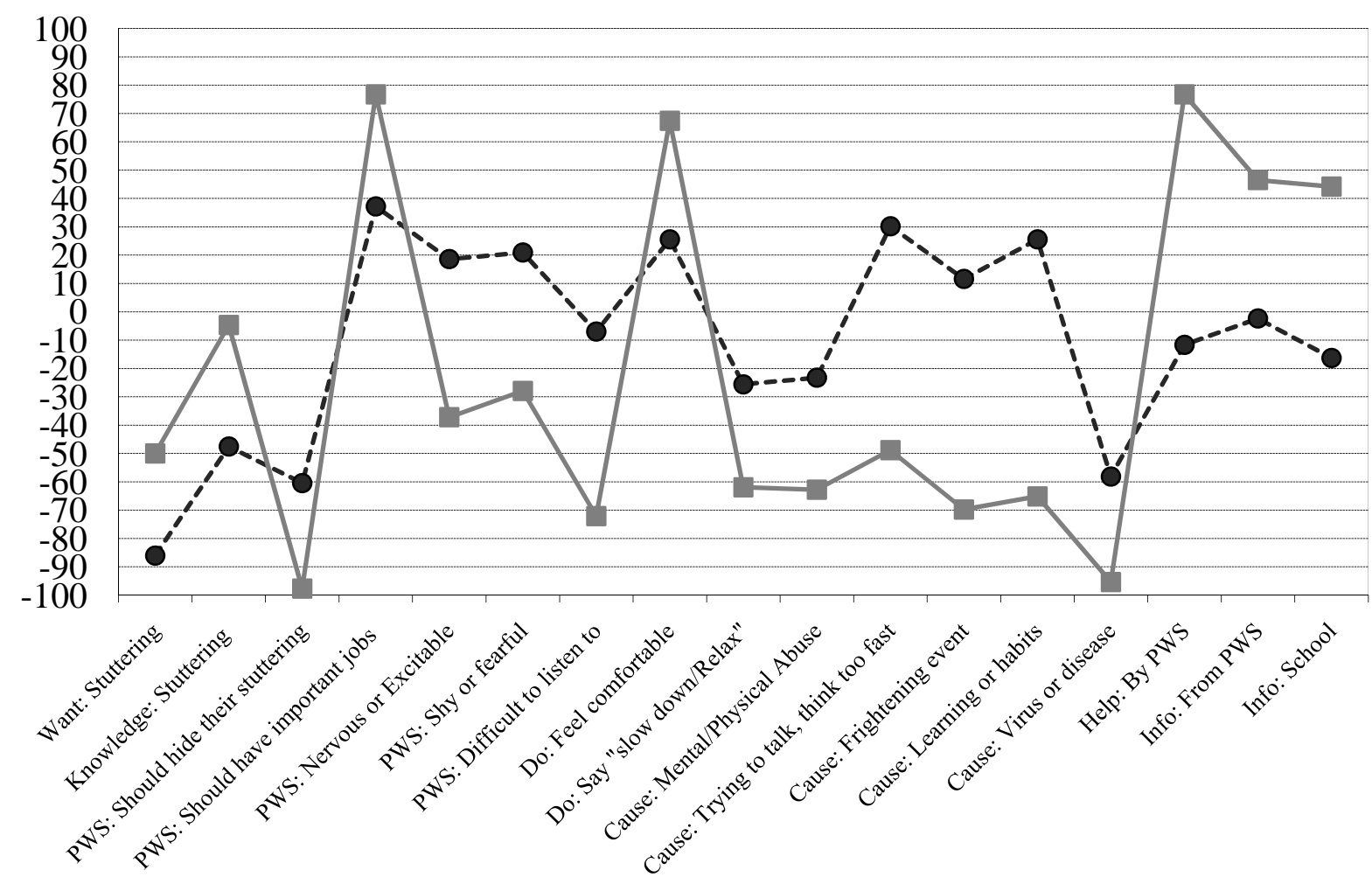

- ๑--Pre-Video $\rightarrow$ - Post-Video + Live

Table 6 shows the three largest differences along with the smallest differences for POST$\mathrm{V}$ versus POST-V + $\mathrm{L}$ and PRE-V versus POST-V + L. POST-V versus POST-V+L had more items with identical smallest differences (8), whereas the PRE-V versus POST-V+L had 4 items with identical smallest differences, i.e., no change from PRE to POST. In interpreting individual items, it should be noted that items varied relative to the degree of which mean scores could change. A "ceiling effect" or "floor effect" can affect mean difference scores if the basal level has already reached—or nearly reached—the highest or lowest possible value. For example, while "Social: Talk to PWS” and "Social: Friends with PWS" show zero difference for POST-V 
versus POST-V+L, these two questionnaire items could not increase positively since, at the PRE-

V state, the maximum mean scores were 100 .

Table 6

Post-Video vs. Post-Video + Live, Pre-Video vs. Post-Video + Live (Combined Classes):

Largest and Smallest Changes

\begin{tabular}{|c|c|c|c|c|c|c|c|}
\hline \multirow[b]{2}{*}{$\begin{array}{l}\text { Questionnaire } \\
\text { Items: }\end{array}$} & \multicolumn{3}{|c|}{ Post-Video vs. Post-V+L } & \multicolumn{4}{|c|}{ Pre-Video vs. Post-V + L } \\
\hline & $\begin{array}{l}\text { Post } \\
\text { Vid }\end{array}$ & $\begin{array}{l}\text { Post } \\
\text { V+L }\end{array}$ & Diff & Pre & $\begin{array}{l}\text { Post } \\
\text { V+L }\end{array}$ & Diff & $\begin{array}{l}\text { Questionnaire } \\
\text { Items: }\end{array}$ \\
\hline Largest Changes & & & & & & & Largest Changes \\
\hline Cause: Genetics & -12 & 74 & 86 & 26 & -65 & 91 & $\begin{array}{l}\text { Cause: Learning } \\
\text { or habits }\end{array}$ \\
\hline Info: From PWS & -21 & 47 & 67 & -12 & 77 & 88 & Help: By PWS \\
\hline $\begin{array}{l}\text { PWS: Nervous or } \\
\text { Excitable }\end{array}$ & 21 & -37 & 58 & 12 & -70 & 81 & $\begin{array}{l}\text { Cause: } \\
\text { Frightening event }\end{array}$ \\
\hline Smallest Changes & & & & & & & Smallest Changes \\
\hline $\begin{array}{l}\text { Want: Mentally } \\
\text { ill }\end{array}$ & -84 & -84 & 0 & -- & -- & -- & -- \\
\hline $\begin{array}{l}\text { Knowledge: } \\
\text { Intelligent }\end{array}$ & 30 & 30 & 0 & -- & -- & -- & -- \\
\hline $\begin{array}{l}\text { PWS: Can make } \\
\text { friends }\end{array}$ & 100 & 100 & 0 & -- & -- & -- & -- \\
\hline Concern: Doctor & -67 & -67 & 0 & -- & -- & -- & -- \\
\hline $\begin{array}{l}\text { Concern: Brother } \\
\text { or sister }\end{array}$ & -63 & -63 & 0 & 72 & 72 & 0 & $\begin{array}{l}\text { Aspects: Learn } \\
\text { new things }\end{array}$ \\
\hline Help: By SLP & 93 & 93 & 0 & 7 & 7 & 0 & $\begin{array}{l}\text { Want: Left } \\
\text { handed }\end{array}$ \\
\hline $\begin{array}{l}\text { Social: Talk to } \\
\text { PWS }\end{array}$ & 100 & 100 & 0 & 100 & 100 & 0 & $\begin{array}{l}\text { PWS: Can make } \\
\text { friends }\end{array}$ \\
\hline $\begin{array}{l}\text { Social: Friends } \\
\text { with PWS }\end{array}$ & 100 & 100 & 0 & 98 & 98 & 0 & $\begin{array}{l}\text { PWS: Lead } \\
\text { normal lives }\end{array}$ \\
\hline
\end{tabular}

-- refers to an uneven number of identical smallest differences between POST-V vs. POST-V+L and PRE-V vs. POST-V+L 


\section{Males Versus Females \\ Live Presentations}

There were a total of 15 statistically significant changes (four for males and 11 for females) for the live presentations, all of which were positive. While the live presentation affected both males and females positively, female’s attitude changes were greater. Both the number of statistically significant changes (four statistically significant changes for males versus 11 for females) and the amount of change favored females. Figure 8 and 9 show the statistically significant changes for both male and females during the 45-minute live presentation.

Figure 8. Males statistically significant changes live presentations

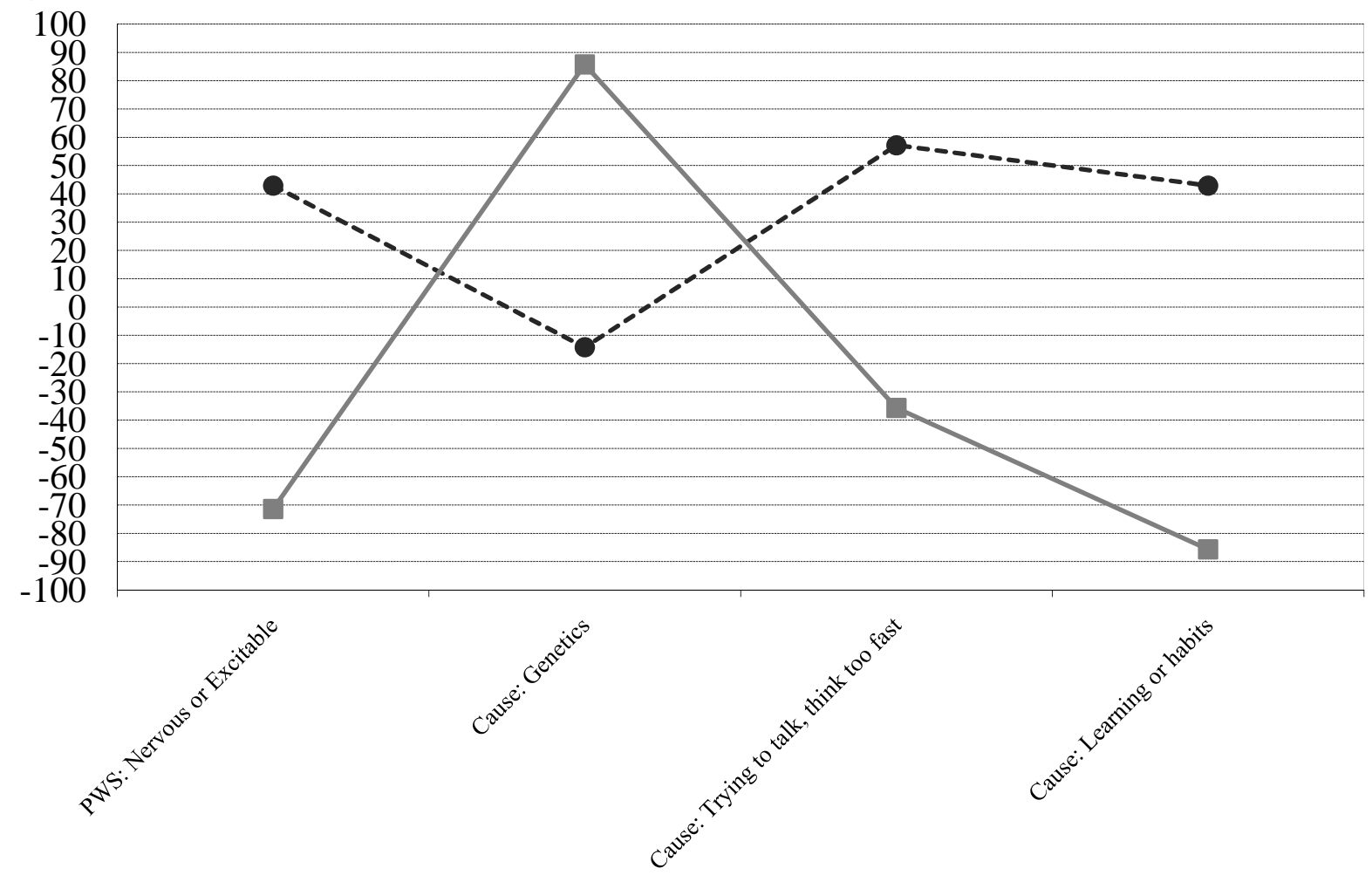

$$
\text { -๑--Pre-Live } \longrightarrow \text { Post-Live }
$$


Figure 9. Female statistically significant changes live presentations

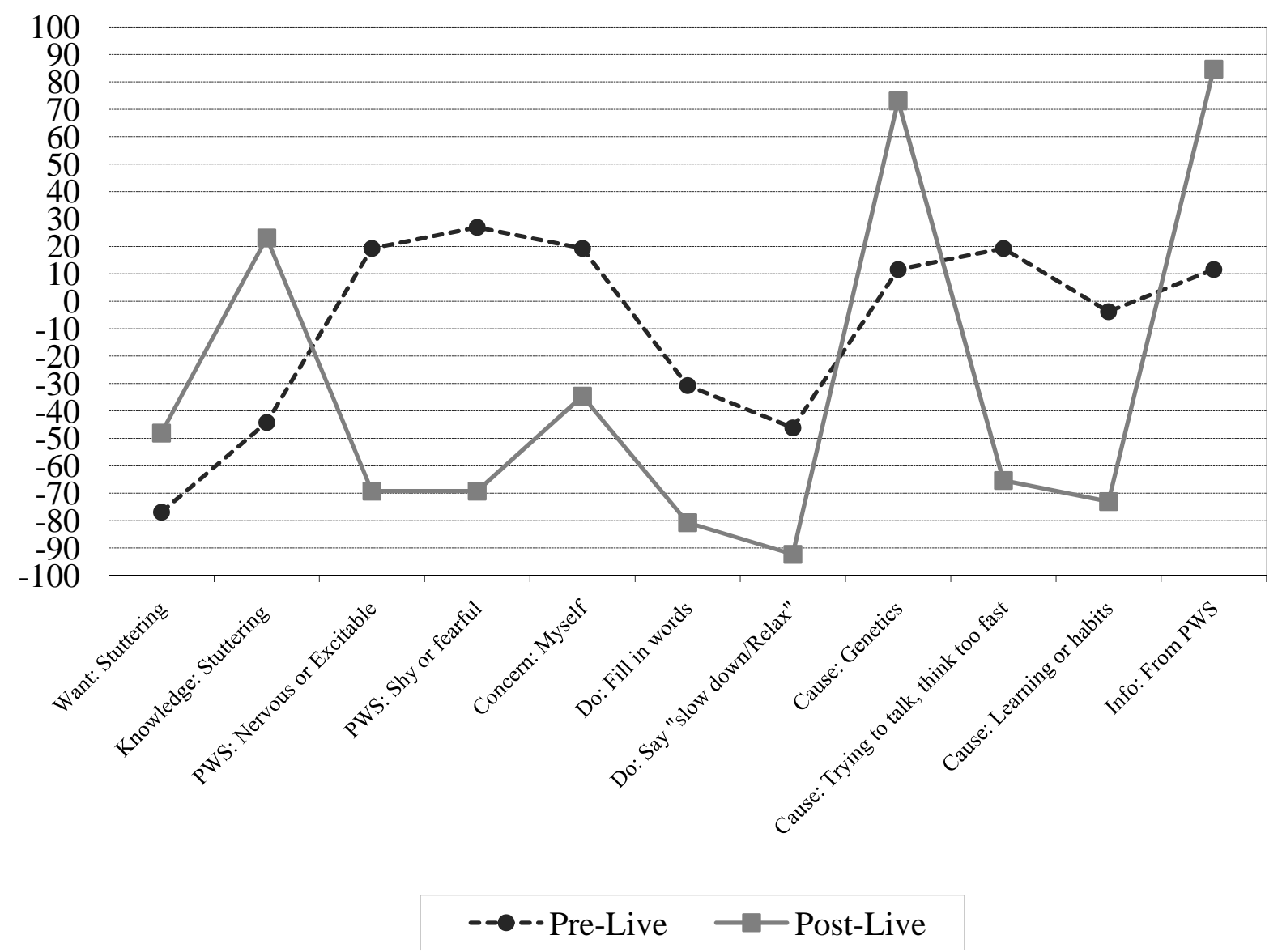

Table 7 shows the three largest and smallest PRE versus POST differences between males versus females before and after the 45-minute live presentation (combined classes). Males showed a larger difference from PRE to POST in part because of a stronger negative attitude. The questionnaire item, "PWS: Nervous or Excitable,” (43 vs. -71 [males], 19 vs. -69 [females]) reflected a stronger PRE score for males, although after the live presentation, male and females POST scores were almost exactly the same. Males showed a more negative PRE score than females for the questionnaire item, “Cause: Learning or habits” (43 vs. -86 [male], -4 vs. -73 [female]) while POST scores were comparable to one another. 
Table 7

Male vs. Females Live Presentations (Combined Classes)

\begin{tabular}{|c|c|c|c|c|c|c|c|}
\hline \multirow[b]{2}{*}{$\begin{array}{l}\text { Questionnaire } \\
\text { Items: }\end{array}$} & \multicolumn{2}{|c|}{ Male } & \multirow[b]{2}{*}{ Diff } & \multicolumn{2}{|c|}{ Female } & \multirow[b]{2}{*}{ Diff } & \multirow[b]{2}{*}{$\begin{array}{l}\text { Questionnaire } \\
\text { Items: }\end{array}$} \\
\hline & $\begin{array}{l}\text { Pre } \\
\text { Live }\end{array}$ & $\begin{array}{l}\text { Post } \\
\text { Live }\end{array}$ & & $\begin{array}{l}\text { Pre } \\
\text { Live }\end{array}$ & $\begin{array}{l}\text { Post } \\
\text { Live }\end{array}$ & & \\
\hline Largest Changes & & & & & & & Largest Changes \\
\hline $\begin{array}{l}\text { Cause: Learning or } \\
\text { habits }\end{array}$ & 43 & -86 & 129 & 27 & -69 & 96 & $\begin{array}{l}\text { PWS: Shy or } \\
\text { fearful }\end{array}$ \\
\hline $\begin{array}{l}\text { PWS: Nervous or } \\
\text { Excitable }\end{array}$ & 43 & -71 & 114 & 19 & -69 & 88 & $\begin{array}{l}\text { PWS: Nervous or } \\
\text { Excitable }\end{array}$ \\
\hline Cause: Genetics & -14 & 86 & 100 & 19 & -65 & 85 & $\begin{array}{l}\text { Cause: Trying to } \\
\text { talk, think too fast }\end{array}$ \\
\hline Smallest Changes & & & & & & & Smallest Changes \\
\hline-- & -- & -- & -- & 100 & 100 & 0 & $\begin{array}{l}\text { PWS: Lead normal } \\
\text { lives }\end{array}$ \\
\hline $\begin{array}{l}\text { Aspects: Learn } \\
\text { new things }\end{array}$ & 82 & 82 & 0 & 62 & 62 & 0 & $\begin{array}{l}\text { Do: Feel } \\
\text { comfortable }\end{array}$ \\
\hline Want : Intelligent & 61 & 61 & 0 & -100 & -100 & 0 & $\begin{array}{l}\text { Cause: Ghosts, } \\
\text { demons, spirits }\end{array}$ \\
\hline $\begin{array}{l}\text { PWS: Can make } \\
\text { friends }\end{array}$ & 100 & 100 & 0 & 4 & 4 & 0 & Help: By PWS \\
\hline $\begin{array}{l}\text { Do: Feel } \\
\text { comfortable }\end{array}$ & 64 & 64 & 0 & -12 & -12 & 0 & Help: By doctor \\
\hline $\begin{array}{l}\text { Cause: Ghosts, } \\
\text { demons, spirits }\end{array}$ & -100 & -100 & 0 & 65 & 65 & 0 & Social: Marry PWS \\
\hline
\end{tabular}

-- refers to an uneven number of identical smallest differences between males and females for PRE-L vs. POST-L (Combined Classes)

\section{Video Presentation}

As in the live presentations, there were significant differences between males and females for the combined video presentations (honors class and regular class). For males, only one statistically significant change occurred for the item, "Info: TV, radio, films” (-11 vs. 68). It was therefore not graphed. By contrast, female ratings for seven items changed by statistically significant amounts after watching the True Life: I Stutter video. Figure 10 shows the statistically 
significant changes for females before and after the video presentations. Overall the attitudes of males after the video presentations generally changed in the same direction as those for females, but the magnitude of females' attitude changes were greater. The item, "PWS: Difficult to listen to” clearly illustrates this point, i.e., -16 vs. -21 for males and 0 vs. -75 for females. Both male and female PRE scores were comparable while POST scores were considerably different. There were also nonsignificant examples where male and female PRE versus POST results changed in different directions as in the item, "PWS: Are nervous or excitable" (32 vs. 53 [males], 8 vs. -4 [females]) and “Do: Fill in words” (-63 vs. -37 [males], -50 vs. -63 [females]). While the video did not significantly alter attitudes for either sex in this particular item, males generally regarded PWS as more nervous after viewing the video, while females generally thought PWS were slightly less nervous after viewing the video. Females appeared to be more receptive to the video presentation than males. 
Figure 10. Females statistically significant changes video presentations

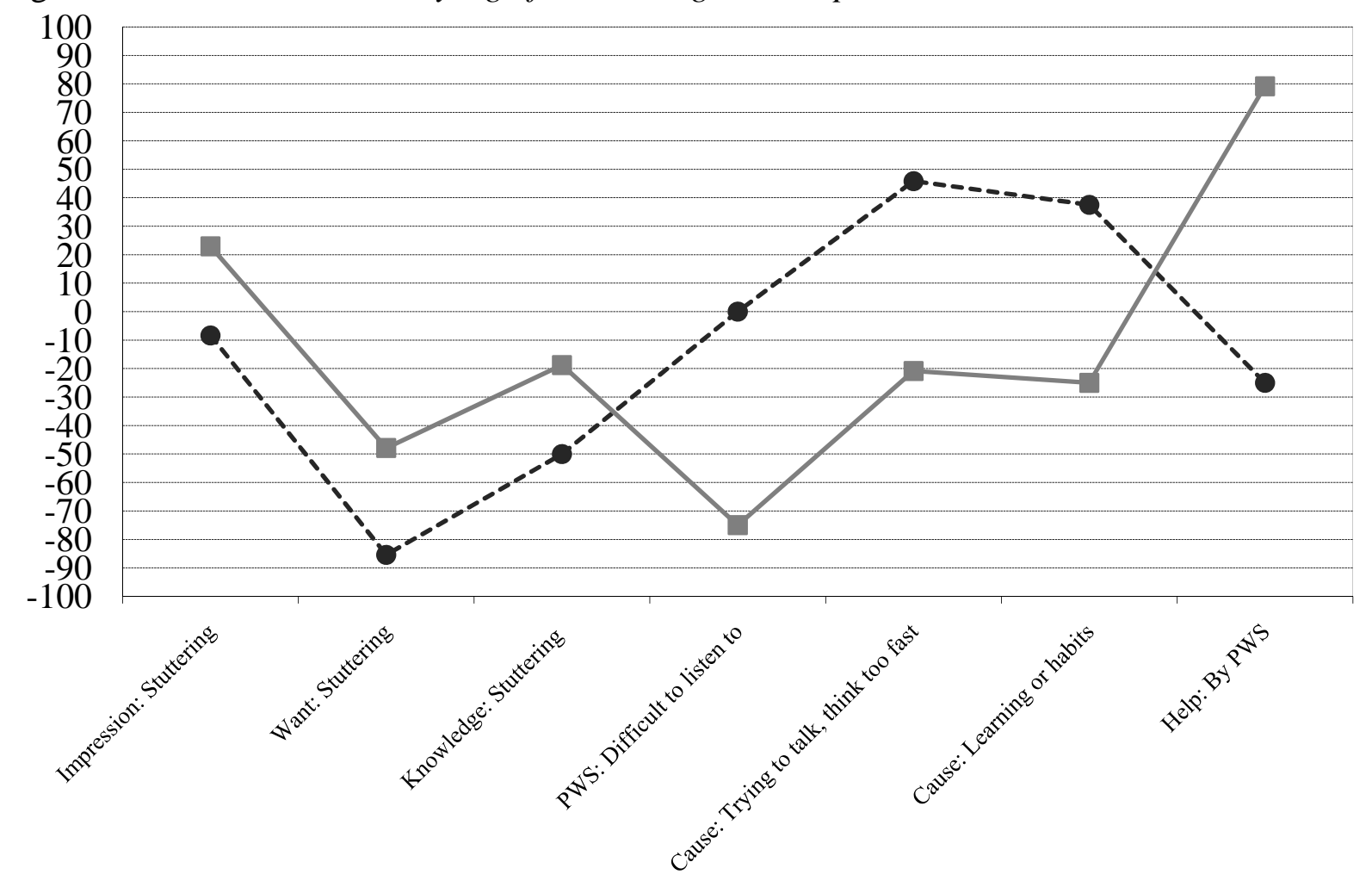

Table 8 shows the largest and smallest differences for PRE versus POST for both males and females before and after the video presentations. The video presentations had a different effect than that of the live presentations. For the live presentations, male negative attitudes were stronger, and therefore led to a greater difference between PRE and POST scores. For the video presentations, male attitudes showed stronger negative attitudes, but the video did little to alter these attitudes, resulting in smaller differences from PRE to POST scores. 
Table 8

Males vs. Females 45-Minute Video Presentation (Combined Classes)

\begin{tabular}{|c|c|c|c|c|c|c|c|}
\hline \multirow[b]{2}{*}{$\begin{array}{l}\text { Questionnaire } \\
\text { Items: }\end{array}$} & \multicolumn{2}{|c|}{ Male } & \multirow[b]{2}{*}{ Diff } & \multicolumn{2}{|c|}{ Female } & \multirow[b]{2}{*}{ Diff } & \multirow[b]{2}{*}{$\begin{array}{l}\text { Questionnaire } \\
\text { Items: }\end{array}$} \\
\hline & $\begin{array}{l}\text { Pre } \\
\text { Vid } \\
\end{array}$ & $\begin{array}{c}\text { Post } \\
\text { Vid } \\
\end{array}$ & & $\begin{array}{l}\text { Pre } \\
\text { Vid }\end{array}$ & $\begin{array}{l}\text { Post } \\
\text { Vid } \\
\end{array}$ & & \\
\hline Largest Changes & & & & & & & Largest Changes \\
\hline $\begin{array}{l}\text { Info: TV, Radio, } \\
\text { Films }\end{array}$ & -11 & 68 & 79 & -25 & 79 & 104 & Help: By PWS \\
\hline Info: School & -16 & 47 & 63 & 0 & -75 & 75 & $\begin{array}{l}\text { PWS: Difficult to } \\
\text { listen to }\end{array}$ \\
\hline Help: By PWS & 5 & 68 & 63 & 46 & -21 & 67 & $\begin{array}{l}\text { Cause: Trying to } \\
\text { talk, think too fast }\end{array}$ \\
\hline Smallest Changes & & & & & & & Smallest Changes \\
\hline Want: Mentally ill & -92 & -92 & 0 & -- & -- & -- & -- \\
\hline $\begin{array}{l}\text { PWS: Can make } \\
\text { friends }\end{array}$ & 100 & 100 & 0 & -- & -- & -- & -- \\
\hline Concern: Doctor & -63 & -63 & 0 & 69 & 69 & 0 & $\begin{array}{l}\text { Aspects: Learn } \\
\text { new things }\end{array}$ \\
\hline $\begin{array}{l}\text { Do: Ignore } \\
\text { stuttering }\end{array}$ & 84 & 84 & 0 & 100 & 100 & 0 & $\begin{array}{l}\text { PWS: Can make } \\
\text { friends }\end{array}$ \\
\hline $\begin{array}{l}\text { Do: Joke about } \\
\text { stuttering }\end{array}$ & -100 & -100 & 0 & 71 & 71 & 0 & $\begin{array}{l}\text { PWS: Can do any } \\
\text { job they want }\end{array}$ \\
\hline $\begin{array}{l}\text { Do: Say "slow } \\
\text { down/Relax" }\end{array}$ & -32 & -32 & 0 & -92 & -92 & 0 & $\begin{array}{l}\text { Concern: } \\
\text { Neighbor }\end{array}$ \\
\hline $\begin{array}{l}\text { Cause: Trying to } \\
\text { talk, think too fast }\end{array}$ & 11 & 11 & 0 & 92 & 92 & 0 & Help: By SLP \\
\hline
\end{tabular}

-- refers to an uneven number of identical smallest differences between males and females for PRE-V vs. POST-V (Combined Classes)

\section{Post-Video Versus Post-Video + Live}

There were similarities in the number of statistically significant changes for males and females for POST-V versus POST-V+L, i.e., three versus two. The items, "PWS: Nervous or Excitable” (53 vs. -26 [males], -4 vs. -46 [females]) and “Cause: Genetic Inheritance” (-21 vs. 84 [males], -4 vs. 67 [females]) were significant for both males and females. The shortened live 
presentation (20 minutes) further positively influenced males for the items, "Cause: Learning or habits” (11 [pre vid] vs. -21 [post vid] vs. -74 [post vid + live]) making it statistically significant from POST-V versus POST-V+L. Figure 18 and 19 show the statistically significant changes for males and females for POST-V versus POST- $\mathrm{V}+\mathrm{L}$. As explained in the introduction, these further changes in attitudes after the post-video live presentation attest to the importance of carrying out the POST-V versus POST-V+L comparisons.

Figure 11. Males statistically significant changes post-video versus post-video + live

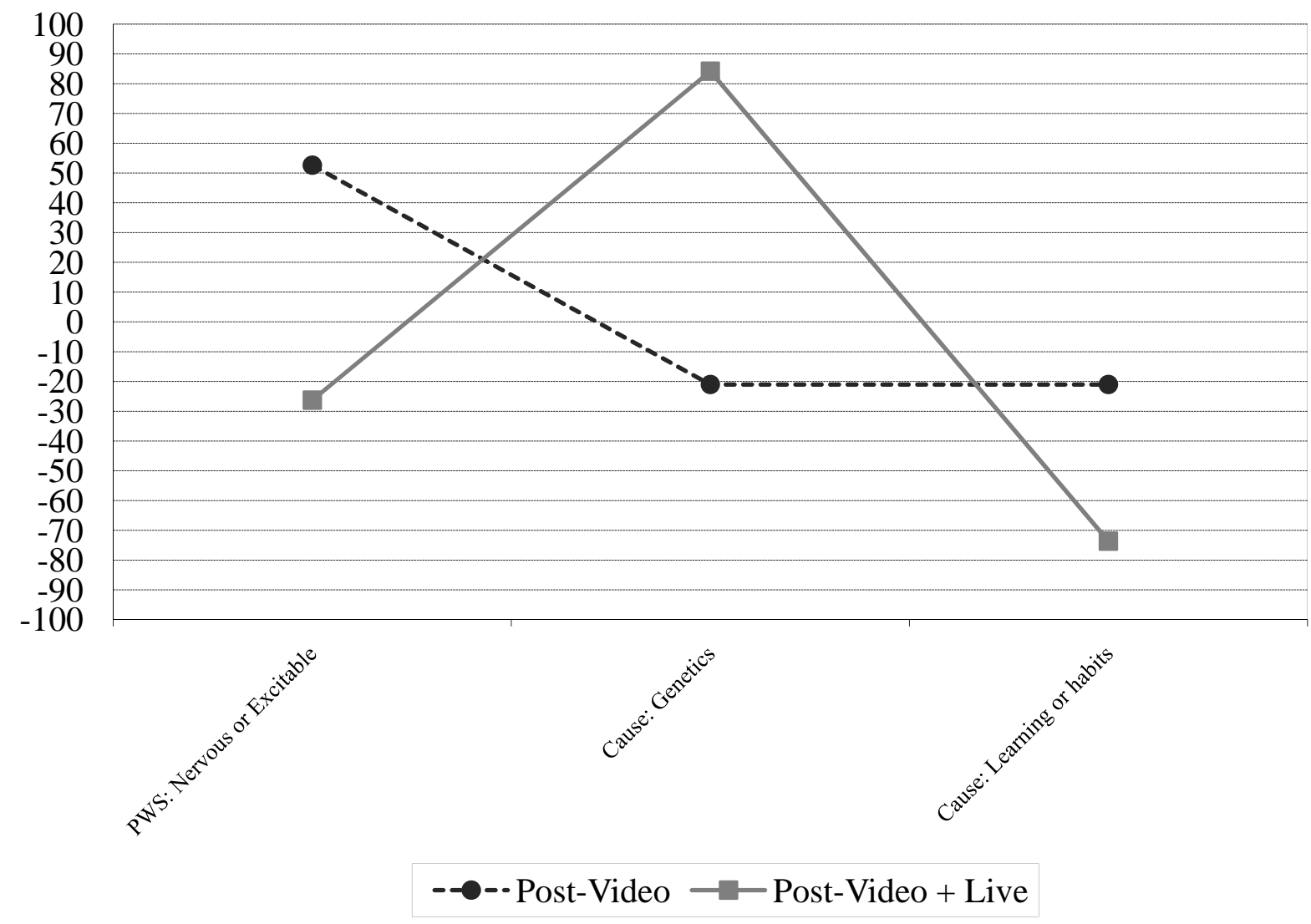


Figure 12. Females statistically significant changes post-video versus post-video + live
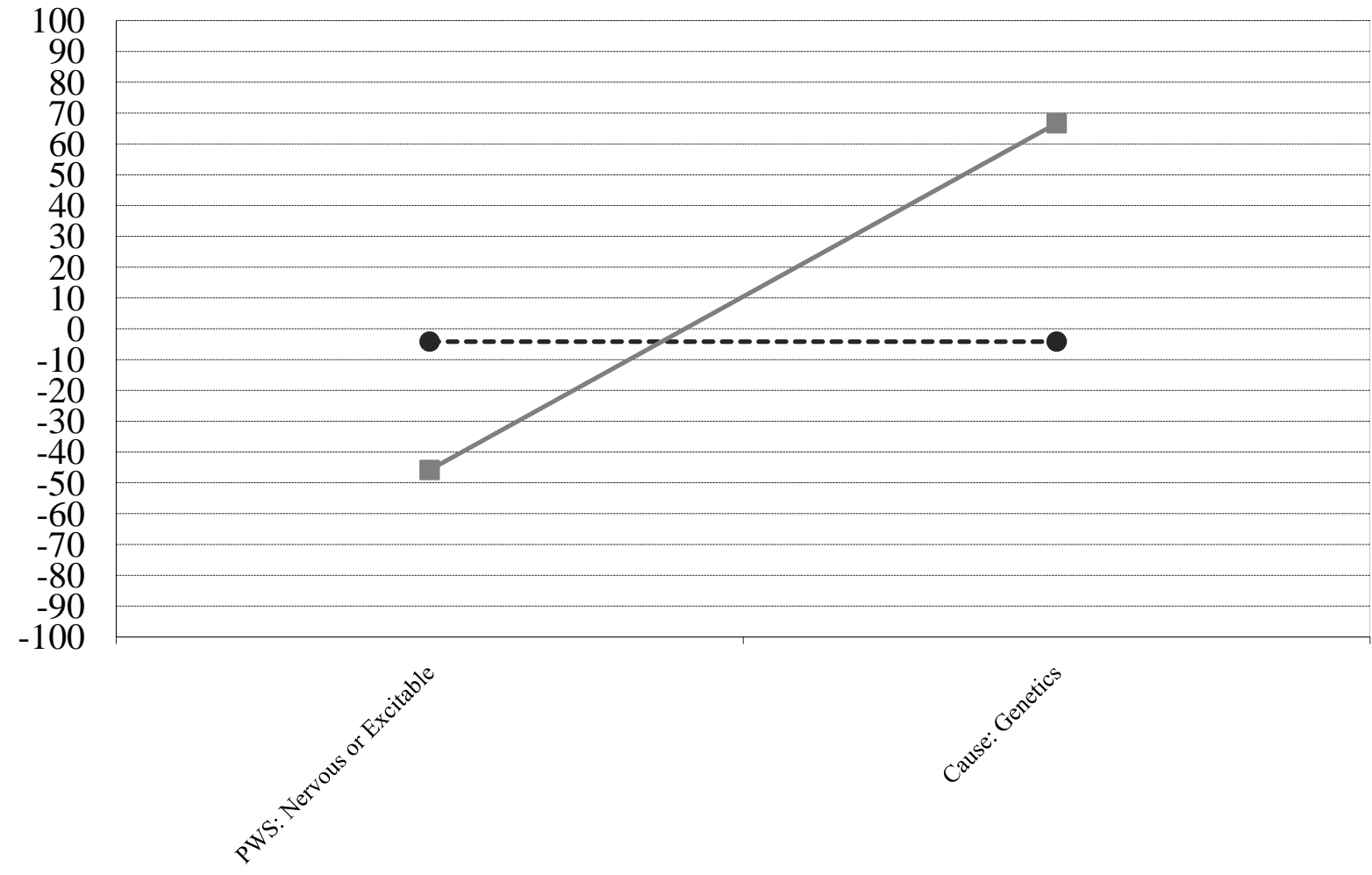

--๑--Post-Video $\longrightarrow$ - Post-Video + Live

Table 9 shows the largest and smallest differences for POST-V versus POST-V+L for both males and females. Males and females' top three largest differences were for the same questionnaire items: “Cause: Genetics,” "PWS: Nervous or excitable,” and “Info: From PWS.” Males once again made larger changes than females for changes after the shortened live presentation, most likely due to stronger negative attitudes after viewing the video. After viewing the video, males rated PWS as more nervous, while females rated them as less nervous. Both males and females responded positively after the shortened live presentation for "PWS: Nervous or excitable", reversing the effects of the video for males, and further positively influencing females. 
Table 9

Males vs. Females Post-Video vs. Post-Video + Live (Combined Classes)

\begin{tabular}{|c|c|c|c|c|c|c|c|}
\hline $\begin{array}{l}\text { Questionnaire } \\
\text { Items: }\end{array}$ & $\begin{array}{l}\text { Post- } \\
\text { video }\end{array}$ & $\begin{array}{c}\text { Male } \\
\text { Post } \\
\text { V+L } \\
\end{array}$ & Diff & $\begin{array}{l}\text { Post- } \\
\text { video }\end{array}$ & $\begin{array}{l}\text { emale } \\
\text { Post } \\
\text { V+L }\end{array}$ & Diff & $\begin{array}{l}\text { Questionnaire } \\
\text { Items: }\end{array}$ \\
\hline Largest Changes & & & & & & & Largest Changes \\
\hline Cause: Genetics & -21 & 84 & 105 & -4 & 67 & 71 & Cause: Genetics \\
\hline $\begin{array}{l}\text { PWS: Nervous or } \\
\text { Excitable }\end{array}$ & 53 & -26 & 79 & -29 & 38 & 67 & Info: From PWS \\
\hline Info: From PWS & -11 & 58 & 69 & -4 & -46 & 42 & $\begin{array}{l}\text { PWS: Nervous or } \\
\text { Excitable }\end{array}$ \\
\hline Smallest Changes & & & & & & & Smallest Changes \\
\hline $\begin{array}{l}\text { PWS: Should } \\
\text { have important } \\
\text { jobs }\end{array}$ & 68 & 68 & 0 & -- & -- & -- & -- \\
\hline $\begin{array}{l}\text { PWS: Can make } \\
\text { friends }\end{array}$ & 100 & 100 & 0 & -- & -- & -- & -- \\
\hline $\begin{array}{l}\text { PWS: Can do any } \\
\text { job they want }\end{array}$ & 100 & 100 & 0 & 69 & 69 & 0 & $\begin{array}{l}\text { Aspects: Learn } \\
\text { new things }\end{array}$ \\
\hline $\begin{array}{l}\text { Concern: } \\
\text { Neighbor }\end{array}$ & -95 & -95 & 0 & -98 & -98 & 0 & Want: Obese \\
\hline $\begin{array}{l}\text { Do: Joke about } \\
\text { stuttering }\end{array}$ & -100 & -100 & 0 & -96 & -96 & 0 & $\begin{array}{l}\text { PWS: Should } \\
\text { hide their } \\
\text { stuttering }\end{array}$ \\
\hline $\begin{array}{l}\text { Do: Feel } \\
\text { impatient }\end{array}$ & -84 & -84 & 0 & 100 & 100 & 0 & $\begin{array}{l}\text { PWS: Can make } \\
\text { friends }\end{array}$ \\
\hline Help: By PWS & 68 & 68 & 0 & -75 & -75 & 0 & $\begin{array}{l}\text { PWS: Difficult to } \\
\text { listen to }\end{array}$ \\
\hline Help: By SLP & 95 & 95 & 0 & -100 & -100 & 0 & $\begin{array}{l}\text { Cause: Ghosts, } \\
\text { demons, spirits }\end{array}$ \\
\hline $\begin{array}{l}\text { Social: Talk to } \\
\text { PWS }\end{array}$ & 100 & 100 & 0 & 92 & 92 & 0 & Help: By SLP \\
\hline $\begin{array}{l}\text { Social: Friends } \\
\text { with PWS }\end{array}$ & 100 & 100 & 0 & 100 & 100 & 0 & $\begin{array}{l}\text { Social: Talk to } \\
\text { PWS }\end{array}$ \\
\hline
\end{tabular}

-- refers to an uneven number of identical smallest differences between males and females for POST-V vs. POST-V+L (Combined Classes) 


\section{Pre-Video Versus Post-Video + Live}

There were eight statistically significant changes for males and eleven statistically significant changes for females before the video presentation versus after the video and shortened live presentation. Receiving both the video presentation and the shortened live presentation influenced both males and females in a positive manner. The shortened live presentation reversed most of the negative changes from the video presentation for both sexes and had a further positive influence. Males especially showed a large difference between PRE-V versus POST-V (one significant change) and PRE-V versus POST-V+L (eight significant changes). The shortened live presentation also had a greater positive impact on males and females than the video. The shortened live presentation had a greater impact on males than females as well. Figure 13 and 14 show the statistically significant changes for males and females for PRE-V versus POST-V+L. 
Figure 13. Males statistically significant changes pre-video versus post-video + live

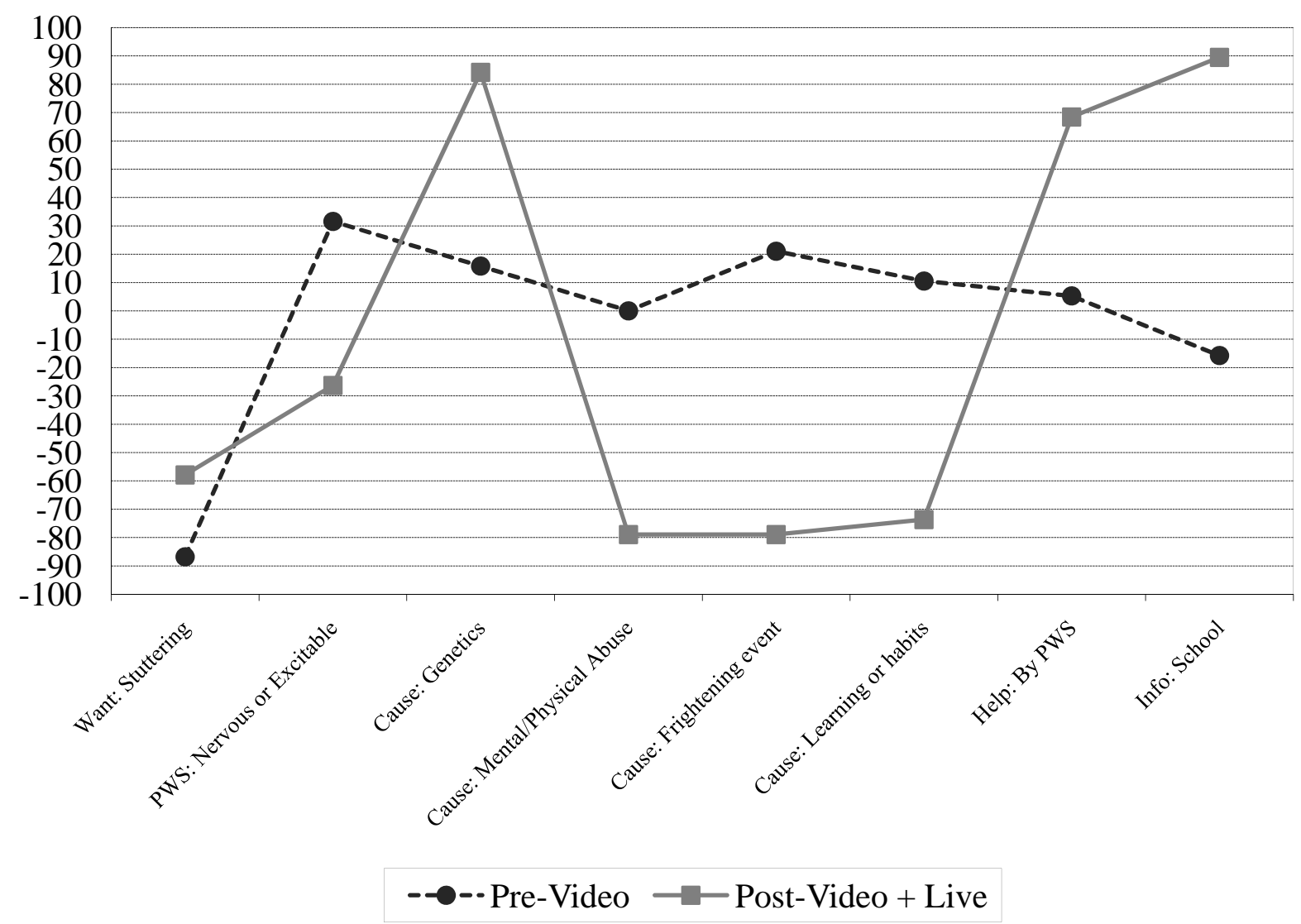


Figure 14. Females statistically significant changes pre-video versus post-video + live

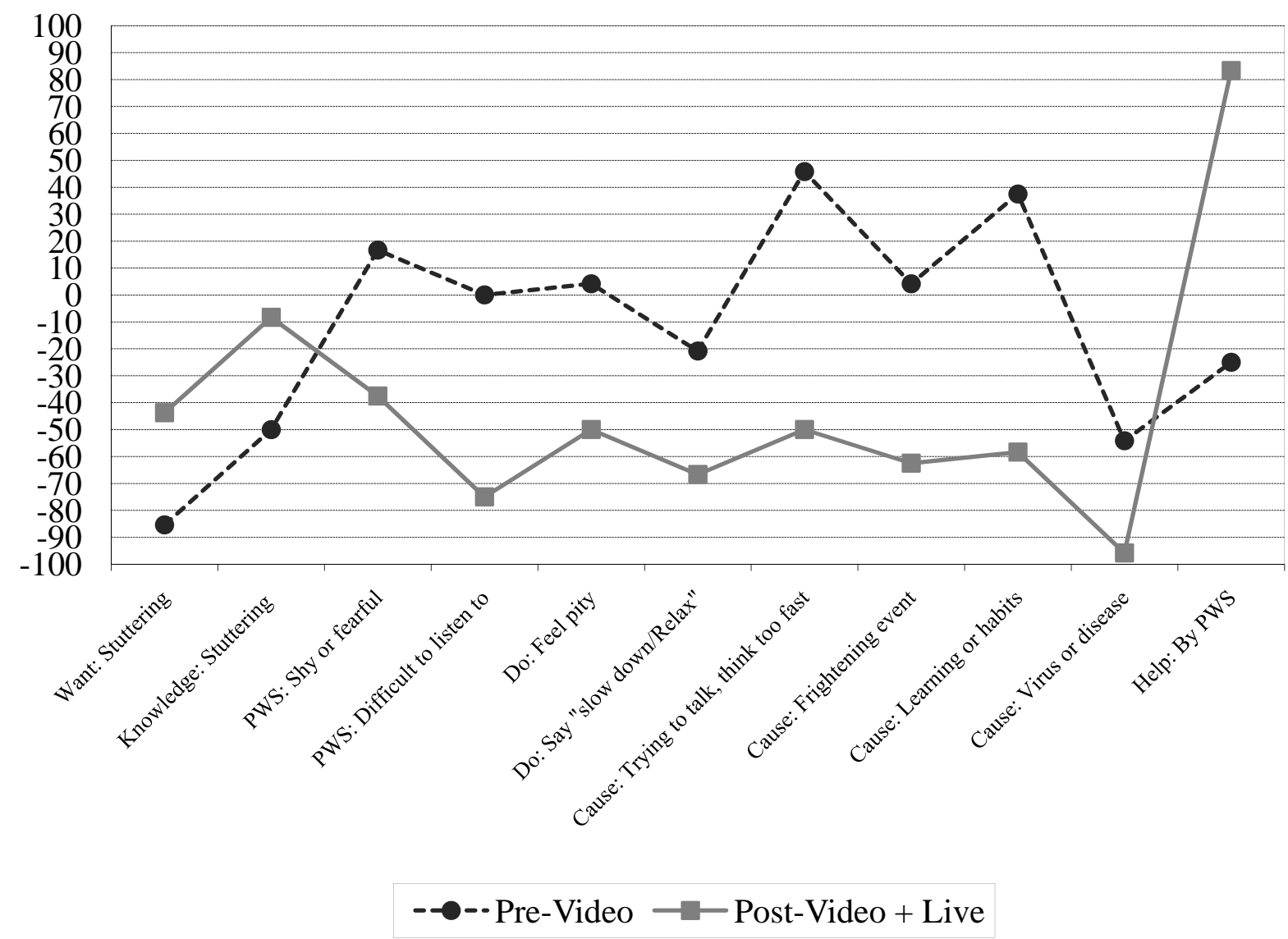

Table 10 shows the three largest differences and smallest differences for PRE- versus POST-V+L for males and females. While males generally had stronger negative attitudes beforehand (PRE), their POST scores were more positive than females, e.g., in: "Cause: Mental/Physical Abuse” (0 vs. -79 [males], -42 vs. -50 [females]), “Cause: Frightening event” (21 vs. -79 [males], 4 vs. -63 [females]), “Cause: Genetics” (16 vs. 84 [males], 50 vs. 67 [females]), and “Do: Feel impatient” (-68 vs. -84 [males], -50 vs. -79 [females]) (Refer to Appendix 6). 
Table 10

Males vs. Females Pre-Video vs. Post-Video + Live (Combined Classes)

\begin{tabular}{|c|c|c|c|c|c|c|c|}
\hline \multirow[b]{2}{*}{$\begin{array}{l}\text { Questionnaire } \\
\text { Items: }\end{array}$} & \multicolumn{3}{|c|}{ Male } & \multicolumn{4}{|c|}{ Female } \\
\hline & $\begin{array}{l}\text { Pre } \\
\text { Vid }\end{array}$ & $\begin{array}{l}\text { Post } \\
\text { V+L }\end{array}$ & Diff & $\begin{array}{l}\text { Pre } \\
\text { Vid }\end{array}$ & $\begin{array}{l}\text { Post } \\
\text { V+L }\end{array}$ & Diff & $\begin{array}{l}\text { Questionnaire } \\
\text { Items: }\end{array}$ \\
\hline Largest Changes & & & & & & & Largest Changes \\
\hline Info: School & -16 & 89 & 105 & -25 & 83 & 108 & Help: By PWS \\
\hline $\begin{array}{l}\text { Cause: } \\
\text { Frightening event }\end{array}$ & 21 & -79 & 100 & 46 & -50 & 96 & $\begin{array}{l}\text { Cause: Trying to } \\
\text { talk, think too } \\
\text { fast }\end{array}$ \\
\hline $\begin{array}{l}\text { Cause: Learning } \\
\text { or habits }\end{array}$ & 11 & -74 & 85 & 38 & -58 & 96 & $\begin{array}{l}\text { Cause: Learning } \\
\text { or habits }\end{array}$ \\
\hline Smallest Changes & & & & & & & Smallest Changes \\
\hline-- & -- & -- & -- & 69 & 69 & 0 & $\begin{array}{l}\text { Aspects: Learn } \\
\text { new things }\end{array}$ \\
\hline -- & -- & -- & -- & 100 & 100 & 0 & $\begin{array}{l}\text { PWS: Can make } \\
\text { friends }\end{array}$ \\
\hline $\begin{array}{l}\text { Aspects: Learn } \\
\text { new things }\end{array}$ & 76 & 76 & 0 & 96 & 96 & 0 & $\begin{array}{l}\text { PWS: Lead } \\
\text { normal lives }\end{array}$ \\
\hline $\begin{array}{l}\text { Impression: } \\
\text { Obese }\end{array}$ & -24 & -24 & 0 & -50 & -50 & 0 & $\begin{array}{l}\text { Concern: Brother } \\
\text { or sister }\end{array}$ \\
\hline $\begin{array}{l}\text { PWS: Can make } \\
\text { friends }\end{array}$ & 100 & 100 & 0 & 96 & 96 & 0 & $\begin{array}{l}\text { Do: Ignore } \\
\text { stuttering }\end{array}$ \\
\hline $\begin{array}{l}\text { PWS: Lead } \\
\text { normal lives }\end{array}$ & 100 & 100 & 0 & 92 & 92 & 0 & Help: By SLP \\
\hline $\begin{array}{l}\text { Do: Joke about } \\
\text { stuttering }\end{array}$ & -100 & -100 & 0 & -38 & -38 & 0 & $\begin{array}{l}\text { Info: Magazines, } \\
\text { newspapers, } \\
\text { books }\end{array}$ \\
\hline
\end{tabular}

-- refers to an uneven number of identical smallest differences between males and females for PRE-V vs. POST-V+L (Combined Classes) 


\section{Honors vs. Regular Classes}

\section{Live Presentations}

Nine statistically significant positive changes were observed for the honors class and seven for the regular class. While some of these significant changes were shared between both classes, others were not. Ratings for the item, "PWS: Shy or fearful” (0 vs. -90 [honors], 30 vs. -40 [regular]) in the honors class PRE versus POST are significant while the regular classes are not. The regular class weakened a stronger stereotype to a moderate degree while the honors class altered a weaker stereotype to an almost non-existent one. For the item, "Do: Fill in words" (-50 vs. -95 [honors], -10 vs.-75 [regular]) the regular class once again has a less desirable PRE score but a statistically significant POST score. This is in part due to the regular class having a less desirable PRE score. Appendix 7 shows the regular class's stronger negative attitudes or less desirable reactions to stuttering for nearly all questionnaire items in both PRE and POST scores when compared to the honors class. This can be seen in several items: "PWS: Should hide their stuttering” (-80 vs. -95 [honors], -55 vs. -75 [regular]), “PWS: Nervous or excitable” (20 vs. -85 [honors], 35 vs. -55 [regular]), and “Do: Feel pity” (-5 vs. -45 [honors], 30 vs. -5[regular]). One of the few exceptions was for the item, "PWS: Should have important jobs” (47 vs. 50 [honors], 25 vs. 55 [regular]). In this item, the regular class once again had a less desirable PRE score but a more desirable POST score after hearing the live presentation, but only by a small amount. Figure 15 and 16 show the statistically significant changes for regular and honors classes for the live presentation. 
Figure 15. Honors class pre-live versus post-live presentation

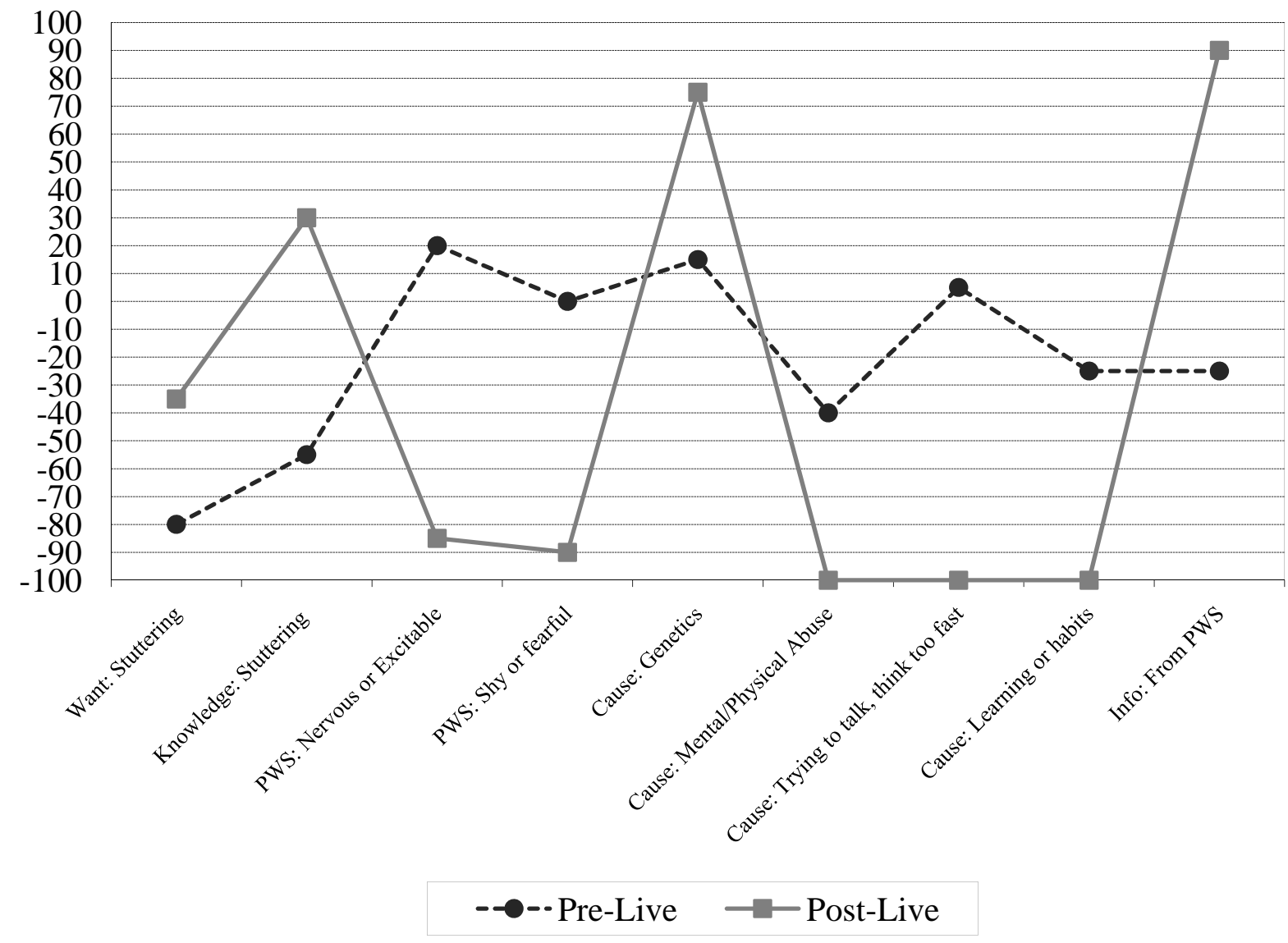


Figure 16. Regular class pre-live versus post-live presentation

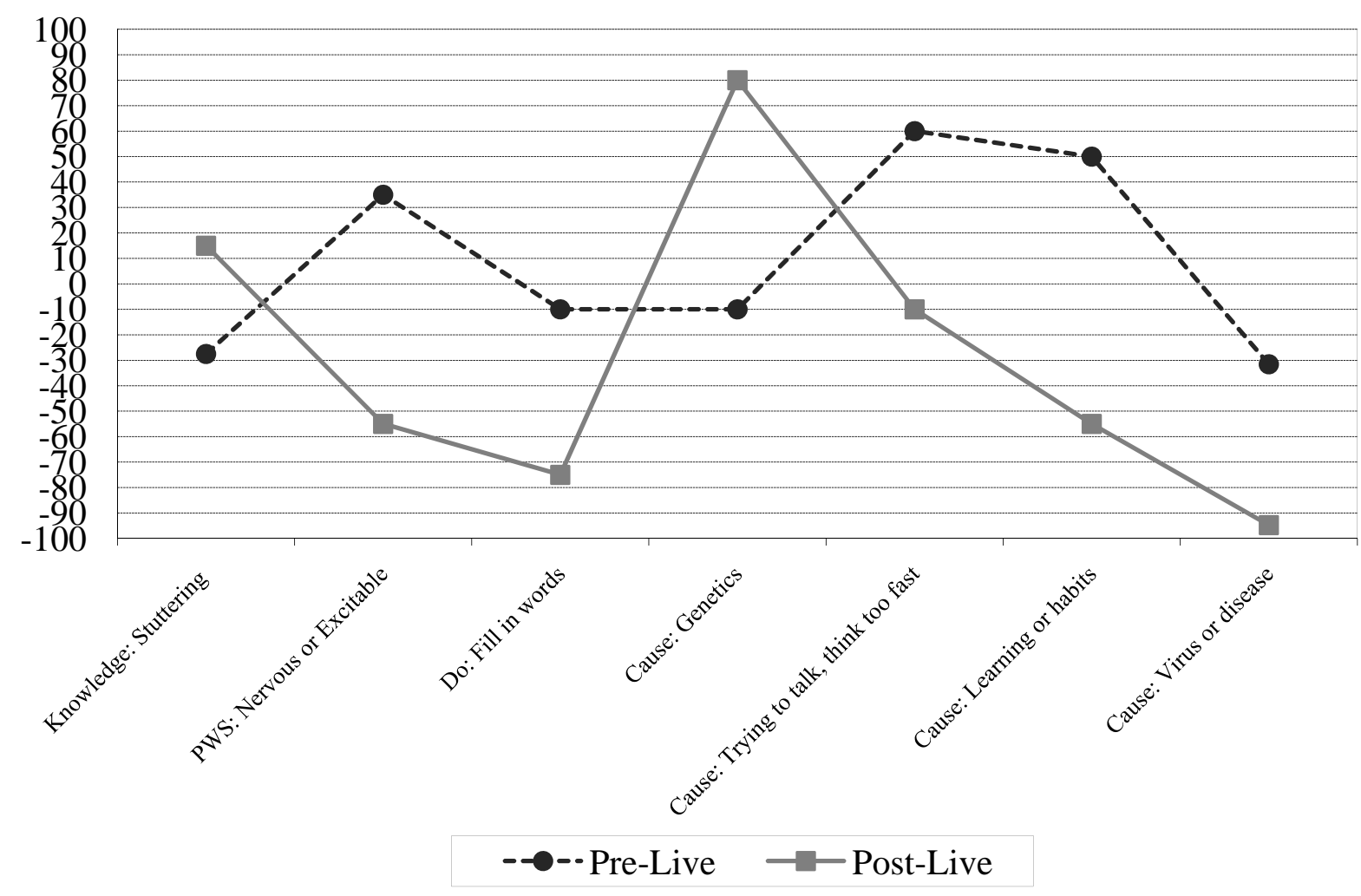

Table 11 shows the three largest differences for honors versus regular classes for the live presentation along with the smallest differences. The regular class generally had more negative attitudes prior to the live presentation than the honors class, and were not impacted as much by the presentations. 
Table 11

Honors and Regular Class Live Presentation

\begin{tabular}{|c|c|c|c|c|c|c|c|}
\hline \multirow[b]{2}{*}{$\begin{array}{l}\text { Questionnaire } \\
\text { Items: }\end{array}$} & \multicolumn{3}{|c|}{ Honors Class } & \multicolumn{4}{|c|}{ Regular Class } \\
\hline & Pre & Post & Diff & Pre & Post & Diff & $\begin{array}{l}\text { Questionnaire } \\
\text { Items: }\end{array}$ \\
\hline Largest Changes & & & & & & & Largest Changes \\
\hline Info: From PWS & -25 & 90 & 115 & 50 & -55 & 105 & $\begin{array}{l}\text { Cause: Learning } \\
\text { or habits }\end{array}$ \\
\hline $\begin{array}{l}\text { PWS: Nervous or } \\
\text { Excitable }\end{array}$ & 20 & -85 & 105 & 35 & -55 & 90 & $\begin{array}{l}\text { PWS: Nervous or } \\
\text { Excitable }\end{array}$ \\
\hline $\begin{array}{l}\text { Cause: Trying to } \\
\text { talk, think too fast }\end{array}$ & 5 & -100 & 105 & -10 & 80 & 90 & Cause: Genetics \\
\hline Smallest Changes & & & & & & & Smallest Changes \\
\hline-- & -- & -- & -- & 80 & 80 & 0 & $\begin{array}{l}\text { Aspects: Mental } \\
\text { health }\end{array}$ \\
\hline $\begin{array}{l}\text { Knowledge: } \\
\text { Obese }\end{array}$ & 8 & 8 & 0 & 100 & 100 & 0 & $\begin{array}{l}\text { PWS: Can make } \\
\text { friends }\end{array}$ \\
\hline $\begin{array}{l}\text { PWS: Lead } \\
\text { normal lives }\end{array}$ & 100 & 100 & 0 & -85 & -85 & 0 & $\begin{array}{l}\text { Concern: } \\
\text { Neighbor }\end{array}$ \\
\hline $\begin{array}{l}\text { Cause: Ghosts, } \\
\text { demons, spirits }\end{array}$ & -100 & -100 & 0 & -100 & -100 & 0 & $\begin{array}{l}\text { Cause: Ghosts, } \\
\text { demons, spirits }\end{array}$ \\
\hline
\end{tabular}

-- refers to an uneven number of identical smallest differences between honors class and regular class

\section{Video Presentations}

A total of 13 statistically significant changes took place between the honors and regular classes after the video presentations, seven and six respectively. All of the statistically significant changes were positive except in the honors class for the questionnaire item, "Cause: Genetics" (33 vs. -44). As observed in previous sections, the video presentation reduced what participants in both honors and regular classes believed the cause of stuttering to be. All items in the stuttering etiology section of the questionnaire were either reduced from PRE to POST or remained the same. As with the live presentations, the honors class again generally showed 
stronger positive attitudes after the video presentations than those of the regular class, but neither class reacted to the video significantly more than the other. For the item, "PWS: Can do any job they want to do" (83 vs. 78 [honors], 68 vs. 88 [regular]), the honors class had a more positive PRE score, but the regular class had a more positive POST score. For the item, "PWS: Are difficult to listen to" (11 vs. -67 [honors], -20 vs. -40 [regular], the regular class had a more positive PRE score, but the honors class has a more positive POST score. Figure 17 and 18 show the statistically significant changes for the PRE-V versus POST-V presentation for the honors and regular classes.

Figure 17. Honors class pre-video versus post-video presentation

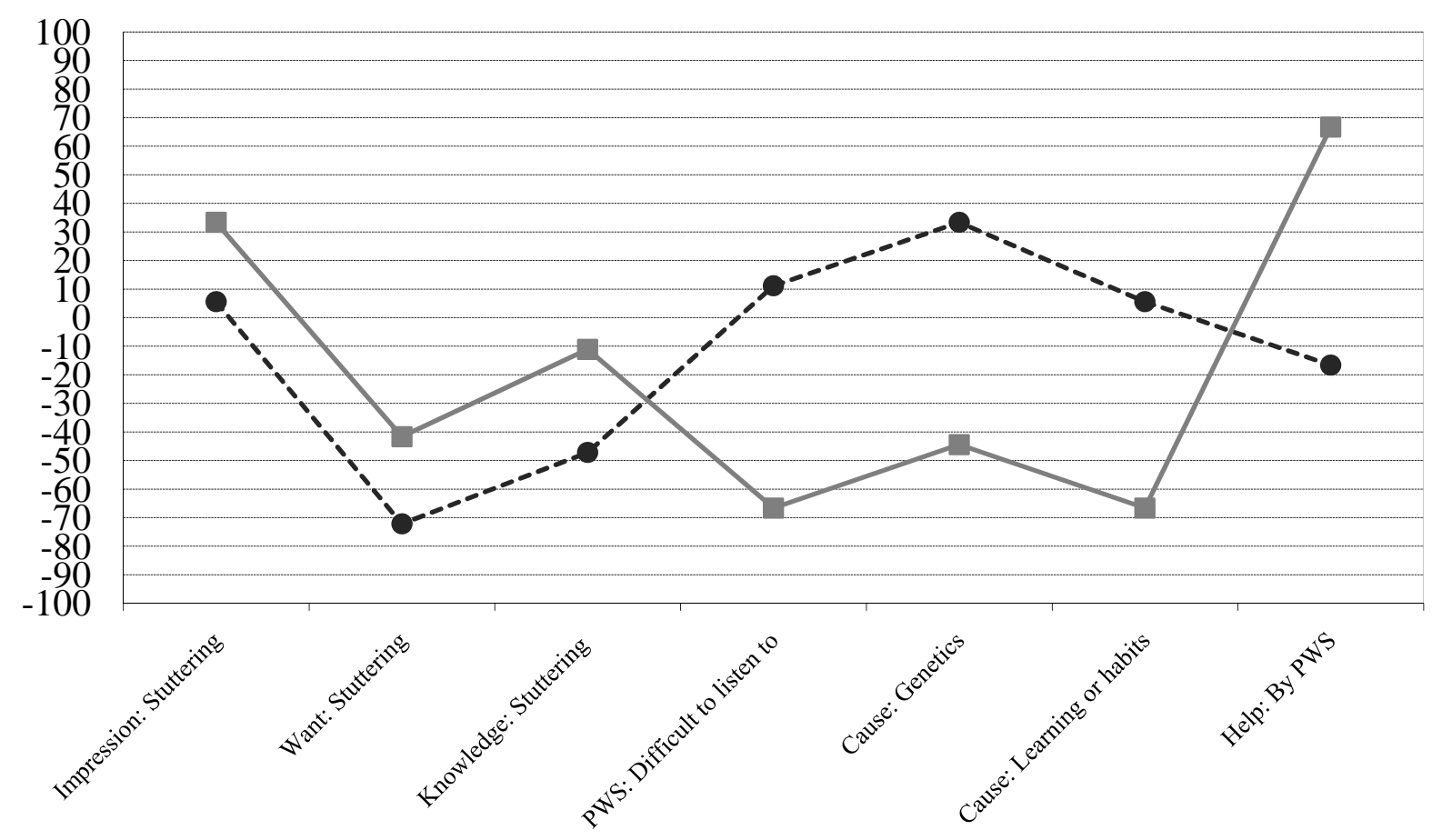


Figure 18. Regular class pre-video versus post-video presentation

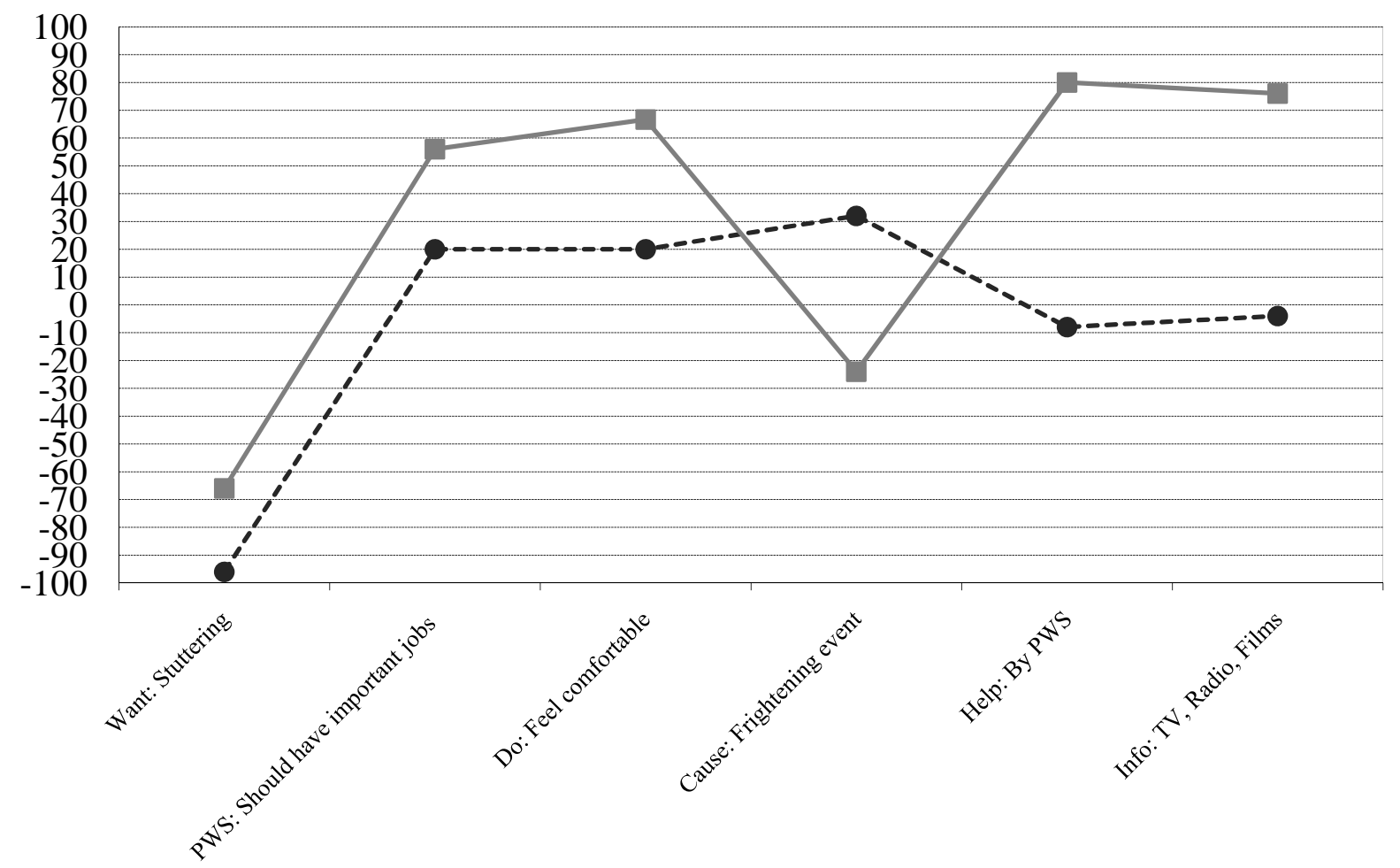

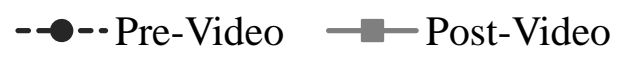

Table 12 shows the largest and smallest PRE versus POST differences for the video presentation for the honors and regular classes. Both classes had comparable differences. The largest difference was, for the honors class, "Help: By PWS” (84) and, for the regular class, “Help: By PWS” (88), which were virtually identical. Most PRE-V versus POST-V means for both classes generally changed in the same direction as well. This can be seen in items, "Want: Stuttering” (-72 vs. -42 [honors], -96 vs. -66 [regular]), “-83 vs. -100 [honors], -44 vs. -88 [regular]) and "PWS: Difficult to listen to" (11 vs. -67 [honors], -20 vs. -40 [regular]. There were exceptions however, “Do: Fill in words” (-67 vs. -89 [honors], -48 vs. -24 [regular]” where the regular class had a stronger undesirable reaction that become even stronger after the video while the honors class had a weaker undesirable reaction beforehand and a positive direction of change after the video. For the item, “Do: Say 'slow down/relax’” (-39 vs. -89 [honors], -16 vs. -12 
[regular]) the regular class had a less desirable reaction when compared to the honors class before the video and had an opposite reaction after the video where they were more likely to tell a PWS to slow down or relax.

\section{Table 12}

Honors and Regular Class Video Presentation

\begin{tabular}{|c|c|c|c|c|c|c|c|}
\hline \multirow[b]{2}{*}{$\begin{array}{l}\text { Questionnaire } \\
\text { Items: }\end{array}$} & \multicolumn{3}{|c|}{ Honors Class } & \multicolumn{3}{|c|}{ Regular Class } & \multirow[b]{2}{*}{$\begin{array}{l}\text { Questionnaire } \\
\text { Items: }\end{array}$} \\
\hline & Pre & Post & Diff & Pre & Post & Diff & \\
\hline Largest Changes & & & & & & & Largest Changes \\
\hline Help: By PWS & -17 & 67 & 84 & -8 & 80 & 88 & Help: By PWS \\
\hline $\begin{array}{l}\text { PWS: Difficult to } \\
\text { listen to }\end{array}$ & 11 & -67 & 78 & -4 & 76 & 80 & $\begin{array}{l}\text { Info: TV, Radio, } \\
\text { Films }\end{array}$ \\
\hline Cause: Genetics & 33 & -44 & 77 & 32 & -24 & 56 & $\begin{array}{l}\text { Cause: } \\
\text { Frightening event }\end{array}$ \\
\hline Smallest Changes & & & & & & & Smallest Changes \\
\hline $\begin{array}{l}\text { Aspects: Speaking } \\
\text { ability }\end{array}$ & 69 & 69 & 0 & 16 & 16 & 0 & $\begin{array}{l}\text { PWS: Shy or } \\
\text { fearful }\end{array}$ \\
\hline $\begin{array}{l}\text { Knowledge: Left } \\
\text { handed }\end{array}$ & -3 & -3 & 0 & 100 & 100 & 0 & $\begin{array}{l}\text { PWS: Can make } \\
\text { friends }\end{array}$ \\
\hline $\begin{array}{l}\text { Knowledge: } \\
\text { Intelligent }\end{array}$ & 31 & 31 & 0 & -64 & -64 & 0 & Concern: Doctor \\
\hline $\begin{array}{l}\text { PWS: Can make } \\
\text { friends }\end{array}$ & 100 & 100 & 0 & -88 & -88 & 0 & $\begin{array}{l}\text { Concern: } \\
\text { Neighbor }\end{array}$ \\
\hline $\begin{array}{l}\text { Cause: Ghosts, } \\
\text { demons, spirits }\end{array}$ & -100 & -100 & 0 & -100 & -100 & 0 & $\begin{array}{l}\text { Do: Joke about } \\
\text { stuttering }\end{array}$ \\
\hline $\begin{array}{l}\text { Info: Magazines, } \\
\text { newspapers, } \\
\text { books }\end{array}$ & -39 & -39 & 0 & 0 & 0 & 0 & Do: Feel pity \\
\hline $\begin{array}{l}\text { Info: Doctors, } \\
\text { nurses, specialists }\end{array}$ & -78 & -78 & 0 & -84 & -84 & 0 & $\begin{array}{l}\text { Cause: Ghosts, } \\
\text { demons, spirits }\end{array}$ \\
\hline $\begin{array}{l}\text { Social: Friends } \\
\text { with PWS }\end{array}$ & 100 & 100 & 0 & -48 & -48 & 0 & $\begin{array}{l}\text { Cause: act of } \\
\text { God }\end{array}$ \\
\hline
\end{tabular}




\section{Post-Video Versus Post-Video + Live}

This section compares the individual class data for after the video presentation versus after the shortened live presentation for each class type. A total of seven statistically significant changes occurred between the honors and regular classes, one and six respectively. The one statistically significant change for the honors class was, “Cause: Genetics” (-44 vs. 44). Even though the regular class had six more statistically significant changes, several of the honors class POST-V+L scores had a greater degree of positive changes with those same items. "PWS:

Nervous or excitable” (0 vs. -44 [honors], 36 vs. -32 [regular]), “Cause: Trying to talk, think too fast” (-44 vs. $-72,20$ vs. -32), “Cause: Frightening event” (-61 vs. $-78,-24$ vs. -64$)$, and “Cause: Learning or habits” (-67 vs. $72,-24$ vs. -64) all show that despite the regular class having a higher number of statistically significant changes the honors class POST-V+L scores resulted in a more positive change. Figure 19 shows the six statistically significant changes for the POST-V versus POST-V+L for the regular class. 
Figure 19. Regular class post-video versus post-video + live presentations

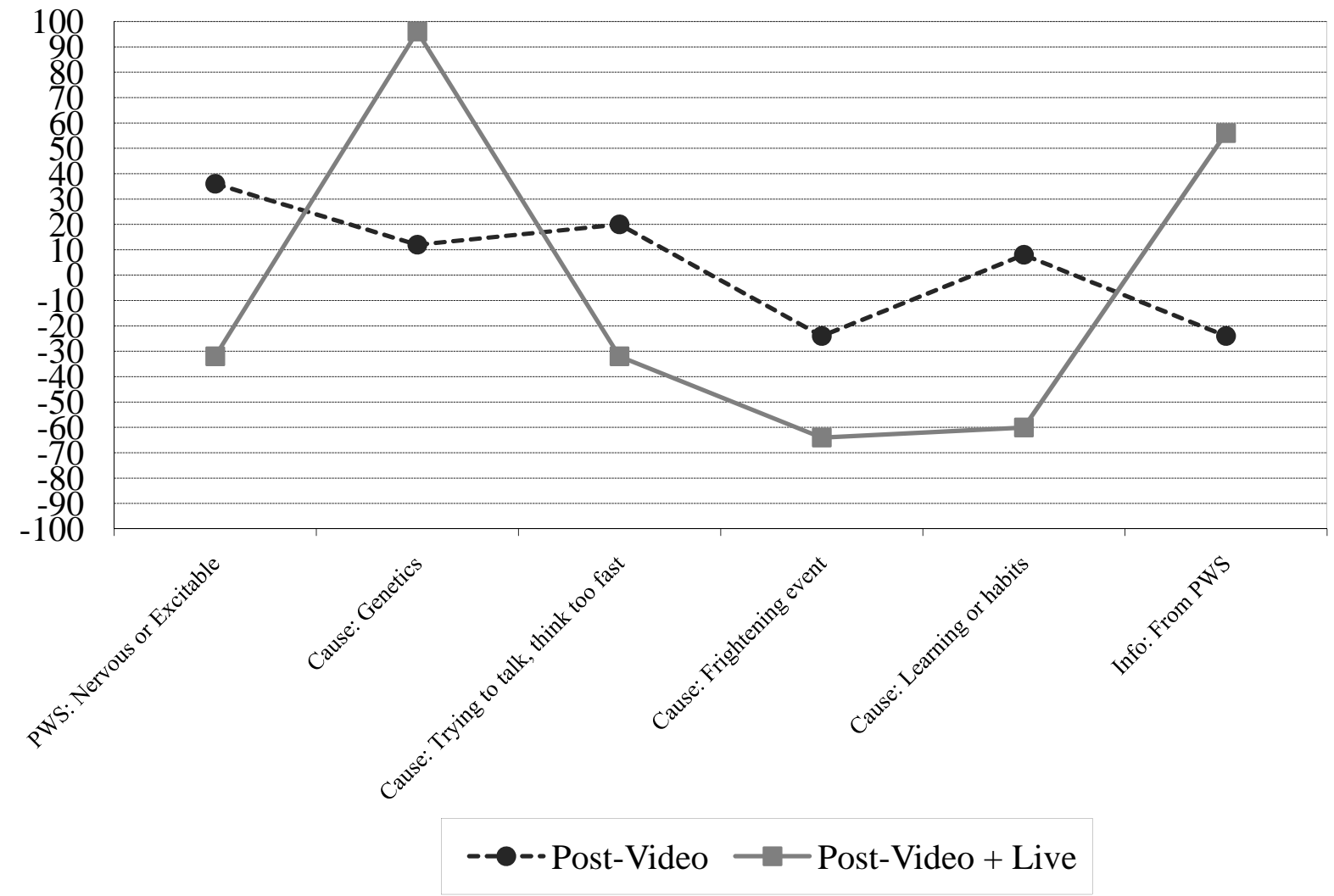

Table 13 shows the largest and smallest POST-V versus POST-V+L mean differences for the honors and regular classes. The largest and smallest differences remain approximately the same between the honors and regular classes. 
Table 13

Honors and Regular Classes Post-Video Vs. Post-Video + Live

\begin{tabular}{|c|c|c|c|c|c|c|c|}
\hline \multirow[b]{2}{*}{$\begin{array}{l}\text { Questionnaire } \\
\text { Items: }\end{array}$} & \multicolumn{3}{|c|}{ Honors Class } & \multicolumn{3}{|c|}{ Regular Class } & \multirow[b]{2}{*}{$\begin{array}{l}\text { Questionnaire } \\
\text { Items: }\end{array}$} \\
\hline & Post & $\begin{array}{l}\text { Post } \\
\text { V+L }\end{array}$ & Diff & Post & $\begin{array}{l}\text { Post } \\
\text { V+L }\end{array}$ & Diff & \\
\hline Largest Changes & & & & & & & Largest Changes \\
\hline Cause: Genetics & -44 & 44 & 88 & 12 & 96 & 84 & Cause: Genetics \\
\hline Info: From PWS & -17 & 33 & 50 & -24 & 56 & 80 & Info: From PWS \\
\hline $\begin{array}{l}\text { PWS: Nervous } \\
\text { or Excitable }\end{array}$ & 0 & -44 & 44 & 36 & -32 & 68 & $\begin{array}{l}\text { PWS: Nervous } \\
\text { or Excitable }\end{array}$ \\
\hline Smallest & & & & & & & Smallest \\
\hline Changes & & & & & & & Changes \\
\hline $\begin{array}{l}\text { Cause: Ghosts, } \\
\text { demons, spirits }\end{array}$ & -100 & -100 & 0 & 30 & 30 & 0 & $\begin{array}{l}\text { Knowledge: } \\
\text { Intelligent }\end{array}$ \\
\hline $\begin{array}{l}\text { Cause: } \\
\text { Mental/Physical } \\
\text { Abuse }\end{array}$ & -67 & -67 & 0 & 100 & 100 & 0 & $\begin{array}{l}\text { PWS: Can make } \\
\text { friends }\end{array}$ \\
\hline $\begin{array}{l}\text { Cause: Virus or } \\
\text { disease }\end{array}$ & -100 & -100 & 0 & 88 & 88 & 0 & $\begin{array}{l}\text { PWS: Can do } \\
\text { any job they } \\
\text { want }\end{array}$ \\
\hline $\begin{array}{l}\text { Info: TV, Radio, } \\
\text { Films }\end{array}$ & 78 & 78 & 0 & -100 & -100 & 0 & $\begin{array}{l}\text { Do: Joke about } \\
\text { stuttering }\end{array}$ \\
\hline $\begin{array}{l}\text { Info: Magazines, } \\
\text { newspapers, } \\
\text { books }\end{array}$ & -39 & -39 & 0 & -76 & -76 & 0 & $\begin{array}{l}\text { Do: Feel } \\
\text { impatient }\end{array}$ \\
\hline $\begin{array}{l}\text { Social: Talk to } \\
\text { PWS }\end{array}$ & 100 & 100 & 0 & 80 & 80 & 0 & Help: By PWS \\
\hline $\begin{array}{l}\text { Social: Friends } \\
\text { with PWS }\end{array}$ & 100 & 100 & 0 & 100 & 100 & 0 & $\begin{array}{l}\text { Social: Talk to } \\
\text { PWS }\end{array}$ \\
\hline
\end{tabular}

Pre-Video Versus Post-Video + Live

A total of 19 statistically significant changes are observed between the honors and regular classes, five and 14, respectively. The honors class continued to show a higher degree of positive change in POST scores than the regular class, despite the large difference in the number of statistically significant changes. Figure 20 and 21 show the statistically significant changes for 
both the honors and regular classes for PRE-V versus POST-V+L. Table 14 shows the largest and smallest mean differences for honors and regular classes for PRE-V versus POST-V+L.

Figure 20. Honors class pre-video versus post-video + live presentations

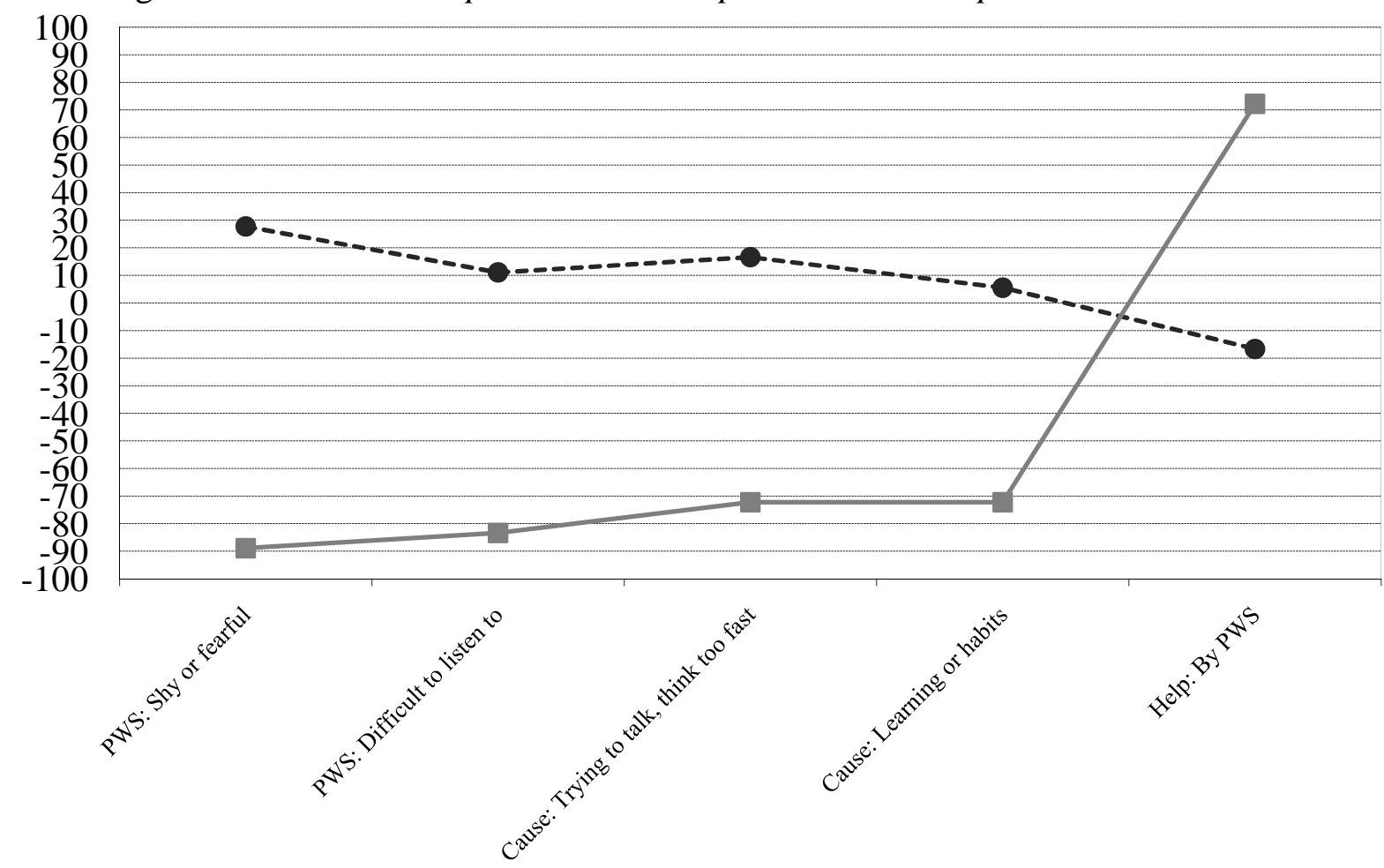

- ๑-- Pre-Video $\rightarrow$ - Post-Video + Live 
Figure 21. Regular class pre-video versus post-video + live presentations

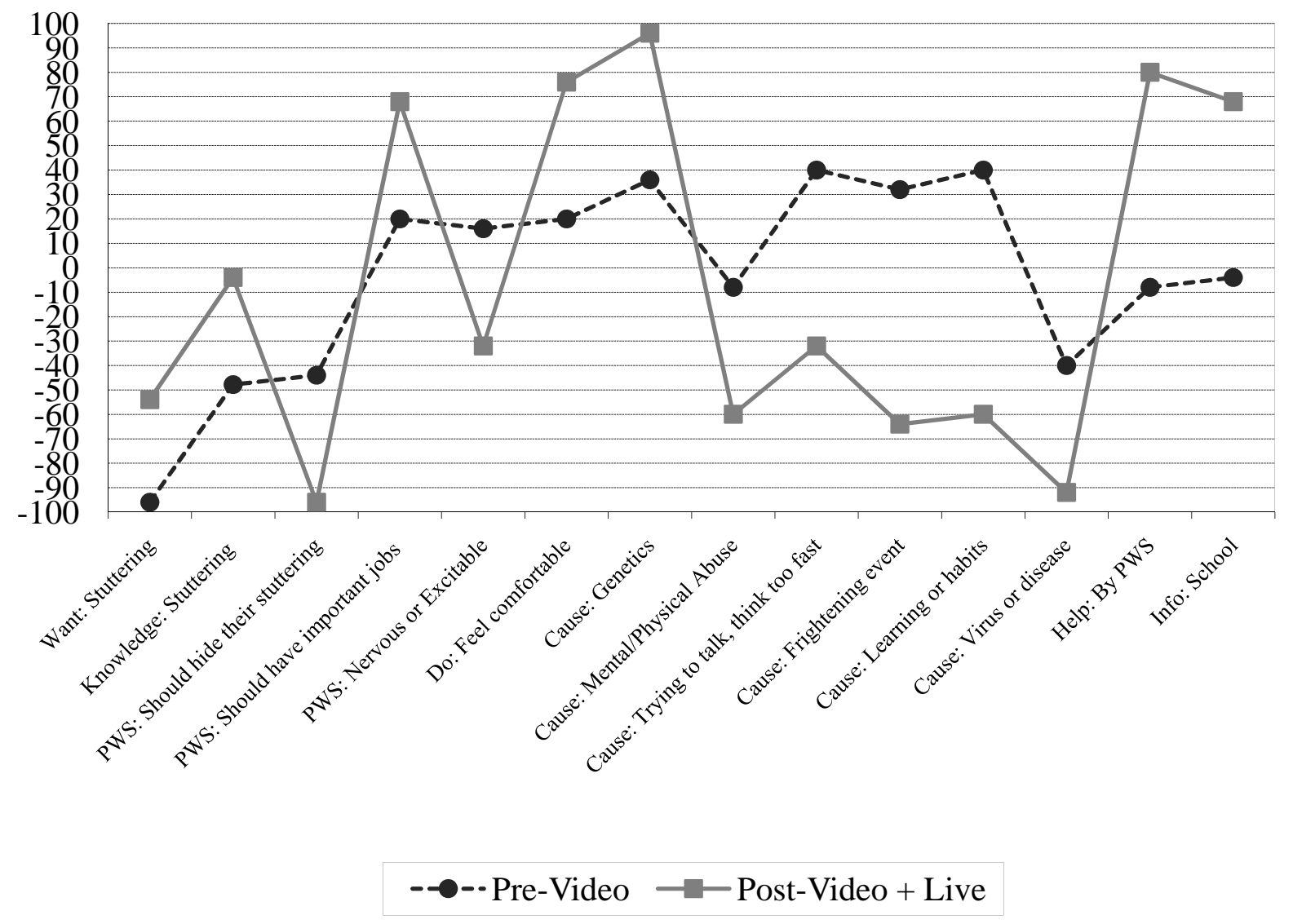


Table 14

Honors and Regular Classes Pre-Video Vs. Post-Video + Live

\begin{tabular}{|c|c|c|c|c|c|c|c|}
\hline \multirow[b]{2}{*}{$\begin{array}{l}\text { Questionnaire } \\
\text { Items: } \\
\text { Largest Changes }\end{array}$} & \multicolumn{3}{|c|}{ Honors Class } & \multicolumn{3}{|c|}{ Regular Class } & \multirow[b]{2}{*}{$\begin{array}{l}\text { Questionnaire } \\
\text { Items: } \\
\text { Largest Changes }\end{array}$} \\
\hline & $\begin{array}{l}\text { Pre } \\
\text { Vid }\end{array}$ & $\begin{array}{l}\text { Post } \\
\text { V }+\mathbf{L}\end{array}$ & Diff & $\begin{array}{l}\text { Pre } \\
\text { Vid }\end{array}$ & $\begin{array}{l}\text { Post } \\
\text { V+L }\end{array}$ & Diff & \\
\hline $\begin{array}{l}\text { PWS: Difficult to } \\
\text { listen to }\end{array}$ & 11 & -83 & 94 & 40 & -60 & 100 & $\begin{array}{l}\text { Cause: Learning } \\
\text { or habits }\end{array}$ \\
\hline $\begin{array}{l}\text { Cause: Trying to } \\
\text { talk, think too fast }\end{array}$ & 17 & -72 & 89 & 32 & -64 & 96 & $\begin{array}{l}\text { Cause: } \\
\text { Frightening event }\end{array}$ \\
\hline Help: By PWS & -17 & 72 & 89 & -8 & 80 & 88 & Help: By PWS \\
\hline Smallest Changes & & & & & & & Smallest Changes \\
\hline $\begin{array}{l}\text { Impression: } \\
\text { Mentally ill }\end{array}$ & -6 & -6 & 0 & -- & -- & -- & -- \\
\hline Want: Obese & -97 & -97 & 0 & -- & -- & -- & -- \\
\hline $\begin{array}{l}\text { Knowledge: } \\
\text { Intelligent }\end{array}$ & 31 & 31 & 0 & -- & -- & -- & -- \\
\hline $\begin{array}{l}\text { PWS: Can make } \\
\text { friends }\end{array}$ & 100 & 100 & 0 & -- & -- & -- & -- \\
\hline $\begin{array}{l}\text { PWS: Lead } \\
\text { normal lives }\end{array}$ & 100 & 100 & 0 & -- & -- & -- & -- \\
\hline $\begin{array}{l}\text { Concern: Brother } \\
\text { or sister }\end{array}$ & -72 & -72 & 0 & -- & -- & -- & -- \\
\hline $\begin{array}{l}\text { Cause: Ghosts, } \\
\text { demons, spirits }\end{array}$ & -100 & -100 & 0 & -24 & -24 & 0 & $\begin{array}{l}\text { Knowledge: } \\
\text { Mentally ill }\end{array}$ \\
\hline $\begin{array}{l}\text { Info: Personal } \\
\text { Experience }\end{array}$ & -56 & -56 & 0 & 100 & 100 & 0 & $\begin{array}{l}\text { PWS: Can make } \\
\text { friends }\end{array}$ \\
\hline $\begin{array}{l}\text { Info: Magazines, } \\
\text { newspapers, } \\
\text { books }\end{array}$ & -39 & -39 & 0 & 96 & 96 & 0 & $\begin{array}{l}\text { PWS: Lead } \\
\text { normal lives }\end{array}$ \\
\hline Info: Internet & -67 & -67 & 0 & -100 & -100 & 0 & $\begin{array}{l}\text { Do: Joke about } \\
\text { stuttering }\end{array}$ \\
\hline $\begin{array}{l}\text { Social: Friends } \\
\text { with PWS }\end{array}$ & 100 & 100 & 0 & -48 & -48 & 0 & Do: Fill in words \\
\hline
\end{tabular}

-- refers to an uneven number of identical smallest differences between honors and regular classes for POST-V vs. POST-V+L 


\section{Pilot Study Comparison}

The pilot study, summarized earlier, was conducted with the same purpose as this investigation, i.e., to positively influence adolescent's attitudes towards stuttering. This section compares the current study with that pilot study. In order to compare the two studies, the data for the pilot study were converted. In the version of the POSHA-E used in the pilot study, all questionnaire items requested respondent ratings in a scale of 1 to 9 , with a choice for “I don't know.” As explained above, the general section in the current adaptation of the POSHA-E requested scale ratings of 1 to 5 ; the detailed section requested categorical judgments of "yes," "no," and "not sure," that were converted to numbers from 1 to 3 . As displayed throughout the results section, all scaled items of the pilot study were similarly converted to a -100 to +100 scale, where -100 represents the most negative, 0 represents unsure or neutral, and +100 represents the most positive rating. Only 45 questionnaire items that were identical between the two POSHA-E questionnaires were compared.

On the earlier version of the POSHA-E during the pilot study, the etiology section had two separate questionnaire items relating to abuse, i.e., "I believe stuttering is caused by physical abuse" and "I believe stuttering is caused by emotional abuse." These two items were combined into one for the current version of the POSHA-E to state, "I believe stuttering is caused by being mentally or physically abused.” All data on the earlier version of the POSHA-E for these two items were combined into one rating by taking the mean of the two ratings.

\section{Combined Data Comparison}

Overall data comparison can be seen between the pilot and current studies in Figures 22, 23 and 24. Each Figure shows a portion of questionnaire items for PRE-L versus POST-L. PRE- 
L and POST-L scores for a majority of items in both studies followed the same trends for the comparable 45 items, "PWS: Nervous or excitable” (0 vs. -44 [pilot], 28 vs. -70 [current]), "PWS: Shy or fearful” (-5 vs. $-36,15$ vs. -65$)$ and “Do: Feel impatient” (-35 vs. $-45,-63$ vs. -90$)$. There were a few exceptions, e.g., “Concern: Neighbor” (-43 vs. -11, -80 vs. -90), “Do: Fill in their words" (-50 vs. $-34,-30$ vs. -85 ) and "Want: Stuttering” (-59 vs. $-61,-76$ vs. -43$)$. In these cases, the pilot and current studies had opposite directions of change. In the current study, PRE-L versus POST-L showed greater positive changes in a majority of items as well, e.g., "People who stutter are nervous or excitable” (0 vs. -44 [pilot], 28 vs. - -70 [current]), "People who stutter are shy or fearful" (-5 vs. -36, 15 vs. -65) and "I believe stuttering is caused by a virus or disease" (-33 vs. $-58,-49$ vs. -97). Table 15 shows that the current study had the largest differences between PRE and POST means as well. The pilot and current study shared the largest difference for the item, "Info: From PWS” (62 [pilot] vs. 81 [current]). 
Figure 22. General items pilot Study versus current study

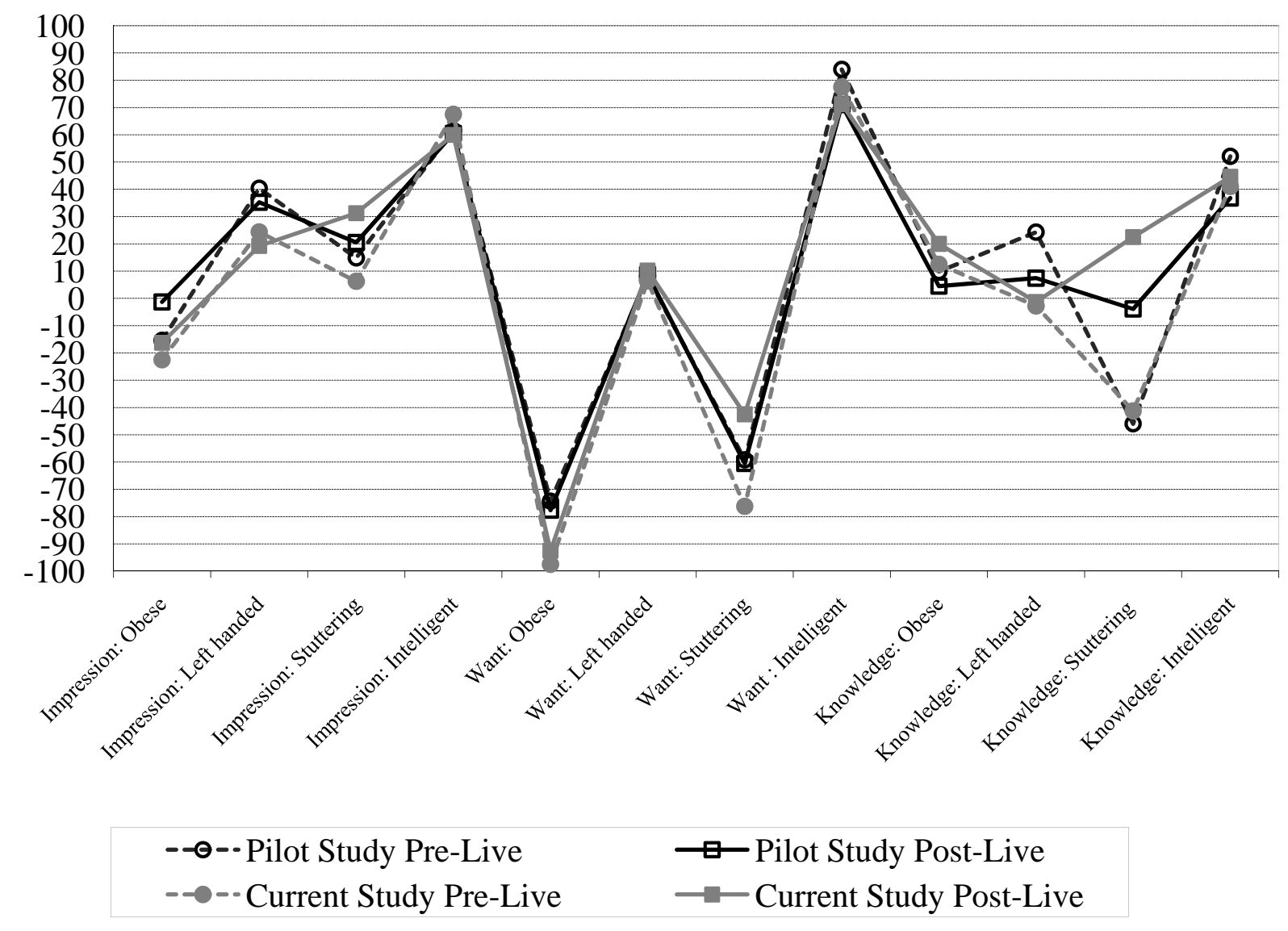


Figure 23. Detailed items pilot study versus current study

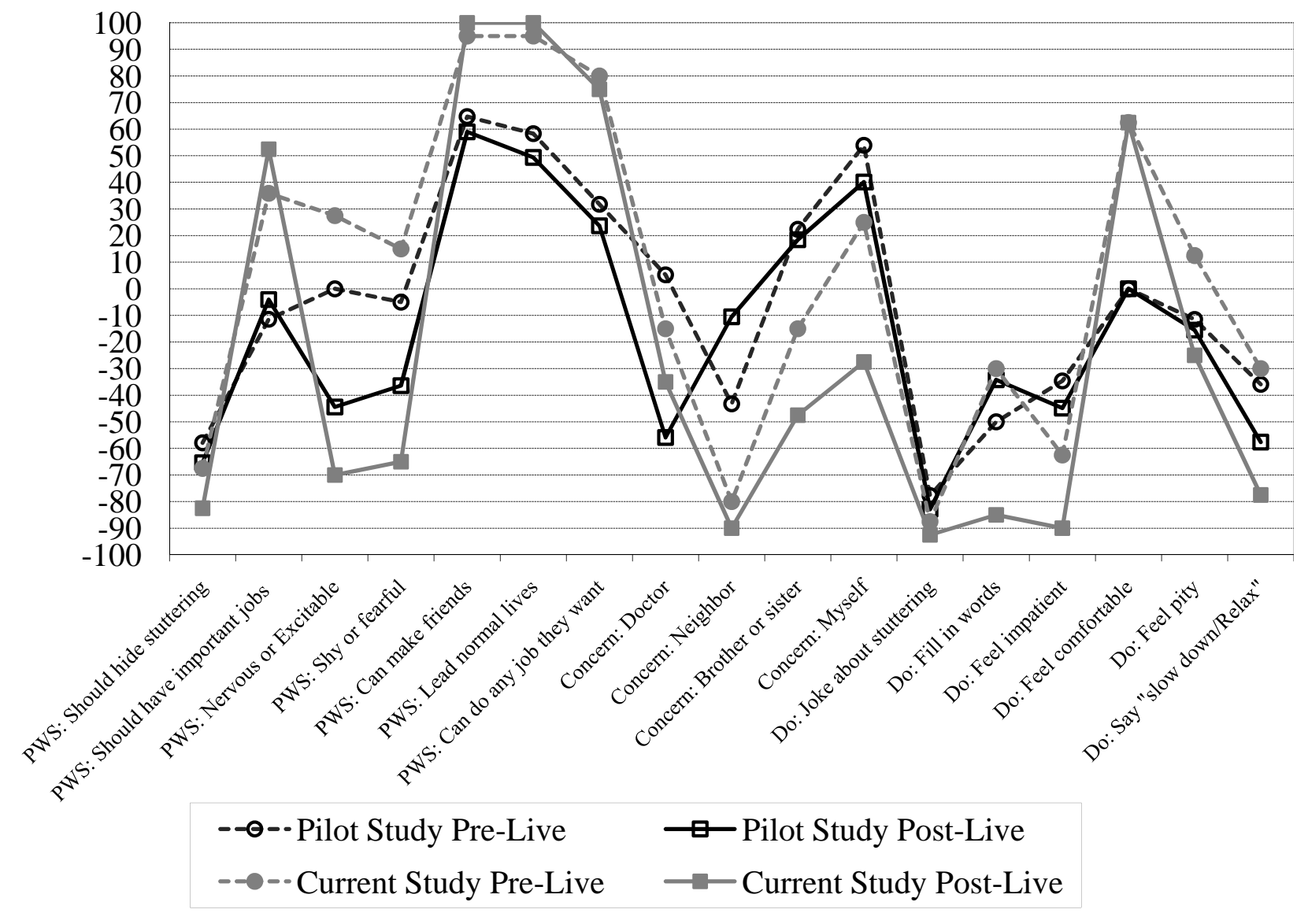


Figure 24. Detailed items pilot study versus current study

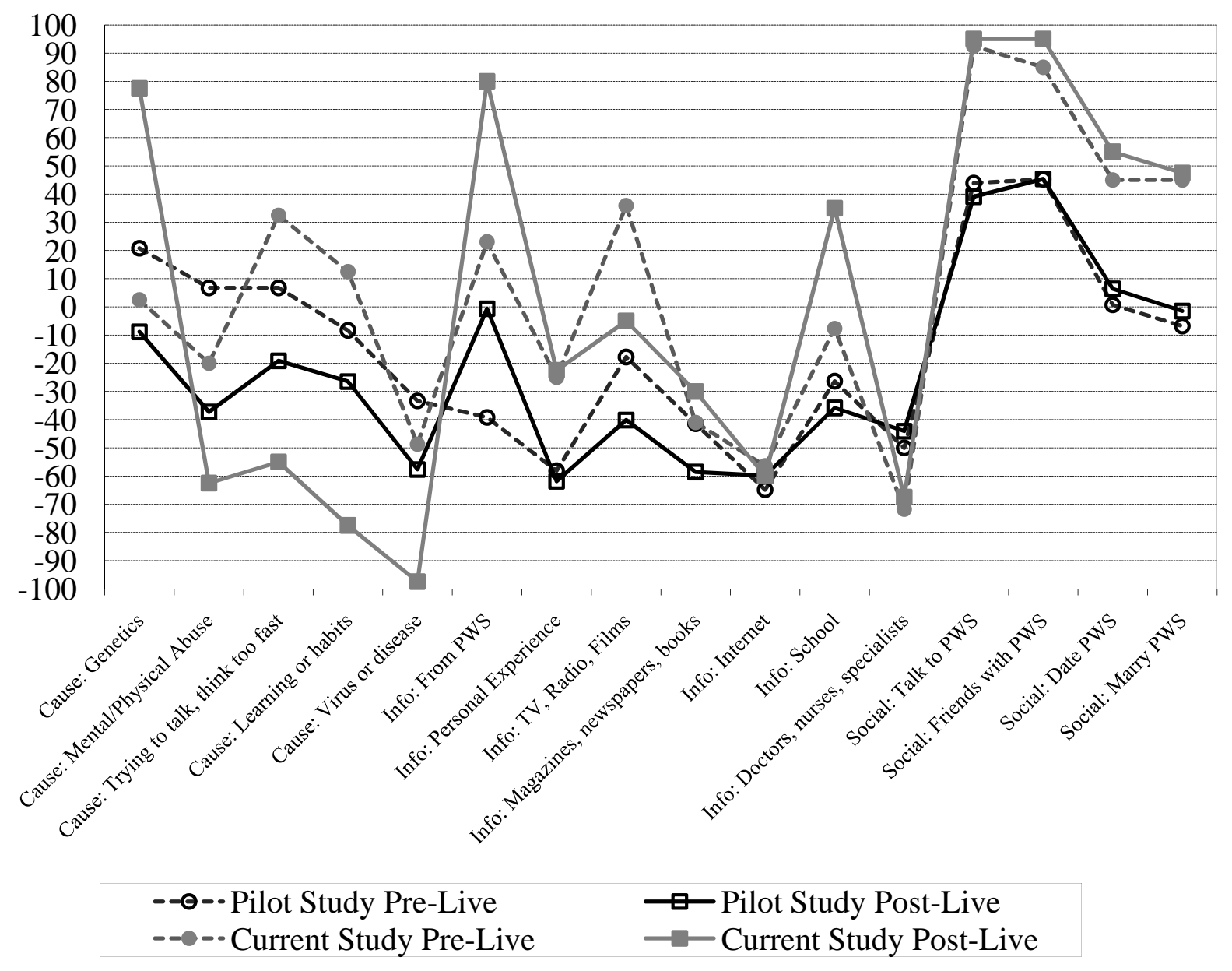


Table 15

Pilot Study vs. Current Study Live Presentations

\begin{tabular}{|c|c|c|c|c|c|c|c|}
\hline $\begin{array}{l}\text { Questionnaire } \\
\text { Items: }\end{array}$ & $\begin{array}{l}\text { Pre } \\
\text { Pilot } \\
\end{array}$ & $\begin{array}{c}\text { Pre } \\
\text { Current } \\
\end{array}$ & Diff & $\begin{array}{l}\text { Post } \\
\text { Pilot }\end{array}$ & $\begin{array}{c}\text { Post } \\
\text { Current } \\
\end{array}$ & Diff & $\begin{array}{l}\text { Questionnaire } \\
\text { Items: }\end{array}$ \\
\hline Largest Changes & & & & & & & Largest Changes \\
\hline $\begin{array}{l}\text { Do: Feel } \\
\text { comfortable }\end{array}$ & 0 & 63 & 63 & -9 & 78 & 86 & Cause: Genetics \\
\hline Info: From PWS & -39 & 23 & 62 & -1 & 80 & 81 & Info: From PWS \\
\hline $\begin{array}{l}\text { Info: TV, Radio, } \\
\text { Films }\end{array}$ & -18 & 36 & 54 & -11 & -90 & 79 & $\begin{array}{l}\text { Concern: } \\
\text { Neighbor }\end{array}$ \\
\hline Smallest Changes & & & & & & & Smallest Changes \\
\hline $\begin{array}{l}\text { Knowledge: } \\
\text { Obese }\end{array}$ & 10 & 13 & 3 & 61 & 60 & 1 & $\begin{array}{l}\text { Impression: } \\
\text { Intelligent }\end{array}$ \\
\hline $\begin{array}{l}\text { Want: Left } \\
\text { handed }\end{array}$ & 8 & 6 & 2 & 71 & 71 & 0 & Want : Intelligent \\
\hline $\begin{array}{l}\text { Info: Magazines, } \\
\text { newspapers, } \\
\text { books }\end{array}$ & -41 & -41 & 0 & -60 & -60 & 0 & Info: Internet \\
\hline
\end{tabular}

\section{Respondent Evaluations}

After hearing and watching the live, video, and shortened live presentations, all participants were asked to write comments about the presentations and then to rate each presentation on a scale from 1 to 9 (“very poor” to “excellent”). All comments are listed verbatim in Appendix 10. Mean ratings were positive after both treatments, but were approximately 1 scale value higher for the live presentations than the video for both the honors and regular classes. The presentations ratings are shown in Table 16. 
Table 16

Presentation Ratings

\begin{tabular}{lccc}
\hline & $\begin{array}{c}\text { Combined } \\
\text { Classes }\end{array}$ & Regular Class & Honors Class \\
\hline $\begin{array}{l}\text { Live } \\
\text { Presentation: }\end{array}$ & 8.68 & 8.50 & 8.85 \\
$\begin{array}{l}\text { Video } \\
\text { Presentation: }\end{array}$ & 7.55 & 7.38 & 7.72 \\
$\begin{array}{l}\text { Short Live } \\
\text { Presentation: }\end{array}$ & 8.56 & 8.28 & 8.83 \\
\hline
\end{tabular}

The presenter's stuttering severity was also rated on a scale of 1 to 9 after all presentations. The stuttering severity rating data are shown in Table 17. Whereas regular and honors classes were inconsistent relative to the type of presentation, combined data indicated that the author's stuttering was perceived as less severe after the live presentation than either the video or the video plus live presentation by more than 1.5 scale values.

Table 17

Stuttering Severity Ratings

\begin{tabular}{lccc}
\hline & $\begin{array}{c}\text { Combined } \\
\text { Classes }\end{array}$ & Regular Class & Honors Class \\
\hline $\begin{array}{l}\text { Live } \\
\begin{array}{l}\text { Presentation: } \\
\text { Video }\end{array}\end{array}$ & 5.30 & 5.80 & 4.80 \\
$\begin{array}{l}\text { Presentation: } \\
\text { Short Live } \\
\text { Presentation: }\end{array}$ & 6.92 & 6.74 & 7.11 \\
\hline
\end{tabular}

Also, at the end of the post questionnaires, participants were asked after the video and live presentations to indicate whether or not they thought the author's stuttering was faked. Results can be seen in Table 18. Sixty-five to $70 \%$ indicated that they believed the stuttering to 
be faked after the live presentations, compared to only 0-5\% agreed that stuttering was not real after they watched the True Life video.

Table 18

Participants Who Thought Stuttering Was Faked

\begin{tabular}{lccc}
\hline & $\begin{array}{c}\text { Combined } \\
\text { Classes }\end{array}$ & Regular Class & Honors Class \\
\hline $\begin{array}{l}\text { Live } \\
\text { Presentation: }\end{array}$ & $70 \%(35 / 50)$ & $73 \%(22 / 30)$ & $65 \%(13 / 20)$ \\
$\begin{array}{l}\text { Video } \\
\text { Presentation: }\end{array}$ & $4.6 \%(2 / 43)$ & $4 \%(1 / 25)$ & $5 \%(1 / 18)$ \\
$\begin{array}{l}\text { Short Live } \\
\text { Presentation: }\end{array}$ & $2.3 \%(1 / 43)$ & $0 \%(0 / 25)$ & $5 \%(1 / 18)$ \\
\hline
\end{tabular}

Also, at the end of the post questionnaires, participants were asked if they had heard the presenter speak before, or seen the video entitled, True Life: I Stutter before. Responses for these questions can be seen in Table 19. Fifty-one percent of participants within the regular classes and $26 \%$ within the honors classes heard the presenter speak before. Similarly, $60 \%$ of participants within the regular class and $27.7 \%$ within the honors class watched the video before.

Table 19

Additional POSHA-E Questions

\begin{tabular}{lccc}
\hline & $\begin{array}{c}\text { Combined } \\
\text { Classes }\end{array}$ & $\begin{array}{c}\text { Regular } \\
\text { Classes }\end{array}$ & $\begin{array}{c}\text { Honors } \\
\text { Classes }\end{array}$ \\
\hline Have you heard the presenter speak to & $39.8 \%$ & $51.1 \%$ & $26.3 \%$ \\
you before? & $(33 / 83)$ & $(23 / 45)$ & $(10 / 38)$ \\
Have you seen the documentary video & $* 46.5 \%$ & $* 60 \%$ & $* 27.7 \%$ \\
entitled, True Life: I Stutter, before? & $(20 / 43)$ & $(15 / 25)$ & $(5 / 18)$ \\
\hline * indicates that percents represent data from video classes only (honors video and \\
regular video)
\end{tabular}




\section{DISCUSSION}

POSHA-E Results

POSHA-E results suggested that adolescents possessed stereotypes and negative attitudes towards stuttering. Further these stereotypes and negative attitudes were prevalent across gender and class type. The stuttering stereotype has been described most commonly when one assigns personality traits to PWS, as can be seen in questionnaire items, "People who stutter are nervous or excitable" and "People who stutter are shy or fearful." The data supports the hypothesis that high school students indeed manifest the so-called "stuttering stereotype" (Evans, Healey, Kawai \& Rowland, 2008; Kirsch, 2007).

Items such as, "People who stutter should try to hide their stuttering," "If I were talking to a person who stutters I would fill in their words," and "If I were talking to a person who stutters I would feel impatient” all make reference to negative attitudes or reactions towards stuttering but were not regarded here as evidence of the stuttering stereotype. Also, a general lack of knowledge of stuttering of the etiology of stuttering was observed, in that the cause of stuttering rated the highest amongst PRE items was, "I believe stuttering is caused by trying to think or talk too fast." One can hypothesize that this general lack of knowledge could lead to adolescents forming incorrect ideas about the cause of stuttering and thereby contribute to negative or unhelpful beliefs and reactions.

\section{Pre-Treatment Comparison}

The data from the present study demonstrated negative reactions or stereotypes towards people who stutter, but varied depending on class type or gender. In comparing groups prior to treatment, honors and regular classes were characterized by statistically significant differences. 
The honors classes generally had more positive attitudes towards stuttering for most items compared to the regular classes. The current study adds support to the pilot study finding that honors classes had more positive attitudes toward stuttering than regular students. Accordingly, the hypothesis that honors students would have more positive attitudes before either treatment than regular class students is supported. By contrast, male and female ratings manifested no significant differences. As the bulk of the literature reviewed suggests (Evans et al., 2008; Hartford \& Leahy, 2007; Langevin \& Hagler, 2004; Patterson \& Pring 1991), the hypothesis that no differences between males and females would occur was supported. Predictably, and providing evidence of unbiased treatment grouping, the pre-live presentation versus pre-video presentation groups were very similar.

\section{Live Presentations Versus Video Presentations (Combined Classes)}

After listening to the live presentation, contrary to three of the four studies attempting to change public opinion (Leahy, 1994; McGee et al., 1996; Snyder, 2001), respondents’ ratings indicated numerous positive attitude changes towards people who stutter. Results from this study do not support Snyder’s (2001) notion that, “It may have been naïve to believe that high school student's perceptions of individuals who stutter could be altered...” (p. 244). It should be noted that seven of eight listed causes of stuttering on the POSHA-E were incorrect in general (e.g. learning or habits), and one was factual (e.g. genetic inheritance). Six of the seven incorrect (negative) beliefs decreased considerably, and five of these were statistically significant. The other negative questionnaire item, "I believe stuttering is caused by ghosts, demons or spirits" had PRE-L and POST-L scores of -100. Of 62 total questionnaire items, a large majority, 69\% (43/62), changed after the presentations in a positive direction; $10 \%$ (6/62) changed in a negative 
direction, 3\% (2/62) had no change, and 18\% (11/62) were ambiguous, as shown in Appendix 4. The reader will recall that questionnaire items that were ambiguous were neither positive nor negative in their direction of change, such as, "My knowledge about stuttering comes from doctors, nurses, or other specialists.” Out of the six questionnaire items that changed in a negative direction, four had no relation to stuttering. These questions included, "My overall impression of someone who is left handed," "My overall impression of someone who is mentally ill," "My overall impression of someone who is intelligent," and "I would want to be someone who is left handed.” The remaining negative changes were, "People who stutter can do any job they want” ( 80 vs.75) and "I believe stuttering should be helped by a speech and language pathologist” (88 vs. 83). The magnitude of negative changes in these latter two items was small and insignificant.

Several positive attitude changes also occurred after watching the video presentation, but these were not as dramatic as from the live presentation. One statistically significant negative attitude change occurred for the item, "I believe stuttering is caused by genetic inheritance” (35 vs. -12). While this is a negative change, since all items in the etiology section reduced from PRE-V to POST-V, as discussed in the results, the possibility exists that participants were less aware of what the actual cause of stuttering is, due to a lack of information about etiology.

The shortened live presentation that followed the video presentation had an added positive influence on the students' attitudes. Certain items that either changed in a negative direction, or were unchanged after watching the video presentation, were altered positively after hearing the shortened live presentation. For the item, "People who stutter are nervous or excitable” (19 [pre-video] vs. 21 [post-video] vs. -37 [post V+L]), the video presentation had little to no effect, while listening to the author in person had a statistically significant effect. The 
item, “I believe stuttering is caused by genetic inheritance” (35 [pre-video] vs. -12 [post-video] vs. 74 [post $\mathrm{V}+\mathrm{L}]$, changed statistically significantly only after the shortened live presentation. The shortened live presentation for a majority of items either reversed a negative impact of the video presentation, i.e. "I believe stuttering is caused by genetic inheritance” (-12 [post-video] vs. 74 [post-V+L] or further influenced attitudes in a positive direction, i.e. "People who stutter are difficult to listen too" (-7 [pre-video] vs. -51 [post-video] vs. -73 [post-V+L]).

Most of the questionnaire items that showed statistically significant changes related directly to the information presented in either the live presentation or video presentation. For example, in the live presentation, the presenter included a section about the etiology of stuttering. He pointed out that stuttering has a genetic component and that stuttering is not caused by abuse and some other the options listed in the etiology section. POST-L reflected this directly in that, after the live presentation, one of the largest changes was observed for, "I believe stuttering is caused by genetic inheritance” (2 vs. 78) ( $<$ 0.0005). During the video presentation, participants observed the author trying to get a job as a bartender and eventually being hired. POST-V also appeared to reflect this job search in the item, "People who stutter should have important jobs” (37 vs. 70).

Interestingly, while POST scores directly reflect the information that was provided in both the live and video presentations, positive changes were also noted on items not directly addressed. For example, the live presentation did not discuss filling in words for a person who stutters; yet, this item decreased dramatically to indicate a positive attitude change (-30 vs. -85). After the video presentation, "I believe stuttering is caused by a very frightening event" (12 vs. 40 ) and "I believe stuttering is caused by learning or habits" (26 vs. -23) both decreased significantly despite the fact that the video did not discuss the cause of stuttering. Perhaps, 
simply the exposure to people who stutter can help reduce certain aspects of the stuttering stereotype, as suggested by Klassen (2002).

Of interest in both the live presentation and video presentation data was the item, "I would want to be a person who has a stuttering disorder.” This item changed positively by statistically significant amounts after both the live and video presentations. The item highlights another important consideration in interpreting the results. For the live presentation, the PRE-L versus POST-L scores for this item were -76 vs. -43 . While it may seem odd that someone would “want to be a person who has a stuttering disorder," it is important to realize that the POST-L is still considerably low on a scale of -100 to 100 . It is for this reason that such a difference can be viewed as a positive change.

\section{Honors Versus Regular Classes}

Both class types appeared to be highly receptive to the live presentation and the video presentations, but mirrored the combined data in that the live presentations had a greater positive impact than the video presentations. The honors classes showed more positive attitudes before and after the live presentation for a large number of items, i.e., "People who stutter are nervous or excitable” (20 vs. -85 [honors live], 35 vs. -55 [regular live]) and "People who stutter are shy or fearful” (0 vs. -90, 30 vs. -40). This same trend was also seen before and after the video presentation, “I would want to be a person who has a stuttering disorder” (-72 vs. -42 [honors video], “-96 vs. -66 [regular video]) and "People who stutter should have jobs where they have to correctly understand and decide important things” (61 vs. 89, 20 vs. 56). Students in the honors classes not only demonstrated more positive attitudes before the presentations, but were more likely to positively alter their attitudes afterwards. One reason for this may be that the honors 
classes were about one year older than the regular classes (Table 1). Another reason may be that they were more likely to absorb the messages from the presentations and because they presumably were more mature, treat the information received more objectively than the regular class students.

\section{Male Versus Female}

Male attitudes after the live presentation were quite different than female attitudes. Four items changed significantly for both males and females, and eight additional items changed significantly for females only. Depending on the item, males and females PRE scores varied, e.g., for the item, "PWS are nervous or excitable," males had PRE-L of 43, and females had PRE-L of 19. In this instance, males had a stronger stereotype toward stutterers. Contrasted to the item, "People who stutter are shy or fearful," male ratings for PRE-L were -7 and female ratings for PRE-L were 27. In this instance females had a stronger stereotype about stutterers. The inference to be drawn from these inconsistencies is that neither males nor females had uniformly stronger negative attitudes as a group. More important, perhaps, females were more likely to alter their attitudes about stutterers after the presentations compared to males. Evidence of this can be seen in items such as "People who stutter are shy or fearful” (-7 vs. -57 [males], 27 vs. -69 [females]) and "People who stutter should have important jobs" (46 vs. 50, 31 vs. 54).

Similar inferences emerge from data comparing ratings for males and females after the video presentation. One change was statistically significant for males, while seven were significant for females. Whereas this seems to favor females in their ability to alter their attitudes more than males, a comparison of PRE scores showed an interesting pattern. Six of the seven questionnaire items that were significant for females reflected stronger negative attitudes before 
the video presentation than for males. These items were: "My overall impression of someone who has a stutter disorder” (18 [male pre] vs. -8 [female pre]), “The amount I know about people who have a stuttering disorder” (-44 vs. -50), "People who stutter are difficult to listen too” (-16 vs. 0), "I believe stuttering is caused by trying to think or talk too fast” (11 vs. 46), "I believe that stuttering is caused by learning or habits” (11 vs. 38) and “Help: By PWS” (5 vs. -25). The seventh item was "I would want to be a person who has a stuttering disorder" (-87 vs. -85), wherein PRE scores were virtually the same. Further, while females had stronger negative attitudes for those specific items, their attitudes changed more than the males in four of the seven statistically significant changes, i.e., "I would want to be a person who has a stuttering disorder" (-66 [male post] vs. -48 female [post]), “People who stutter are difficult to listen to” (-21 vs. 75), "I believe the cause of stuttering is trying to think or talk too fast" (11 vs. -21) and "I believe stuttering should be helped by people who stutter” (68 vs.79). This pattern suggests that while females had worse negative attitudes for some, but not all, items, they were more likely to alter their attitudes, and alter them more than males.

The shortened live presentation after the video presentation produced similar results for both male and females. For a majority of questionnaire items, attitudes changed positively for both males and females. In particular, two items improved significantly for males and females, and another item changed for the males only.

\section{Pilot Study}

Not surprisingly, both the pilot and current studies had similar results as seen in Figures 22, 23, and 24. Still there were important differences. Data suggest that the current study was more successful in changing attitudes toward stuttering in high schools students in a positive 
direction. Not only were there a greater number of significant changes, i.e. five in the pilot study versus 13 in the current study, the degree of positive change was greater. This trend is apparent for many items, "People who stutter are nervous or excitable” (0 vs. -44 [pilot], 28 vs. -70 [current]), “The amount I know about people who have stuttering disorders” (-46 vs. $-4,-41$ vs. 45), and "If I were talking to a person who stutters, I would feel impatient" (-35 vs. -45 , -63 vs. 90).

One explanation for differences between the two studies is that, for the current study, the live presentations were more concise and prepared. The author followed a script and a specific order of topics when delivering the live presentations in the current study. A section about the cause of stuttering was added to the live presentation in this study after journal responses from the pilot study revealed that participants wished to learn more about the cause of stuttering. The results of the addition is evident, in that data for the pilot study showed only one statistically significant change for the item, "I believe stuttering is caused by mental or physical abuse" (7 [pre pilot] vs. -37 [post pilot]) and showed a statistically non-significant negative change for "I believe the cause of stuttering is genetic inheritance” (21 vs. -9). By contrast, the current study showed statistically significant positive changes in all items for the etiology section, including genetic inheritance.

\section{Respondent Evaluations}

Respondent evaluations are important in gauging true opinions of participants. Constructive criticism of the presentations can be potentially useful for future attempts to altering negative attitudes about stuttering. With that in mind, adolescents are known to be honest, and at times brutally honest. Such honesty can become useful in selecting specific 
strategies for changing public opinion. As noted, all students were asked to write a journal response after the live presentations and video presentations/shortened live presentations. All of the journal entries are included verbatim in Appendix 10.

All journal responses were complimentary; yet some important insights emerged. One was that many participants believed that the author was not really stuttering. For example, respondents wrote, “I truly enjoyed the speech Tim gave. When he first started talking I didn’t expect that, but who would”; “I really didn’t know whether this man was faking the stutter at first”; "When first hearing Tim Flynn begin to talk, I almost thought he was faking it at first”; "Tim is a very special person. At first I thought his stutter was fake”; and "The speaker really surprised me because I thought he was faking being speech impaired or being a stamee.” Results from asking for students to raise their hands at the end of each live presentation revealed that $65 \%$ (13/20 in the honors class) and 73\% (22/30 in the regular class) believed the author's stuttering was faked when he first began to talk (Table 18). This is consistent with the findings in the pilot study where 57\% (40/70) of participants also believed the author's stuttering was faked. It is not clear why the majority of students believed he had been faking his stutter, but perhaps it was due to the shock one experiences in not expecting someone to stutter. Some participants referred to this shock value, such as: “...Once he started to talk, I was in complete SHOCK! I had no idea that this guy actually was going to be stuttering...” and "Our guest speaker last class, Tim Flynn, was really amazing. To be honest, when Mr. Flynn started talking, his stuttering shocked me...”

With stuttering, there is no warning sign before the stuttering person starts to speak, so listeners are unprepared for such an obvious and overt behavior as moderate to severe stuttering to take place. After the live presentations, while speaking with participants, the author noted that 
many made reference to how his stuttering was intermittent, i.e., severe at one moment and then nonexistent at the next. For those who did not have previous exposure to stuttering, this intermittency of stuttering may have been mistaken for the speaker trying to give an example of stuttering rather than illustrating the normal inconsistency of stuttering.

\section{CONCLUSION}

The current study demonstrates that attitudes towards stuttering can be improved in high school students. The research does not support the previous three studies (Leahy, 1994; McGee, 1996; Snyder, 2001) which indicated that positive attitude changes and reductions in the stuttering stereotype cannot be achieved by providing information about the disorder. Rather, this study shows that there is a direct relationship between how the information is presented and attitude changes. Comments from the journal entries suggest that the participants in this study could relate to the author because he was relatively close to their age. Moreover, his oral presentation consisted generally of personal stories that happened to him during his high school experience, and it was the author's intention that the participants in this study could relate on that basis. The video was a part of a series on MTV called True Life, a television series designed for adolescents. Taken together, the age-appropriate characteristics of the presentation and video in this study suggest that a major key in improving stuttering attitudes may be the extent to which the audience believes they can relate to a stuttering speaker.

This study clearly demonstrated that (a) high school students did show evidence of a stuttering stereotype amongst questionnaire items; (b) a live presentation positively impacted them more than a video presentation; (c) a shortened live presentation further positively impacted their negative attitudes from the video presentation; (d) students in honors classes were 
less biased and showed more positive attitude change than students in regular classes; and (e) whereas female and male students had few differences in attitudes, females were more likely to change their attitudes in a positive direction than males.

Further research should further test live, video, and other forms of advocacy with different age groups and settings. Moreover, since this study dealt only with short-term changes in attitudes, it would be useful as well to replicate these findings and then measure long-term changes in a longitudinal study of attitude changes to determine if these forms of advocacy leave a lasting impression of positive attitude changes towards stuttering. 


\section{REFERENCES}

Betz, I. R., Blood, G.W., \& Blood, I.M. (2007). University students’ perceptions of pre-school and kindergarten children who stutter. Journal of Communication Disorders, 41, 259273.

Blood, G. W. (2000). The stigma of stuttering: Centuries of negative perceptions and stereotypes. Paper presented at the annual convention of the American Speech-Language Hearing Association. Washington, DC.

Blood, G. W., Blood, I. M., Tellis, G. M., \& Gabel, R. M. (2003). A preliminary study of selfesteem, stigma, and disclosure in adolescents who stutter. Journal of Fluency Disorders, 28, 143-159.

Bloodstein, O., Ratner, B. N. (2008). A Handbook on Stuttering (6 ${ }^{\text {th }}$ Edition). Delmar, Thomson Delmar Learning.

Bondarenko, V. (Producer and Director) (1992). Speaking of Courage (Videotape). Canada: Magic Lantern Communications

Bondarenko, V. (Producer and Director) (1992). Voices To Remember (Videotape). Canada: Magic Lantern Communications

Borsel, J. V., Verniers, I., \& Bouvry, S. (1999). Public awareness of stuttering. International Journal of Phoniatrics, Speech Therapy and Communication Pathology, 51, 124-132.

Breslin, J. W. (1989). Breaking away from subtle biases. Negotiation Theory, 55, 219-222.

Burgess, H. (2003). Stereotypes / Characterization frames. In Guy Burgess and Heidi Burgess (Ed.). Beyond Intractability. Conflict Research Consortium, University of Colorado, Boulder. 
Cooper, E. B., \& Cooper, C. S. (1996). Clinician attitudes toward stuttering: Two decades of change. Journal of Fluency Disorders, 21, 119-135.

Craig, A., Hancock, K., Tran, Y., \& Craig, M. (2001). Prevalence and stereotypes of stuttering in the community. In H.-G. Bosshardt, J. S. Yaruss \& H. F. M. Peters (Eds.). Fluency disorders: Theory, research, treatment and self-help. Proceedings of the Third World Congress on Fluency Disorders in Nyborg, Denmark. International Fluency Association, (pp. 588-592).

Craig, A., Tran, C., \& Craig, M. (2003). Stereotypes towards stuttering for those who have never had direct contact with people who stutter. A randomized and stratified study. Perceptual and Motor Skills, 97, 235-245

Crocker, J., Major, B., \& Steele, C. (1998). Social Stigma. In D. T. Gilbert, S. T. Fiske, G. Lindzey (Eds.), Handbook of social psychology (pp. 504-553). New York. Mcgraw-Hill.

Daly, D. A practitioner's view of stuttering. (1988). ASHA, 30(4), 34-35.

DeLoach, C. P. (1989). Gender, career choices and occupational outcomes among college alumni with disabilities. Journal of Applied Rehabilitation Counseling, 20, 8-12.

Doody, I., Kalinowski, J., \& Armson, J. (1993). Stereotypes of stutterers and nonstutterers in three rural communities in Newfoundland. Journal of Fluency Disorders, 18, 363-373.

Evans, D., Healey, C.E., Kawai, N., \& Rowland, S. (2008). Middle school students’ perceptions of a peer who stutters. Journal of Fluency Disorders, 33, 203-219.

Flynn, T. (2007). An Investigation of Adolescent Opinions on Stuttering. Unpublished manuscript. 
Flynn, T. \& St. Louis, K. (2007, November). An investigation of adolescent opinions on stuttering. Poster session presented at American Speech-Language-Hearing Association Convention, Boston MA.

Gabel, R. M., Blood, G. W., Tellis, G. M., \& Althouse, M. T. (2004). Measuring role entrapment of people who stutter. Journal of Fluency Disorders, 29, 27-49.

Goffman, E. (1963). Stigma: Notes on the management of spoiled identity. Englewood Cliffs, NJ: Prentice-Hall.

Guitar, B. (2006). Stuttering: An Integrated Approach to Its Nature and Treatment. Baltimore: Lippincott, Williams \& Wilkins.

Hartford, E., \& Leahy, M. M. (2007). The perceptions of primary school children of a person who stutters. In J. Au-Yeung \& M. M. Leahy (Eds.). Research, treatment, and self-help in fluency disorders: New Horizons. Proceedings of the Fifth World Congress on Fluency Disorders (pp. 223-229).

Heatherton, T. F., Kleck, R, E., Hebl, M. R., \& Hull, J, G. (2000). The social psychology of stigma. New York: The Guilford Press.

Kalinowski, J., Stuart, A., \& Armson, J. (1996). Perceptions of stutterers and nonstutterers during speaking and nonspeaking situations. American Journal of Speech-Language Pathology, 5, 61-67.

Kalinowski, J. S., Armson, J., Stuart, A., \& Lerman, J. W. (1993) Speech clinicians' and the general public's perceptions of self and stutterers. Journal of Speech-Language Pathology and Audiology, 17, 79-85.

Kirsch, D. I. (2007). Adolescent perceptions of their peers who stutter. Dissertation Abstracts International. (UMI No. 3224098). 
Klassen, T. R. (2001). The complexity of attitudes toward people who stutter. In H.-G. Bosshardt, J. S. Yaruss \& H. F. M. Peters (Eds.). Fluency disorders: Theory, research, treatment and self-help. Proceedings of the Third World Congress on Fluency Disorders in Nyborg, Denmark. International Fluency Association, 605-609.

Klassen, T. R. (2002). Social distance and the negative stereotype of people who stutter. Journal of Speech-Language Pathology and Audiology, 26(2), 90-99.

Langevin, M., \& Hagler, P. (2004). Development of a scale to measure peer attitudes towards children who stutter. In A.K. Bothe (Ed.) Evidence-based treatment of stuttering: Empirical issues and clinical implications (pp 139 - 171). Mahwah, NJ: Lawrence Elrbaum Associates, Inc.

Lass, N. J., Ruscello, D. M., Schmitt, J. F., Pannbacker, M. D., Orlando, M. B., Dean, K. A., \& et al. (1992). Teachers' perceptions of stutterers. Language, Speech, and Hearing Services in Schools, 23, 78-81.

Lass, N. J., Ruscello, D. M., Pannbacker, M., Schmitt, J. F., Kiser, A. M., Mussa, A. M. \& et al. (1994). School administrators' perceptions of people who stutter. Language, Speech, and Hearing Services in Schools, 25, 90-93.

Lass, N. J., Ruscello, D. M., Pannbacker, M. D., Schmitt J. F., \& Everly-Myers, D. S. (1989). Speech-language pathologists' perceptions of child and adult female and male stutterers. Journal of Fluency Disorders, 14, 127-134.

Leahy, M. M. (1994). Attempting to ameliorate student therapist' negative stereotype of the stutterer. European Journal of Disorders of Communication, 29(1), 39-49.

Lees, R. M., \& Stewart, T. (2001). Students’ attitudes and beliefs about stuttering. In H.-G. Bosshardt, J. S. Yaruss \& H. F. M. Peters (Eds.). Fluency disorders: Theory, research, 
treatment and self-help. Proceedings of the Third World Congress on Fluency Disorders in Nyborg, Denmark. International Fluency Association, 610-613.

MacKinnon, S. P. \& Hall, S., MacIntyre, P. D. (2007). Origins of the stuttering stereotype: Stereotype formation through anchoring-adjustment. Journal of Fluency Disorders, 32, 297-309.

Maxwell, D. L., \& Satake, E. (2006). Research and statistical methods in communication sciences and disorders. Clifton Park, NY: Thompson Delmar Learning.

Mayo, R., Mayo, C. M., Jenkins, K. C., \& Graves, L. R. (2004). Public knowledge of stuttering: cross-cultural perspectives. Retrieved January $1^{\text {st }}$, 2009, from http://www.speechpathology.com/articles/article_detail.asp?article_id=232

McGee, L., Kalinowski, J. \& Stuart, A. (1996). Effect of a videotape documentary on high school students' perceptions of a high school male who stutterers. Journal of SpeechLanguage Pathology and Audiology, 20, 240-246.

Nichols, K. (1987). Feelings of Western Carolina University students toward stutterers and stuttering. Unpublished manuscript. Cullowhee, NC: Western Carolina University.

Patterson, J., \& Pring, T. (1991). Listeners attitudes to stuttering speakers: No evidence for a gender difference. Journal of Fluency Disorders, 16, 201-205.

Reichel, I., \& St. Louis, K. O. (2004). Effects of emotional intelligence training in graduate fluency disorders courses. In A. Packman, A. Meltzer, \& H. F. M. Peters (Eds.). Proceedings of the 4th World Congress on Fluency Disorders. (pp. 474-481). Nijmegen, The Netherlands: Nijmegen University Press.

Rice, M. \& Kroll, R. (1997). Workplace experiences of people who stutter. Journal of Fluency Disorders, 22, 140. 
Roesti, S., Tellis, G., \& Gabel, R. (2003). Perceptions of African-American middle and high school students about stuttering. In A. Packman, A. Meltzer, \& H. F. M. Peters (Eds.). Proceedings of the 4th World Congress on Fluency Disorders. (pp. 396-405). Nijmegen, The Netherlands: Nijmegen University Press.

Schutte, N.S., \& Malouff, J. M. (1999). Measuring emotional intelligence and related constructs. Lewiston, NY: Edwin Mellen Press.

Shapiro, A. (1999). Everybody belongs: Changing negative attitudes towards classmates with disabilities. New York: Garland Pub.

Shapiro, D. (1999). Stuttering Intervention. Texas: Pro-Ed.

Schneider, J. (Producer/Director). (2007). True Life: I Stutter [Television series episode]. [New York: MTV].

Snyder, G. J. (2001). Exploratory research in the measurement and modification of attitudes toward stuttering. Journal of Fluency Disorders, 26, 149-160.

Specialty Board on Fluency Disorders (n.d.). Retrieved April 30 ${ }^{\text {th }}$, 2009, from http://www.stutteringspecialists.org/index.html

St. Louis, K. O. (2005, Oct. 18). A global project to measure public attitudes about stuttering. The ASHA Leader, 22, 12-13.

St. Louis, K. O., \& Lass, N. J. (1981). A questionnaire of communicative disorders students’ attitudes toward stuttering. Journal of Fluency Disorders, 6, 49-79.

St. Louis, K. O., Lubker, B. B., Yaruss, J. S., Adkins, T. A., \& Pill, J. C. (2008). Development of a prototype questionnaire to survey public attitudes toward stuttering: Principles and methodologies in the first prototype. The Internet Journal of Epidemiology, 5(2). $<$ http://www.ispub.com/ostia/index.php?xmlFilePath=journals/ije/vol5n2/stuttering.xml> 
St. Louis, K. O., Lubker, B. B., Yaruss, J. S., \& Aliveto, E. F. (In press). Development of a prototype questionnaire to survey public attitudes toward stuttering: Reliability of the second prototype. Contemporary Issues in Communication Sciences and Disorders.

St. Louis, K. O., Reichel, I., Yaruss, J. S., \& Lubker, B. B. (In press). Construct and concurrent validity of a prototype questionnaire to survey public attitudes toward stuttering. Journal of Fluency Disorders.

St. Louis, K. O., Tellis, G., Tuanquin, T. C., Wolfenden, R. P., \& Nicholson, R. M. (2004). Selected attitudes toward stuttering: SLP fluency specialists, generalists, and students. Poster presented at the Annual Convention of the American Speech-Language-Hearing Association. Philadelphia, PA.

Stuttering. (n.d.). Merriam-Webster's Medical Dictionary. Retrieved January $1^{\text {st }}, 2009$, from Dictionary.com website: http://dictionary.reference.com/browse/stuttering

Susca, M., \& Healey, C. E. (2001). Perceptions of simulated stuttering and fluency. Journal of Speech, Language, and Hearing Research, 44, 61-72.

Tversky, A., \& Kahneman, D. (1974). Judgment under uncertainty: Heuristics and biases. Science, 185, 1124-1130.

Van Borsel, J., Verniers, I., \& Bouvry, S. (1999). Public awareness of stuttering. Folia Phoniatrica and Logopaedica, 51, 124-132.

Weisel, A., \& Spektor, G. (1998). Attitudes toward own communication and toward stuttering. Journal of Fluency Disorders, 23, 157-172.

White, P. A., \& Collins, S. R. (1984). Stereotype formation by inference: A possible explanation for the “stutterer” stereotype. Journal of Speech and Hearing Research, 27, 567-570. 
Williams, D.F. \& Diaz, C.F. (1999, October 13). The stereotyping of people who stutter: origins, effects, and controls. Speaking Out Magazine.

Woods, C. L., \& Williams, D. E. (1976). Traits attributed to stuttering and normally fluent males. Journal of Speech and Hearing Research, 19, 267-278.

Yairi, E., \& Williams, D. (1970). Speech clinicians’ stereotypes of elementary school boys who stutter. Journal of Communication Disorders, 3, 161-170. 
Appendix 1. Participants’ experience with five attributes.

\begin{tabular}{|c|c|c|c|c|c|c|c|c|c|c|}
\hline & \multicolumn{2}{|c|}{ Honors Live } & \multicolumn{2}{|c|}{ Honors Video } & \multicolumn{2}{|c|}{ Regular Live } & \multicolumn{2}{|c|}{ Regular Video } & \multirow[b]{2}{*}{$\begin{array}{c}\text { Total \% } \\
\text { Male }\end{array}$} & \multirow[b]{2}{*}{$\begin{array}{c}\text { Total \% } \\
\text { Female }\end{array}$} \\
\hline & Male & Female & Male & Female & Male & Female & Male & Female & & \\
\hline Sample Size & 5 & 15 & 4 & 14 & 9 & 11 & 15 & 10 & & \\
\hline \multicolumn{11}{|c|}{$\begin{array}{l}\text { Following are people I have } \\
\text { known who ... }\end{array}$} \\
\hline \multicolumn{11}{|l|}{ Are obese } \\
\hline 1. Nobody & 0 & 2 & 0 & 0 & 0 & 1 & 0 & 1 & 0.0 & 12.0 \\
\hline 2. Acquaintance & 3 & 6 & 3 & 4 & 5 & 6 & 11 & 7 & 66.7 & 54.0 \\
\hline 3. Close Friend & 1 & 6 & 1 & 10 & 5 & 4 & 3 & 3 & 30.3 & 34.0 \\
\hline 4. Relative & 2 & 9 & 2 & 8 & 6 & 4 & 9 & 4 & 57.6 & 46.0 \\
\hline 5. $\mathrm{Me}$ & 0 & 2 & 0 & 1 & 0 & 0 & 0 & 0 & 0.0 & 4.0 \\
\hline 6. Other & 0 & 1 & 0 & 0 & 0 & 2 & 3 & 1 & 9.1 & 8.0 \\
\hline \multicolumn{11}{|l|}{ Are left handed } \\
\hline 1. Nobody & 0 & 1 & 0 & 5 & 0 & 0 & 0 & 0 & 0.0 & 2.0 \\
\hline 2. Acquaintance & 1 & 3 & 1 & 2 & 4 & 3 & 7 & 5 & 39.4 & 30.0 \\
\hline 3. Close Friend & 4 & 11 & 3 & 3 & 5 & 5 & 13 & 4 & 75.8 & 60.0 \\
\hline 4. Relative & 1 & 7 & 0 & 2 & 5 & 5 & 9 & 4 & 45.5 & 48.0 \\
\hline 5. $\mathrm{Me}$ & 0 & 1 & 1 & 0 & 3 & 1 & 2 & 0 & 18.2 & 6.0 \\
\hline 6. Other & 0 & 0 & 0 & 2 & 1 & 1 & 3 & 2 & 12.1 & 6.0 \\
\hline \multicolumn{11}{|c|}{ have stuttering disorders } \\
\hline 1. Nobody & 2 & 6 & 2 & 2 & 1 & 1 & 2 & 4 & 21.2 & 32.0 \\
\hline 2. Acquaintance & 1 & 4 & 2 & 9 & 6 & 7 & 8 & 4 & 51.5 & 34.0 \\
\hline 3. Close Friend & 2 & 4 & 0 & 2 & 1 & 1 & 4 & 1 & 21.2 & 18.0 \\
\hline 4. Relative & 0 & 0 & 0 & 4 & 2 & 3 & 1 & 1 & 9.1 & 12.0 \\
\hline 5. $\mathrm{Me}$ & 0 & 0 & 0 & 0 & 0 & 0 & 0 & 0 & 0.0 & 0.0 \\
\hline
\end{tabular}


Changing Attitudes 93

\begin{tabular}{|c|c|c|c|c|c|c|c|c|c|c|}
\hline 6. Other & 0 & 1 & 0 & 0 & 0 & 2 & 2 & 0 & 6.1 & 10.0 \\
\hline \multicolumn{11}{|l|}{ Are mentally ill } \\
\hline 1. Nobody & 1 & 4 & 1 & 0 & 3 & 1 & 4 & 1 & 27.3 & 16.0 \\
\hline 2. Acquaintance & 3 & 5 & 2 & 8 & 2 & 5 & 7 & 8 & 42.4 & 54.0 \\
\hline 3. Close Friend & 0 & 4 & 1 & 10 & 0 & 4 & 1 & 0 & 6.1 & 20.0 \\
\hline 4. Relative & 1 & 5 & 1 & 12 & 2 & 2 & 3 & 1 & 21.2 & 24.0 \\
\hline 5. $\mathrm{Me}$ & 0 & 0 & 0 & 8 & 0 & 0 & 0 & 0 & 0.0 & 0.0 \\
\hline 6. Other & 1 & 1 & 0 & 1 & 3 & 3 & 3 & 1 & 21.2 & 10.0 \\
\hline \multicolumn{11}{|l|}{ Are intelligent } \\
\hline 1. Nobody & 0 & 0 & 0 & 11 & 0 & 1 & 0 & 0 & 0.0 & 2.0 \\
\hline 2. Acquaintance & 2 & 8 & 2 & 1 & 5 & 5 & 8 & 6 & 51.5 & 54.0 \\
\hline 3. Close Friend & 3 & 15 & 4 & 2 & 6 & 8 & 11 & 8 & 72.7 & 82.0 \\
\hline 4. Relative & 4 & 11 & 2 & 2 & 6 & 7 & 12 & 7 & 72.7 & 74.0 \\
\hline 5. $\mathrm{Me}$ & 2 & 8 & 2 & 11 & 5 & 5 & 12 & 5 & 63.6 & 52.0 \\
\hline 6. Other & 0 & 1 & 0 & 0 & 2 & 2 & 5 & 3 & 21.2 & 14.0 \\
\hline
\end{tabular}


Appendix 2. Abbreviated Questionnaire Item Acronyms.

\begin{tabular}{|c|c|}
\hline Questionnaire Items: & Abbreviated Questionnaire Items: \\
\hline My overall impression of a person who is obese & Impression: Obese \\
\hline My overall impression of a person who is left handed & Impression: Left handed \\
\hline My overall impression of a person who has a stuttering disorder & Impression: Stuttering \\
\hline My overall impression of a person who is mentally ill & Impression: Mentally ill \\
\hline My overall impression of a person who is intelligent & Impression: Intelligent \\
\hline I want to be a person who is obese & Want: Obese \\
\hline I want to be a person who is left handed & Want: Left handed \\
\hline I want to be a person who has a stuttering disorder & Want: Stuttering \\
\hline I want to be a person who is mentally ill & Want: Mentally ill \\
\hline I want to be a person who is intelligent & Want : Intelligent \\
\hline The amount I know about people who are obese & Knowledge: Obese \\
\hline The amount I know about people who are left handed & Knowledge: Left handed \\
\hline The amount I know about people who have stuttering disorders & Knowledge: Stuttering \\
\hline The amount I know about people who are mentally ill & Knowledge: Mentally ill \\
\hline The amount I know about people who are intelligent & Knowledge: Intelligent \\
\hline People who stutter should try to hide their stuttering & PWS: Should hide their stuttering \\
\hline $\begin{array}{l}\text { People who stutter should have jobs where they have to correctly understand and decide } \\
\text { important things }\end{array}$ & PWS: Should have important jobs \\
\hline People who stutter are nervous or excitable & PWS: Nervous or Excitable \\
\hline People who stutter are shy or fearful & PWS: Shy or fearful \\
\hline People who stutter have themselves to blame for their stuttering & PWS: Should blame self for stuttering \\
\hline
\end{tabular}




\begin{tabular}{|c|c|}
\hline People who stutter can make friends & PWS: Can make friends \\
\hline People who stutter can lead normal lives & PWS: Lead normal lives \\
\hline People who stutter can do any job they want & PWS: Can do any job they want \\
\hline People who stutter are difficult to listen to & PWS: Difficult to listen to \\
\hline If the follow people stuttered, I would be concerned or worried ... my doctor & Concern: Doctor \\
\hline If the follow people stuttered, I would be concerned or worried ... my neighbor & Concern: Neighbor \\
\hline If the follow people stuttered, I would be concerned or worried ... my brother or sister & Concern: Brother or sister \\
\hline If the follow people stuttered, I would be concerned or worried ... my mom or dad & Concern: Mom or dad \\
\hline If the follow people stuttered, I would be concerned or worried ... myself & Concern: Myself \\
\hline $\begin{array}{l}\text { If I were talking to a person who stutters I would try to act like the person was talking } \\
\text { normally }\end{array}$ & Do: Ignore stuttering \\
\hline If I were talking to a person who stutters I would make a joke about stuttering & Do: Joke about stuttering \\
\hline If I were talking to a person who stutters I would fill in the person's words & Do: Fill in words \\
\hline $\begin{array}{l}\text { If I were talking to a person who stutters I would feel impatient (not want to wait while the } \\
\text { person stutters) }\end{array}$ & Do: Feel impatient \\
\hline If I were talking to a person who stutters I would feel comfortable or relaxed & Do: Feel comfortable \\
\hline If I were talking to a person who stutters I would feel pity for the person & Do: Feel pity \\
\hline If I were talking to a person who stutters I would tell the person to "slow down” or "relax" & Do: Say "slow down/Relax" \\
\hline I believe stuttering is caused by genetic inheritance & Cause: Genetics \\
\hline I believe stuttering is caused by ghosts, demons or spirits & Cause: Ghosts, demons, spirits \\
\hline I believe stuttering is caused by being mentally or physically abused & Cause: Mental/Physical Abuse \\
\hline I believe stuttering is caused by trying to think or talk too fast & Cause: Trying to talk, think too fast \\
\hline I believe stuttering is caused by a very frightening event & Cause: Frightening event \\
\hline
\end{tabular}




\begin{tabular}{|c|c|}
\hline I believe stuttering is caused by an act of God & Cause: act of God \\
\hline I believe stuttering is caused by learning or habits & Cause: Learning or habits \\
\hline I believe stuttering is caused by a virus or disease & Cause: Virus or disease \\
\hline I believe stuttering should be helped by other people who stutter & Help: By PWS \\
\hline I believe stuttering should be helped by a speech and language pathologist & Help: By SLP \\
\hline I believe stuttering should be helped by a medical doctor & Help: By doctor \\
\hline My knowledge of stuttering comes from other people who stutter & Info: From PWS \\
\hline My knowledge of stuttering comes from personal experience (me, my family, friends) & Info: Personal Experience \\
\hline My knowledge of stuttering comes from television, radio, films & Info: TV, Radio, Films \\
\hline My knowledge of stuttering comes from magazines, newspapers or books & Info: Magazines, newspapers, books \\
\hline My knowledge of stuttering comes from the internet & Info: Internet \\
\hline My knowledge of stuttering comes from school & Info: School \\
\hline My knowledge of stuttering comes from doctors, nurses or other specialists & Info: Doctors, nurses, specialists \\
\hline In a social situation, I would talk to a person who stutters & Social: Talk to PWS \\
\hline In a social situation, I would be friends with a person who stutters & Social: Friends with PWS \\
\hline In a social situation, I would date a person who stutters & Social: Date PWS \\
\hline In a social situation, I would marry a person who stutters & Social: Marry PWS \\
\hline
\end{tabular}


Appendix 3. Comparisons of PRE versus PRE means, PRE versus PRE mean differences and t test probabilities for PRE-L versus PRE-V, honor classes PRE versus regular classes PRE, males PRE versus females PRE.

\begin{tabular}{|c|c|c|c|c|c|c|c|c|c|c|c|c|}
\hline Sample Size & 40 & 43 & 38 & 45 & 33 & 50 & -- & -- & -- & -- & -- & -- \\
\hline & 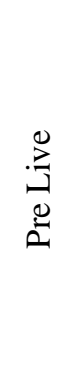 & 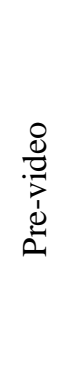 & 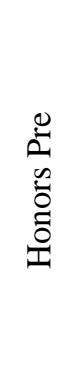 & 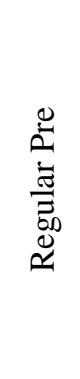 & 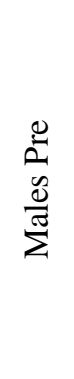 & 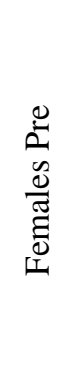 & 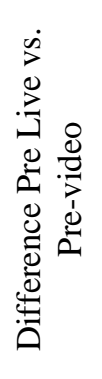 & 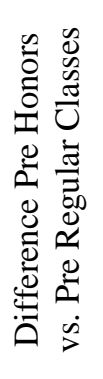 & 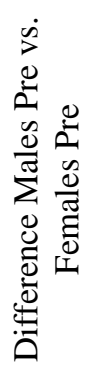 & 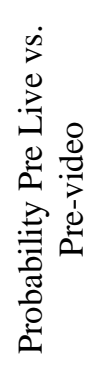 & 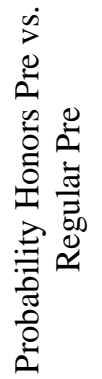 & 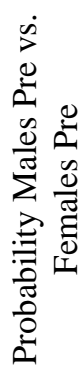 \\
\hline \multicolumn{13}{|l|}{ Demographic Items: } \\
\hline Aspects: Physical health & 68 & 57 & 63 & 61 & 71 & 56 & 11 & 2 & 15 & NS & NS & NS \\
\hline Aspects: Mental health & 73 & 58 & 57 & 72 & 71 & 61 & 14 & 16 & 10 & NS & NS & NS \\
\hline Aspects: Learn new things & 75 & 72 & 71 & 76 & 79 & 70 & 3 & 5 & 9 & NS & NS & NS \\
\hline Aspects: Speaking ability & 70 & 73 & 70 & 73 & 79 & 67 & 3 & 4 & 12 & NS & NS & NS \\
\hline \multicolumn{13}{|l|}{ General Items: } \\
\hline Impression: Obese & -23 & -27 & -18 & -30 & -24 & -25 & 4 & 12 & 1 & NS & NS & NS \\
\hline Impression: Left handed & 24 & 37 & 43 & 21 & 35 & 29 & 13 & 22 & 6 & NS & NS & NS \\
\hline Impression: Stuttering & 6 & 3 & 13 & -2 & 11 & 1 & 3 & 15 & 10 & NS & NS & NS \\
\hline Impression: Mentally ill & -4 & -2 & 3 & -8 & 5 & -8 & 1 & 11 & 13 & NS & NS & NS \\
\hline Impression: Intelligent & 68 & 62 & 63 & 66 & 62 & 66 & 6 & 2 & 4 & NS & NS & NS \\
\hline Want: Obese & -98 & -99 & -96 & -100 & -97 & -99 & 1 & 4 & 2 & NS & NS & NS \\
\hline Want: Left handed & 6 & 7 & 7 & 7 & 18 & -1 & 1 & 0 & 19 & NS & NS & NS \\
\hline Want: Stuttering & -76 & -86 & -76 & -86 & -82 & -81 & 10 & 9 & 1 & NS & NS & NS \\
\hline Want: Mentally ill & -93 & -90 & -87 & -94 & -91 & -91 & 3 & 8 & 0 & NS & NS & NS \\
\hline Want : Intelligent & 78 & 93 & 83 & 88 & 79 & 90 & 16 & 5 & 11 & NS & NS & NS \\
\hline Knowledge: Obese & 13 & 6 & 8 & 10 & 14 & 6 & 7 & 2 & 8 & NS & NS & NS \\
\hline Knowledge: Left handed & -3 & -13 & -5 & -10 & 8 & -18 & 10 & 5 & 27 & NS & NS & NS \\
\hline Knowledge: Stuttering & -41 & -48 & -51 & -38 & -40 & -47 & 6 & 13 & 7 & NS & NS & NS \\
\hline Knowledge: Mentally ill & -18 & -26 & -25 & -19 & -19 & -23 & 8 & 6 & 4 & NS & NS & NS \\
\hline Knowledge: Intelligent & 41 & 43 & 35 & 48 & 53 & 35 & 2 & 13 & 18 & NS & NS & NS \\
\hline \multicolumn{13}{|l|}{ Detailed Items: } \\
\hline PWS: Should hide their stuttering & -84 & -80 & -82 & -49 & -45 & -76 & 4 & 33 & 31 & NS & NS & NS \\
\hline PWS: Should have important jobs & -32 & -31 & 54 & 22 & 38 & 36 & 1 & 32 & 2 & NS & NS & NS \\
\hline PWS: Nervous or Excitable & -36 & -41 & 21 & 24 & 36 & 14 & 4 & 3 & 22 & NS & NS & NS \\
\hline PWS: Shy or fearful & -43 & -40 & 13 & 22 & 12 & 22 & 3 & 9 & 10 & NS & NS & NS \\
\hline
\end{tabular}




\begin{tabular}{|c|c|c|c|c|c|c|c|c|c|c|c|c|}
\hline $\begin{array}{l}\text { PWS: Should blame self for } \\
\text { stuttering }\end{array}$ & -94 & -87 & -87 & -76 & -76 & -84 & 7 & 11 & 8 & NS & NS & NS \\
\hline PWS: Can make friends & -2 & 0 & 95 & 100 & 100 & 96 & 2 & 5 & 4 & NS & NS & NS \\
\hline PWS: Lead normal lives & -2 & -1 & 100 & 93 & 94 & 98 & 1 & 7 & 4 & NS & NS & NS \\
\hline PWS: Can do any job they want & -10 & -13 & 92 & 64 & 70 & 82 & 3 & 28 & 12 & NS & NS & NS \\
\hline PWS: Difficult to listen to & -53 & -53 & 5 & -16 & 0 & -10 & 1 & 21 & 10 & NS & NS & NS \\
\hline Concern: Doctor & -58 & -76 & -37 & -31 & -15 & -46 & 18 & 6 & 31 & NS & NS & NS \\
\hline Concern: Neighbor & -90 & -95 & -84 & -87 & -85 & -86 & 5 & 2 & 1 & NS & NS & NS \\
\hline Concern: Brother or sister & -58 & -73 & -53 & -13 & -33 & -30 & 16 & 39 & 3 & NS & NS & NS \\
\hline Concern: Mom or dad & -58 & -69 & -58 & 0 & -27 & -26 & 11 & 58 & 1 & NS & $*$ & NS \\
\hline Concern: Myself & -38 & -38 & 18 & 29 & 21 & 26 & 1 & 10 & 5 & NS & NS & NS \\
\hline Do: Ignore stuttering & 93 & 91 & 92 & 91 & 91 & 92 & 2 & 1 & 1 & NS & NS & NS \\
\hline Do: Joke about stuttering & -88 & -98 & -89 & -96 & -91 & -94 & 10 & 6 & 3 & NS & NS & NS \\
\hline Do: Fill in words & -30 & -56 & -58 & -31 & -48 & -40 & 26 & 27 & 8 & NS & NS & NS \\
\hline Do: Feel impatient & -63 & -58 & -71 & -51 & -61 & -60 & 4 & 20 & 1 & NS & NS & NS \\
\hline Do: Feel comfortable & 63 & 26 & 61 & 29 & 52 & 38 & 37 & 32 & 14 & NS & NS & NS \\
\hline Do: Feel pity & 13 & -5 & -8 & 13 & -6 & 10 & 17 & 21 & 16 & NS & NS & NS \\
\hline Do: Say "slow down/Relax" & -30 & -26 & -37 & -20 & -18 & -34 & 4 & 17 & 16 & NS & NS & NS \\
\hline Cause: Genetics & 2 & 35 & 24 & 16 & 3 & 30 & 32 & 8 & 27 & NS & NS & NS \\
\hline Cause: Ghosts, demons, spirits & -100 & -91 & -100 & -91 & -91 & -98 & 9 & 9 & 7 & NS & NS & NS \\
\hline Cause: Mental/Physical Abuse & -20 & -23 & -42 & -4 & 0 & -36 & 3 & 38 & 36 & NS & NS & NS \\
\hline $\begin{array}{l}\text { Cause: Trying to talk, think too } \\
\text { fast }\end{array}$ & 33 & 30 & 11 & 49 & 30 & 32 & 2 & 38 & 2 & NS & NS & NS \\
\hline Cause: Frightening event & -18 & 12 & -32 & 22 & 0 & -4 & 29 & 54 & 4 & NS & NS & NS \\
\hline Cause: act of God & -58 & -51 & -45 & -62 & -70 & -44 & 6 & 17 & 26 & NS & NS & NS \\
\hline Cause: Learning or habits & 13 & 26 & -11 & 44 & 24 & 16 & 13 & 55 & 8 & NS & $*$ & NS \\
\hline Cause: Virus or disease & -49 & -58 & -74 & -36 & -53 & -54 & 9 & 37 & 1 & NS & NS & NS \\
\hline Help: By PWS & -2 & -12 & -16 & 0 & -3 & -10 & 9 & 16 & 7 & NS & NS & NS \\
\hline Help: By SLP & 88 & 95 & 84 & 98 & 100 & 86 & 8 & 14 & 14 & NS & NS & NS \\
\hline Help: By doctor & 0 & 16 & 3 & 13 & 0 & 14 & 16 & 11 & 14 & NS & NS & NS \\
\hline Info: From PWS & 23 & -2 & -26 & 41 & 13 & 8 & 25 & 67 & 4 & NS & $*$ & NS \\
\hline Info: Personal Experience & -25 & -37 & -53 & -13 & -18 & -40 & 12 & 39 & 22 & NS & NS & NS \\
\hline Info: TV, Radio, Films & 36 & 12 & 47 & 2 & 13 & 30 & 24 & 45 & 18 & NS & NS & NS \\
\hline $\begin{array}{l}\text { Info: Magazines, newspapers, } \\
\text { books }\end{array}$ & -41 & -28 & -34 & -34 & -34 & -34 & 13 & 0 & 0 & NS & NS & NS \\
\hline Info: Internet & -56 & -63 & -47 & -70 & -53 & -64 & 6 & 23 & 11 & NS & NS & NS \\
\hline Info: School & -8 & -16 & -26 & 0 & 0 & -20 & 9 & 26 & 20 & NS & NS & NS \\
\hline Info: Doctors, nurses, specialists & -72 & -67 & -79 & -61 & -78 & -64 & 4 & 18 & 14 & NS & NS & NS \\
\hline
\end{tabular}




\begin{tabular}{|c|c|c|c|c|c|c|c|c|c|c|c|c|}
\hline Social: Talk to PWS & 93 & 93 & 87 & 98 & 91 & 94 & 1 & 11 & 3 & NS & NS & NS \\
\hline Social: Friends with PWS & 85 & 98 & 97 & 87 & 94 & 90 & 13 & 11 & 4 & NS & NS & NS \\
\hline Social: Date PWS & 45 & 42 & 58 & 31 & 30 & 52 & 3 & 27 & 22 & NS & NS & NS \\
\hline Social: Marry PWS & 45 & 26 & 55 & 18 & 21 & 44 & 19 & 37 & 23 & NS & NS & NS \\
\hline \multicolumn{13}{|l|}{ NS $=$ Not Significant $p>0.05, * p$} \\
\hline Number of significant differences & -- & -- & -- & -- & -- & -- & -- & -- & -- & 0 & 3 & 0 \\
\hline
\end{tabular}


Appendix 4. Comparisons of pre versus post means, pre versus post mean differences and t test probabilities for combined class data PRE-L versus POST-L, PRE-V versus POST-V, POST-V versus POST-V+L, PRE-V versus POST-V+L. The direction of change column is in terms of "positive," "negative,"

"ambiguous" and "no change," "Positive” changes are seen as bold, "negative” changes are seen as not bold, "ambiguous" changes are seen as italics, and "no change” items are seen as underlined.

\begin{tabular}{|c|c|c|c|c|c|c|c|c|c|c|c|c|c|c|c|c|}
\hline \multirow[b]{2}{*}{ Sample Size } & \multicolumn{9}{|c|}{ Difference: Pre vs. Post (All) } & \multicolumn{7}{|c|}{$\begin{array}{l}\text { Difference: Post-V vs. Post-V+L (All), Pre-V vs. Post-V+L } \\
\text { (All) }\end{array}$} \\
\hline & 40 & 40 & 43 & 43 & -- & -- & -- & -- & -- & 43 & 43 & 43 & -- & -- & -- & -- \\
\hline & 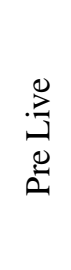 & 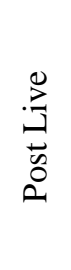 & 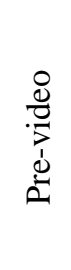 & 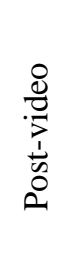 & 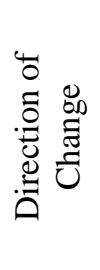 & 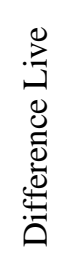 & 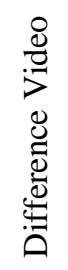 & 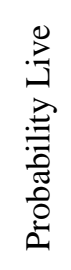 & 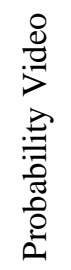 & 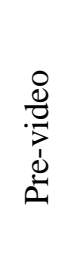 & 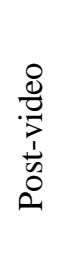 & $\begin{array}{l}+1 \\
\stackrel{+}{>} \\
\stackrel{4}{0} \\
\stackrel{0}{0}\end{array}$ & 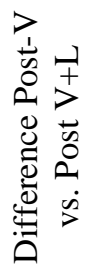 & 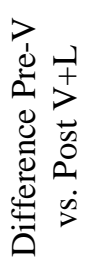 & 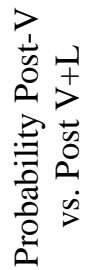 & 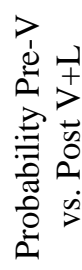 \\
\hline \multicolumn{17}{|l|}{ Demographic Items: } \\
\hline $\begin{array}{l}\text { Aspects: Physical } \\
\text { health }\end{array}$ & 68 & 69 & 57 & 55 & \pm & 1 & 2 & NS & NS & 57 & 55 & 59 & 5 & 2 & NS & NS \\
\hline $\begin{array}{l}\text { Aspects: Mental } \\
\text { health }\end{array}$ & 73 & 68 & 58 & 53 & \pm & 5 & 5 & NS & NS & 58 & 53 & 62 & 8 & 3 & NS & NS \\
\hline $\begin{array}{l}\text { Aspects: Learn new } \\
\text { things }\end{array}$ & 75 & 71 & 72 & 70 & \pm & 4 & 2 & NS & NS & 72 & 70 & 72 & 2 & $\underline{0}$ & NS & $\sim$ \\
\hline $\begin{array}{l}\text { Aspects: Speaking } \\
\text { ability }\end{array}$ & 70 & 70 & 73 & 66 & \pm & 0 & 7 & $\sim$ & NS & 73 & 66 & 65 & 1 & 8 & NS & NS \\
\hline \multicolumn{17}{|l|}{ General Items: } \\
\hline Impression: Obese & -23 & -16 & -27 & -20 & + & 6 & 7 & NS & NS & -27 & -20 & -29 & 9 & 2 & NS & NS \\
\hline $\begin{array}{l}\text { Impression: Left } \\
\text { handed }\end{array}$ & 24 & 19 & 37 & 41 & + & 5 & 3 & NS & NS & 37 & 41 & 25 & 16 & 12 & NS & NS \\
\hline $\begin{array}{l}\text { Impression: } \\
\text { Stuttering }\end{array}$ & 6 & 31 & 3 & 28 & + & 25 & 24 & $*$ & $*$ & 3 & 28 & 10 & 17 & 7 & NS & NS \\
\hline $\begin{array}{l}\text { Impression: Mentally } \\
\text { ill }\end{array}$ & -4 & -3 & -2 & 2 & + & 1 & 5 & NS & NS & -2 & 2 & -5 & 7 & 2 & NS & NS \\
\hline $\begin{array}{l}\text { Impression: } \\
\text { Intelligent }\end{array}$ & 68 & 60 & 62 & 73 & + & 7 & 12 & NS & NS & 62 & 73 & 70 & 3 & 8 & NS & NS \\
\hline Want: Obese & -98 & -93 & -99 & -97 & + & 5 & 2 & NS & NS & -99 & -97 & -94 & 2 & 5 & NS & NS \\
\hline Want: Left handed & 6 & 10 & 7 & 10 & + & 4 & 3 & $\mathrm{NS}$ & NS & 7 & 10 & 7 & 3 & $\underline{0}$ & NS & $\sim$ \\
\hline Want: Stuttering & -76 & -43 & -86 & -56 & + & 34 & 30 & $* *$ & $* * *$ & -86 & -56 & -50 & 6 & 36 & NS & $* * *$ \\
\hline Want: Mentally ill & -93 & -78 & -90 & -84 & + & 14 & 6 & $\mathrm{NS}$ & NS & -90 & -84 & -84 & $\underline{0}$ & 6 & $\sim$ & NS \\
\hline Want : Intelligent & 78 & 71 & 93 & 85 & + & 6 & 8 & NS & NS & 93 & 85 & 77 & 8 & 16 & NS & NS \\
\hline
\end{tabular}




\begin{tabular}{|c|c|c|c|c|c|c|c|c|c|c|c|c|c|c|c|c|}
\hline Knowledge: Obese & 13 & 20 & 6 & 0 & + & 8 & 6 & NS & NS & 6 & 0 & -5 & 5 & 10 & NS & NS \\
\hline $\begin{array}{l}\text { Knowledge: Left } \\
\text { handed }\end{array}$ & -3 & -1 & -13 & -8 & + & 1 & 4 & NS & NS & -13 & -8 & -18 & 10 & 5 & NS & NS \\
\hline $\begin{array}{l}\text { Knowledge: } \\
\text { Stuttering }\end{array}$ & -41 & 23 & -48 & -19 & + & 64 & 29 & $* * *$ & $* *$ & -48 & -19 & -5 & 14 & 43 & NS & $* * *$ \\
\hline $\begin{array}{l}\text { Knowledge: } \\
\text { Mentally ill }\end{array}$ & -18 & -14 & -26 & -20 & + & 3 & 5 & NS & NS & -26 & -20 & -24 & 4 & 1 & NS & NS \\
\hline $\begin{array}{l}\text { Knowledge: } \\
\text { Intelligent }\end{array}$ & 41 & 45 & 43 & 30 & + & 4 & 13 & NS & NS & 43 & 30 & 30 & $\underline{0}$ & 13 & $\sim$ & NS \\
\hline \multicolumn{17}{|l|}{ Detailed Items: } \\
\hline $\begin{array}{l}\text { PWS: Should hide } \\
\text { their stuttering }\end{array}$ & -68 & -83 & -60 & -93 & - & 15 & 33 & NS & $*$ & -60 & -93 & -98 & 5 & 37 & NS & $*$ \\
\hline $\begin{array}{l}\text { PWS: Should have } \\
\text { important jobs }\end{array}$ & 36 & 53 & 37 & 70 & + & 17 & 33 & NS & $*$ & 37 & 70 & 77 & 7 & 40 & NS & $*$ \\
\hline $\begin{array}{l}\text { PWS: Nervous or } \\
\text { Excitable }\end{array}$ & 28 & -70 & 19 & 21 & - & 98 & 2 & $* * *$ & NS & 19 & 21 & -37 & 58 & 56 & $* * *$ & $* *$ \\
\hline PWS: Shy or fearful & 15 & -65 & 21 & 9 & - & 80 & 12 & $* * *$ & NS & 21 & 9 & -28 & 37 & 49 & NS & $*$ \\
\hline $\begin{array}{l}\text { PWS: Should blame } \\
\text { self for stuttering }\end{array}$ & -88 & -100 & -74 & -98 & - & 13 & 23 & NS & NS & -74 & -98 & -95 & 2 & 21 & NS & NS \\
\hline $\begin{array}{l}\text { PWS: Can make } \\
\text { friends }\end{array}$ & 95 & 100 & 100 & 100 & + & 5 & $\underline{0}$ & NS & $\sim$ & 100 & 100 & 100 & $\underline{0}$ & $\underline{0}$ & $\sim$ & $\sim$ \\
\hline $\begin{array}{l}\text { PWS: Lead normal } \\
\text { lives }\end{array}$ & 95 & 100 & 98 & 84 & + & 5 & 14 & NS & NS & 98 & 84 & 98 & 14 & $\underline{0}$ & NS & $\sim$ \\
\hline $\begin{array}{l}\text { PWS: Can do any job } \\
\text { they want }\end{array}$ & 80 & 75 & 74 & 84 & + & 5 & 9 & NS & NS & 74 & 84 & 93 & 9 & 19 & NS & NS \\
\hline $\begin{array}{l}\text { PWS: Difficult to } \\
\text { listen to }\end{array}$ & -5 & -30 & -7 & -51 & - & 25 & 44 & NS & $*$ & -7 & -51 & -72 & 21 & 65 & NS & $* * *$ \\
\hline Concern: Doctor & -15 & -35 & -51 & -67 & - & 20 & 16 & NS & NS & -51 & -67 & -67 & $\underline{0}$ & 16 & $\sim$ & NS \\
\hline Concern: Neighbor & -80 & -90 & -91 & -93 & - & 10 & 2 & NS & NS & -91 & -93 & -95 & 2 & 5 & NS & NS \\
\hline $\begin{array}{l}\text { Concern: Brother or } \\
\text { sister }\end{array}$ & -15 & -48 & -47 & -63 & - & 33 & 16 & NS & NS & -47 & -63 & -63 & $\underline{0}$ & 16 & $\sim$ & NS \\
\hline $\begin{array}{l}\text { Concern: Mom or } \\
\text { dad }\end{array}$ & -15 & -53 & -37 & -65 & - & 38 & 28 & $*$ & NS & -37 & -65 & -63 & 2 & 26 & NS & NS \\
\hline Concern: Myself & 25 & -28 & 23 & -7 & - & 53 & 30 & $*$ & NS & 23 & -7 & 12 & 19 & 12 & NS & NS \\
\hline Do: Ignore stuttering & 93 & 83 & 91 & 84 & + & 10 & 7 & NS & NS & 91 & 84 & 88 & 5 & 2 & NS & NS \\
\hline $\begin{array}{l}\text { Do: Joke about } \\
\text { stuttering }\end{array}$ & -88 & -93 & -98 & -95 & - & 5 & 2 & NS & NS & -98 & -95 & -93 & 2 & 5 & NS & NS \\
\hline Do: Fill in words & -30 & -85 & -56 & -51 & - & 55 & 5 & $* * *$ & NS & -56 & -51 & -63 & 12 & 7 & NS & $\mathrm{NS}$ \\
\hline Do: Feel impatient & -63 & -90 & -58 & -77 & - & 28 & 19 & $\mathrm{NS}$ & NS & -58 & -77 & -81 & 5 & 23 & NS & $\mathrm{NS}$ \\
\hline
\end{tabular}




\begin{tabular}{|c|c|c|c|c|c|c|c|c|c|c|c|c|c|c|c|c|}
\hline Do: Feel comfortable & 63 & 63 & 26 & 69 & + & $\underline{0}$ & 43 & $\sim$ & $*$ & 26 & 69 & 67 & 2 & 42 & NS & $*$ \\
\hline Do: Feel pity & 13 & -25 & -5 & -16 & - & 38 & 12 & $\mathrm{NS}$ & NS & -5 & -16 & -37 & 21 & 33 & NS & NS \\
\hline $\begin{array}{l}\text { Do: Say "slow } \\
\text { down/Relax" }\end{array}$ & -30 & -78 & -26 & -44 & - & 48 & 19 & $*$ & NS & -26 & -44 & -62 & 18 & 36 & NS & $*$ \\
\hline Cause: Genetics & 2 & 78 & 35 & -12 & + & 75 & 47 & $* * *$ & $*$ & 35 & -12 & 74 & 86 & 40 & $* * *$ & NS \\
\hline $\begin{array}{l}\text { Cause: Ghosts, } \\
\text { demons, spirits }\end{array}$ & -100 & -100 & -91 & -91 & - & $\underline{0}$ & $\underline{0}$ & $\sim$ & $\sim$ & -91 & -91 & -95 & 5 & 5 & NS & NS \\
\hline $\begin{array}{l}\text { Cause: } \\
\text { Mental/Physical } \\
\text { Abuse }\end{array}$ & -20 & -63 & -23 & -44 & - & 43 & 21 & $*$ & NS & -23 & -44 & -63 & 19 & 40 & NS & $*$ \\
\hline $\begin{array}{l}\text { Cause: Trying to } \\
\text { talk, think too fast }\end{array}$ & 33 & -55 & 30 & -7 & - & 88 & 37 & $* * *$ & NS & 30 & -7 & -49 & 42 & 79 & $*$ & $* * *$ \\
\hline $\begin{array}{l}\text { Cause: Frightening } \\
\text { event }\end{array}$ & -18 & -65 & 12 & -40 & - & 48 & 51 & $*$ & $* *$ & 12 & -40 & -70 & 30 & 81 & NS & $* * *$ \\
\hline Cause: act of God & -58 & -60 & -51 & -63 & - & 3 & 12 & $\mathrm{NS}$ & NS & -51 & -63 & -74 & 12 & 23 & NS & NS \\
\hline $\begin{array}{l}\text { Cause: Learning or } \\
\text { habits }\end{array}$ & 13 & -78 & 26 & -23 & - & 90 & 49 & $* * *$ & $* *$ & 26 & -23 & -65 & 42 & 91 & $*$ & $* * *$ \\
\hline $\begin{array}{l}\text { Cause: Virus or } \\
\text { disease }\end{array}$ & -49 & -97 & -58 & -79 & - & 49 & 21 & $* *$ & NS & -58 & -79 & -95 & 16 & 37 & NS & $* *$ \\
\hline Help: By PWS & -2 & 28 & -12 & 74 & + & 30 & 86 & NS & $* * *$ & -12 & 74 & 77 & 2 & 88 & NS & $* * *$ \\
\hline Help: By SLP & 88 & 83 & 95 & 93 & + & 5 & 2 & NS & NS & 95 & 93 & 93 & $\underline{0}$ & 2 & $\sim$ & NS \\
\hline Help: By doctor & 0 & -23 & 16 & -5 & - & 23 & 21 & $\mathrm{NS}$ & NS & 16 & -5 & -19 & 14 & 35 & NS & NS \\
\hline Info: From PWS & 23 & 80 & -2 & -21 & \pm & 57 & 19 & $\mathrm{NS}$ & NS & -2 & -21 & 47 & 67 & 49 & $* *$ & $*$ \\
\hline $\begin{array}{l}\text { Info: Personal } \\
\text { Experience }\end{array}$ & -25 & -23 & -37 & -49 & \pm & 2 & 12 & NS & NS & -37 & -49 & -58 & 9 & 21 & NS & NS \\
\hline $\begin{array}{l}\text { Info: TV, Radio, } \\
\text { Films }\end{array}$ & 36 & -5 & 12 & 77 & \pm & 41 & 65 & NS & $* * *$ & 12 & 77 & 49 & 28 & 37 & NS & NS \\
\hline $\begin{array}{l}\text { Info: Magazines, } \\
\text { newspapers, books }\end{array}$ & -41 & -30 & -28 & -37 & \pm & 11 & 9 & NS & NS & -28 & -37 & -42 & 5 & 14 & NS & NS \\
\hline Info: Internet & -56 & -60 & -63 & -40 & \pm & 4 & 23 & NS & NS & -63 & -40 & -52 & 13 & 10 & $\mathrm{NS}$ & NS \\
\hline Info: School & -8 & 35 & -16 & 30 & \pm & 43 & 47 & NS & NS & -16 & 30 & 44 & 14 & 60 & NS & $*$ \\
\hline $\begin{array}{l}\text { Info: Doctors, nurses, } \\
\text { specialists }\end{array}$ & -72 & -68 & -67 & -74 & \pm & 4 & 7 & NS & NS & -67 & -74 & -81 & 7 & 14 & NS & NS \\
\hline Social: Talk to PWS & 93 & 95 & 93 & 100 & + & 3 & 7 & NS & NS & 93 & 100 & 100 & $\underline{0}$ & 7 & NS & NS \\
\hline $\begin{array}{l}\text { Social: Friends with } \\
\text { PWS }\end{array}$ & 85 & 95 & 98 & 100 & + & 10 & 2 & NS & NS & 98 & 100 & 100 & $\underline{0}$ & 2 & $\sim$ & NS \\
\hline Social: Date PWS & 45 & 55 & 42 & 63 & + & 10 & 21 & NS & NS & 42 & 63 & 60 & 2 & 19 & NS & NS \\
\hline Social: Marry PWS & 45 & 48 & 26 & 49 & + & 2 & 23 & NS & NS & 26 & 49 & 51 & 2 & 26 & NS & NS \\
\hline
\end{tabular}


Changing Attitudes 103

\begin{tabular}{|c|c|c|c|c|c|c|c|c|c|c|c|c|c|c|c|c|}
\hline \multicolumn{17}{|c|}{ SUMMARY } \\
\hline $\begin{array}{l}\text { Number of "Positive } \\
\text { Change" }\end{array}$ & -- & -- & -- & -- & -- & 43 & 37 & -- & -- & -- & -- & -- & 31 & 38 & -- & -- \\
\hline $\begin{array}{l}\text { Number of "Negative } \\
\text { Change" }\end{array}$ & -- & -- & -- & -- & -- & 6 & 12 & -- & -- & -- & -- & -- & 16 & 13 & -- & -- \\
\hline $\begin{array}{l}\text { Number of "No" } \\
\text { Change }\end{array}$ & -- & -- & -- & -- & -- & 2 & 2 & -- & -- & -- & -- & -- & 8 & 4 & -- & -- \\
\hline $\begin{array}{l}\text { Number of } \\
\text { “Ambiguous” } \\
\text { Change }\end{array}$ & -- & -- & -- & -- & -- & 11 & 11 & -- & -- & -- & -- & -- & 7 & 7 & -- & -- \\
\hline $\begin{array}{l}\text { Number of } \\
\text { significant } \\
\text { differences }\end{array}$ & -- & -- & -- & -- & -- & -- & -- & 15 & 12 & -- & -- & -- & -- & -- & 5 & 17 \\
\hline
\end{tabular}


Appendix 5. Comparisons of pre versus post means, pre versus post mean differences and t test probabilities for male and female data PRE-L versus POST-L. The direction of change column is in terms of "positive," "negative," "ambiguous" and "no change," "Positive” changes are seen as bold, "negative” changes are seen as not bold, "ambiguous" changes are seen as italics, and "no change" items are seen as underlined.

\begin{tabular}{|c|c|c|c|c|c|c|c|c|c|}
\hline \multirow[b]{2}{*}{ Column Number } & \multicolumn{2}{|c|}{ Pre Live } & \multicolumn{2}{|c|}{ Post Live } & \multirow{2}{*}{$\begin{array}{c}-- \\
5 \\
\end{array}$} & \multirow{2}{*}{$\begin{array}{l}-- \\
6\end{array}$} & \multirow{2}{*}{$\begin{array}{l}-- \\
7\end{array}$} & \multirow{2}{*}{$\begin{array}{l}-- \\
8\end{array}$} & \multirow{2}{*}{$\begin{array}{c}-- \\
9\end{array}$} \\
\hline & 1 & 2 & 3 & 4 & & & & & \\
\hline Sample Size & 14 & 26 & 14 & 26 & -- & -- & -- & -- & -- \\
\hline \multicolumn{10}{|l|}{ Demographic Items: } \\
\hline Aspects: Physical health & 75 & 63 & 71 & 67 & \pm & 4 & 4 & NS & NS \\
\hline Aspects: Mental health & 75 & 71 & 82 & 60 & \pm & 7 & 12 & NS & NS \\
\hline Aspects: Learn new things & 82 & 71 & 82 & 65 & \pm & 0 & 6 & NS & NS \\
\hline Impression: Stuttering & 0 & 10 & 36 & 29 & + & 36 & 19 & NS & NS \\
\hline Impression: Mentally ill & -11 & 0 & 4 & -6 & + & 14 & 6 & NS & NS \\
\hline Impression: Intelligent & 57 & 73 & 46 & 67 & + & 11 & 6 & NS & NS \\
\hline Want: Obese & -96 & -98 & -86 & -96 & + & 11 & 2 & NS & NS \\
\hline Want: Left handed & 21 & -2 & 14 & 8 & + & 7 & 10 & NS & NS \\
\hline Want: Stuttering & -75 & -77 & -32 & -48 & + & 43 & 29 & NS & $*$ \\
\hline Want: Mentally ill & -89 & -94 & -62 & -87 & + & 28 & 8 & NS & NS \\
\hline Want : Intelligent & 61 & 87 & 61 & 77 & + & $\underline{0}$ & 10 & NS & NS \\
\hline Knowledge: Obese & 14 & 12 & 25 & 17 & + & 11 & 6 & NS & NS \\
\hline Knowledge: Left handed & 8 & -8 & 7 & -6 & + & 1 & 2 & NS & NS \\
\hline Knowledge: Stuttering & -36 & -44 & 21 & 23 & + & 57 & 67 & NS & $* * *$ \\
\hline
\end{tabular}




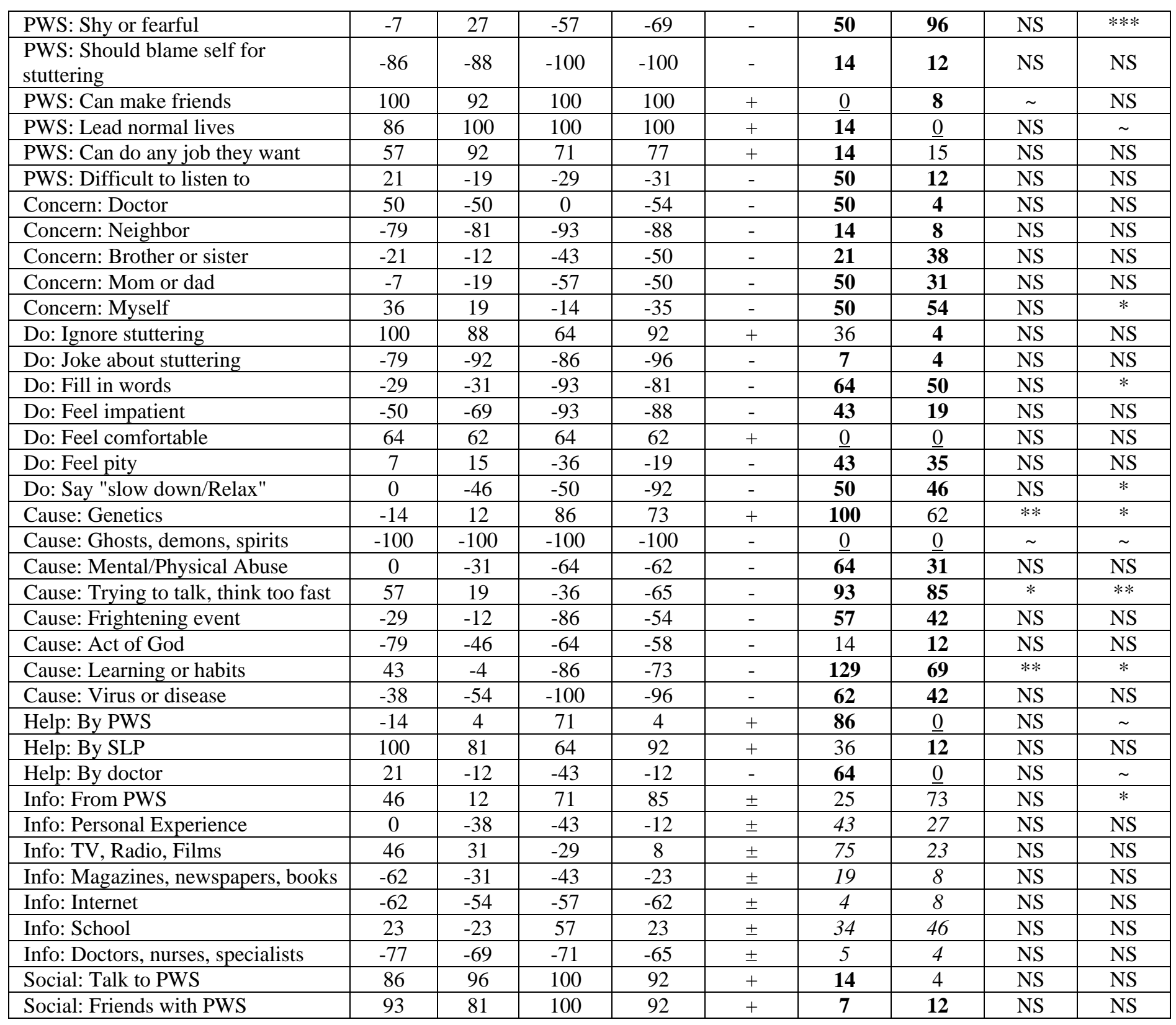




\begin{tabular}{|c|c|c|c|c|c|c|c|c|c|}
\hline Social: Date PWS & 7 & 65 & 14 & 77 & + & 7 & 12 & NS & NS \\
\hline Social: Marry PWS & 7 & 65 & 14 & 65 & + & 7 & $\underline{0}$ & NS & $\sim$ \\
\hline \multicolumn{10}{|c|}{ SUMMARY } \\
\hline Number of "Positive Change" & -- & -- & -- & -- & -- & 40 & 35 & -- & -- \\
\hline Number of "Negative Change" & -- & -- & -- & -- & -- & 7 & 10 & -- & -- \\
\hline Number of "No" Change & -- & -- & -- & -- & -- & 4 & 6 & -- & -- \\
\hline Number of “Ambiguous” Change & -- & -- & -- & -- & -- & 11 & 11 & -- & -- \\
\hline Number of significant differences & -- & -- & -- & -- & -- & -- & -- & 4 & 11 \\
\hline
\end{tabular}


Appendix 6. Comparisons of pre versus post means, pre versus post mean differences and t test probabilities for male and female PRE-V versus POST-V, POSTV versus POST-V+L, PRE-V versus POST-V+L. The direction of change column is in terms of "positive," "negative," "ambiguous” and "no change,"

"Positive" changes are seen as bold, "negative" changes are seen as not bold, "ambiguous" changes are seen as italics, and "no change" items are seen as underlined.

\begin{tabular}{|c|c|c|c|c|c|c|c|c|c|c|c|c|c|c|c|c|c|c|c|}
\hline \multirow{2}{*}{\begin{tabular}{|l|} 
\\
Column \\
Number \\
\end{tabular}} & \multicolumn{2}{|c|}{ Pre Video } & \multicolumn{2}{|c|}{ Post Video } & \multicolumn{2}{|c|}{$\begin{array}{c}\text { Post V + } \\
\text { Live } \\
\end{array}$} & \multirow{2}{*}{--} & \multirow{2}{*}{--} & \multirow{2}{*}{--} & \multirow{2}{*}{--} & \multirow{2}{*}{--} & \multirow{2}{*}{--} & \multirow{2}{*}{$\begin{array}{c}-- \\
13\end{array}$} & \multirow{2}{*}{--} & \multirow{2}{*}{--} & \multirow{2}{*}{--} & \multirow{2}{*}{--} & \multirow{2}{*}{--} & \multirow{2}{*}{$\begin{array}{l}-- \\
19\end{array}$} \\
\hline & 1 & 2 & 3 & 4 & 5 & 6 & & & & & & & & & & & & & \\
\hline \multirow{2}{*}{ Sample Size } & 19 & 24 & 19 & 24 & 19 & 24 & -- & -- & -- & -- & -- & -- & -- & -- & -- & -- & -- & -- & -- \\
\hline & $\frac{0}{\pi}$ & 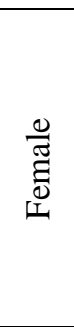 & $\frac{0}{\sum^{\pi}}$ & 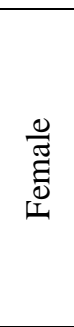 & $\frac{0}{\pi}$ & $\underset{\widetilde{\Xi}}{\widetilde{\Xi}}$ & 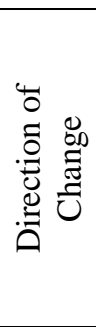 & 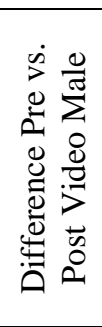 & 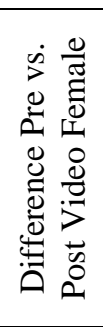 & 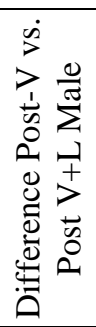 & 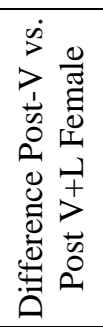 & 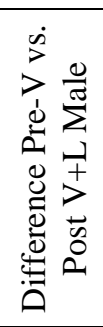 & 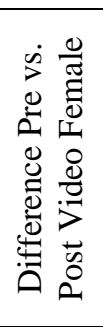 & 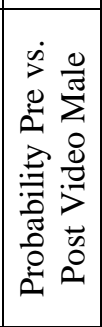 & 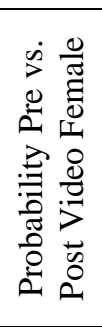 & 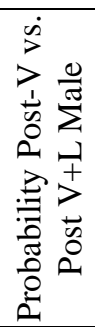 & 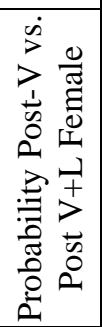 & 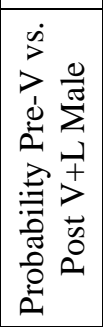 & 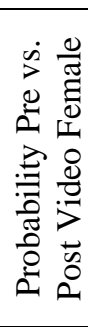 \\
\hline \multicolumn{20}{|l|}{$\begin{array}{l}\text { Demographic } \\
\text { Items: }\end{array}$} \\
\hline $\begin{array}{l}\text { Aspects: } \\
\text { Physical health }\end{array}$ & 68 & 48 & 74 & 40 & 76 & 46 & \pm & 5 & 8 & 3 & 6 & 8 & 2 & NS & NS & NS & NS & NS & NS \\
\hline $\begin{array}{l}\text { Aspects: } \\
\text { Mental health }\end{array}$ & 68 & 50 & 66 & 44 & 79 & 48 & \pm & 3 & 6 & 13 & 4 & 11 & 2 & NS & NS & NS & NS & NS & NS \\
\hline $\begin{array}{l}\text { Aspects: Learn } \\
\text { new things }\end{array}$ & 76 & 69 & 71 & 69 & 76 & 69 & \pm & 5 & $\underline{0}$ & 5 & $\underline{0}$ & $\underline{0}$ & $\underline{0}$ & NS & NS & NS & NS & NS & NS \\
\hline $\begin{array}{l}\text { Aspects: } \\
\text { Speaking } \\
\text { ability }\end{array}$ & 84 & 65 & 71 & 63 & 74 & 58 & \pm & 13 & 2 & 3 & 4 & 11 & 6 & NS & NS & NS & NS & NS & NS \\
\hline \multicolumn{20}{|l|}{ General Items: } \\
\hline $\begin{array}{l}\text { Impression: } \\
\text { Obese }\end{array}$ & -24 & -29 & -11 & -27 & -24 & -33 & + & 13 & 2 & 13 & 6 & $\underline{0}$ & 4 & NS & NS & NS & NS & NS & NS \\
\hline $\begin{array}{l}\text { Impression: } \\
\text { Left handed }\end{array}$ & 45 & 31 & 50 & 33 & 25 & 25 & + & 5 & 2 & 25 & 8 & 20 & 6 & NS & NS & NS & NS & NS & NS \\
\hline $\begin{array}{l}\text { Impression: } \\
\text { Stuttering }\end{array}$ & 18 & -8 & 34 & 23 & 16 & 6 & + & 16 & 31 & 18 & 17 & 3 & 15 & NS & $*$ & NS & NS & NS & NS \\
\hline $\begin{array}{l}\text { Impression: } \\
\text { Mentally ill }\end{array}$ & 17 & -17 & 11 & -4 & 6 & -13 & + & 6 & 13 & 5 & 8 & 11 & 4 & NS & NS & NS & NS & NS & NS \\
\hline $\begin{array}{l}\text { Impression: } \\
\text { Intelligent }\end{array}$ & 66 & 58 & 74 & 73 & 68 & 71 & + & 8 & 15 & 5 & 2 & 3 & 13 & NS & NS & NS & NS & NS & NS \\
\hline
\end{tabular}


Changing Attitudes 108

\begin{tabular}{|c|c|c|c|c|c|c|c|c|c|c|c|c|c|c|c|c|c|c|c|}
\hline Want: Obese & -97 & -100 & -95 & -98 & -89 & -98 & + & 3 & 2 & 5 & $\underline{0}$ & 8 & 2 & NS & NS & NS & NS & NS & $\mathrm{NS}$ \\
\hline $\begin{array}{l}\text { Want: Left } \\
\text { handed }\end{array}$ & 16 & 0 & 18 & 4 & 13 & 2 & + & 3 & 4 & 5 & 2 & 3 & 2 & NS & NS & NS & NS & NS & NS \\
\hline $\begin{array}{l}\text { Want: } \\
\text { Stuttering }\end{array}$ & -87 & -85 & -66 & -48 & -58 & -44 & + & 21 & 38 & 8 & 4 & 29 & 42 & NS & $* *$ & NS & NS & $*$ & $* *$ \\
\hline $\begin{array}{l}\text { Want: Mentally } \\
\text { ill }\end{array}$ & -92 & -88 & -92 & -77 & -87 & -81 & + & $\underline{0}$ & 10 & 5 & 4 & 5 & 6 & NS & NS & NS & NS & NS & NS \\
\hline $\begin{array}{l}\text { Want : } \\
\text { Intelligent }\end{array}$ & 92 & 94 & 89 & 81 & 82 & 73 & + & 3 & 13 & 8 & 8 & 11 & 21 & NS & NS & NS & NS & NS & NS \\
\hline $\begin{array}{l}\text { Knowledge: } \\
\text { Obese }\end{array}$ & 13 & 0 & 8 & -6 & 0 & -8 & + & 5 & 6 & 8 & 2 & 13 & 8 & NS & NS & NS & NS & NS & NS \\
\hline $\begin{array}{l}\text { Knowledge: } \\
\text { Left handed }\end{array}$ & 8 & -30 & 11 & -23 & -8 & -26 & + & 2 & 7 & 18 & 3 & 16 & 3 & NS & NS & NS & NS & NS & NS \\
\hline $\begin{array}{l}\text { Knowledge: } \\
\text { Stuttering }\end{array}$ & -44 & -50 & -18 & -19 & 0 & -8 & + & 26 & 31 & 18 & 10 & 44 & 42 & NS & $*$ & NS & NS & NS & $*$ \\
\hline $\begin{array}{l}\text { Knowledge: } \\
\text { Mentally ill }\end{array}$ & -24 & -27 & -16 & -24 & -29 & -21 & + & 8 & 3 & 13 & 3 & 5 & 6 & NS & NS & NS & NS & NS & NS \\
\hline $\begin{array}{l}\text { Knowledge: } \\
\text { Intelligent }\end{array}$ & 61 & 29 & 37 & 25 & 29 & 31 & + & 24 & 4 & 8 & 6 & 32 & 2 & NS & NS & NS & NS & NS & NS \\
\hline \multicolumn{20}{|l|}{$\begin{array}{l}\text { Detailed } \\
\text { Items: }\end{array}$} \\
\hline $\begin{array}{l}\text { PWS: Should } \\
\text { hide their } \\
\text { stuttering }\end{array}$ & -42 & -75 & -89 & -96 & -100 & -96 & - & 47 & 21 & 11 & $\underline{0}$ & 58 & 21 & NS & NS & NS & $\sim$ & NS & NS \\
\hline $\begin{array}{l}\text { PWS: Should } \\
\text { have important } \\
\text { jobs }\end{array}$ & 32 & 42 & 68 & 71 & 68 & 83 & + & 37 & 29 & $\underline{0}$ & 13 & 37 & 42 & NS & NS & NS & NS & NS & NS \\
\hline $\begin{array}{l}\text { PWS: Nervous } \\
\text { or Excitable }\end{array}$ & 32 & 8 & 53 & -4 & -26 & -46 & - & 21 & 13 & 79 & 42 & 58 & 54 & NS & NS & * & $*$ & $*$ & NS \\
\hline $\begin{array}{l}\text { PWS: Shy or } \\
\text { fearful }\end{array}$ & 26 & 17 & 21 & 0 & -16 & -38 & - & 5 & 17 & 37 & 38 & 42 & 54 & NS & NS & NS & NS & NS & $*$ \\
\hline $\begin{array}{l}\text { PWS: Should } \\
\text { blame self for } \\
\text { stuttering }\end{array}$ & -68 & -79 & -95 & -100 & -100 & -92 & - & 26 & 21 & 5 & 8 & 32 & 13 & NS & NS & NS & NS & NS & NS \\
\hline $\begin{array}{l}\text { PWS: Can } \\
\text { make friends }\end{array}$ & 100 & 100 & 100 & 100 & 100 & 100 & + & $\underline{0}$ & $\underline{0}$ & $\underline{0}$ & $\underline{0}$ & $\underline{0}$ & $\underline{0}$ & $\sim$ & $\sim$ & $\sim$ & $\sim$ & $\sim$ & $\sim$ \\
\hline $\begin{array}{l}\text { PWS: Lead } \\
\text { normal lives }\end{array}$ & 100 & 96 & 79 & 88 & 100 & 96 & + & 21 & 8 & 21 & 8 & $\underline{0}$ & $\underline{0}$ & NS & NS & NS & NS & $\sim$ & $\sim$ \\
\hline PWS: Can do & 79 & 71 & 100 & 71 & 100 & 88 & + & 21 & $\underline{0}$ & $\underline{0}$ & 17 & 21 & 17 & NS & $\mathrm{NS}$ & $\sim$ & $\mathrm{NS}$ & NS & $\mathrm{NS}$ \\
\hline
\end{tabular}




\begin{tabular}{|c|c|c|c|c|c|c|c|c|c|c|c|c|c|c|c|c|c|c|c|}
\hline $\begin{array}{l}\text { any job they } \\
\text { want }\end{array}$ & & & & & & & & & & & & & & & & & & & \\
\hline $\begin{array}{l}\text { PWS: Difficult } \\
\text { to listen to }\end{array}$ & -16 & 0 & -21 & -75 & -68 & -75 & - & 5 & 75 & 47 & $\underline{0}$ & 53 & 75 & NS & $* *$ & NS & NS & NS & $* *$ \\
\hline $\begin{array}{l}\text { Concern: } \\
\text { Doctor }\end{array}$ & -63 & -42 & -63 & -71 & -74 & -63 & - & $\underline{0}$ & 29 & 11 & 8 & 11 & 21 & NS & NS & NS & NS & NS & NS \\
\hline $\begin{array}{l}\text { Concern: } \\
\text { Neighbor }\end{array}$ & -89 & -92 & -95 & -92 & -95 & -96 & - & 5 & $\underline{0}$ & $\underline{0}$ & 4 & 5 & 4 & NS & NS & NS & NS & NS & NS \\
\hline $\begin{array}{l}\text { Concern: } \\
\text { Brother or sister }\end{array}$ & -42 & -50 & -63 & -63 & -79 & -50 & - & 21 & 13 & 16 & 13 & 37 & $\underline{0}$ & NS & NS & NS & NS & NS & NS \\
\hline $\begin{array}{l}\text { Concern: Mom } \\
\text { or dad }\end{array}$ & -42 & -33 & -63 & -67 & -79 & -50 & - & 21 & 33 & 16 & 17 & 37 & 17 & NS & NS & NS & NS & NS & NS \\
\hline $\begin{array}{l}\text { Concern: } \\
\text { Myself }\end{array}$ & 11 & 33 & -21 & 4 & 16 & 8 & - & 32 & 29 & 37 & 4 & 5 & 25 & NS & NS & NS & NS & NS & NS \\
\hline $\begin{array}{l}\text { Do: Ignore } \\
\text { stuttering }\end{array}$ & 84 & 96 & 84 & 83 & 79 & 96 & + & $\underline{0}$ & 13 & 5 & 13 & 5 & $\underline{0}$ & NS & NS & NS & NS & NS & NS \\
\hline $\begin{array}{l}\text { Do: Joke about } \\
\text { stuttering }\end{array}$ & -100 & -96 & -100 & -92 & -100 & -88 & - & $\underline{0}$ & 4 & $\underline{0}$ & 4 & $\underline{0}$ & 8 & $\sim$ & NS & $\sim$ & NS & $\sim$ & NS \\
\hline $\begin{array}{l}\text { Do: Fill in } \\
\text { words }\end{array}$ & -63 & -50 & -37 & -63 & -58 & -67 & - & 26 & 13 & 21 & 4 & 5 & 17 & NS & NS & NS & NS & NS & NS \\
\hline $\begin{array}{l}\text { Do: Feel } \\
\text { impatient }\end{array}$ & -68 & -50 & -84 & -71 & -84 & -79 & - & 16 & 21 & $\underline{0}$ & 8 & 16 & 29 & NS & NS & NS & NS & NS & NS \\
\hline $\begin{array}{l}\text { Do: Feel } \\
\text { comfortable }\end{array}$ & 42 & 13 & 84 & 57 & 79 & 58 & + & 42 & 44 & 5 & 2 & 37 & 46 & NS & NS & NS & NS & NS & NS \\
\hline Do: Feel pity & -16 & 4 & -11 & -21 & -21 & -50 & - & 5 & 25 & 11 & 29 & 5 & 54 & NS & NS & NS & NS & NS & $*$ \\
\hline $\begin{array}{l}\text { Do: Say "slow } \\
\text { down/Relax" }\end{array}$ & -32 & -21 & -32 & -54 & -56 & -67 & - & $\underline{0}$ & 33 & 24 & 13 & 24 & 46 & NS & NS & NS & NS & NS & $*$ \\
\hline $\begin{array}{l}\text { Cause: } \\
\text { Genetics }\end{array}$ & 16 & 50 & -21 & -4 & 84 & 67 & + & 37 & 54 & 105 & 71 & 68 & 17 & NS & NS & $* *$ & $*$ & $*$ & NS \\
\hline $\begin{array}{l}\text { Cause: Ghosts, } \\
\text { demons, spirits }\end{array}$ & -84 & -96 & -79 & -100 & -89 & -100 & - & 5 & 4 & 11 & $\underline{0}$ & 5 & 4 & NS & NS & NS & $\sim$ & NS & NS \\
\hline $\begin{array}{l}\text { Cause: } \\
\text { Mental/Physical } \\
\text { Abuse } \\
\end{array}$ & 0 & -42 & -42 & -46 & -79 & -50 & - & 42 & 4 & 37 & 4 & 79 & 8 & NS & NS & NS & NS & $*$ & NS \\
\hline $\begin{array}{l}\text { Cause: Trying } \\
\text { to talk, think } \\
\text { too fast }\end{array}$ & 11 & 46 & 11 & -21 & -47 & -50 & - & $\underline{0}$ & 67 & 58 & 29 & 58 & 96 & NS & $*$ & NS & NS & NS & $* * *$ \\
\hline $\begin{array}{l}\text { Cause: } \\
\text { Frightening }\end{array}$ & 21 & 4 & -37 & -42 & -79 & -63 & - & 58 & 46 & 42 & 21 & 100 & 67 & NS & NS & NS & NS & $* *$ & $*$ \\
\hline
\end{tabular}


Changing Attitudes 110

\begin{tabular}{|c|c|c|c|c|c|c|c|c|c|c|c|c|c|c|c|c|c|c|c|}
\hline event & & & & & & & & & & & & & & & & & & & \\
\hline $\begin{array}{l}\text { Cause: act of } \\
\text { God }\end{array}$ & -63 & -42 & -68 & -58 & -79 & -71 & - & 5 & 17 & 11 & 13 & 16 & 29 & NS & NS & NS & NS & NS & NS \\
\hline $\begin{array}{l}\text { Cause: } \\
\text { Learning or } \\
\text { habits }\end{array}$ & 11 & 38 & -21 & -25 & -74 & -58 & - & 32 & 63 & 53 & 33 & 84 & 96 & NS & $*$ & $*$ & NS & $*$ & $* * *$ \\
\hline $\begin{array}{l}\text { Cause: Virus or } \\
\text { disease }\end{array}$ & -63 & -54 & -74 & -83 & -95 & -96 & - & 11 & 29 & 21 & 13 & 32 & 42 & NS & NS & NS & NS & NS & $*$ \\
\hline Help: By PWS & 5 & -25 & 68 & 79 & 68 & 83 & + & 63 & 104 & $\underline{0}$ & 4 & 63 & 108 & NS & $* * *$ & NS & NS & NS & $* * *$ \\
\hline Help: By SLP & 100 & 92 & 95 & 92 & 95 & 92 & + & 5 & $\underline{0}$ & $\underline{0}$ & $\underline{0}$ & 5 & $\underline{0}$ & NS & NS & $\sim$ & NS & NS & NS \\
\hline Help: By doctor & -16 & 42 & -32 & 17 & -37 & -4 & - & 16 & 25 & 5 & 21 & 21 & $\overline{46}$ & NS & NS & NS & NS & NS & NS \\
\hline $\begin{array}{l}\text { Info: From } \\
\text { PWS }\end{array}$ & -11 & 4 & -11 & -29 & 58 & 38 & \pm & 0 & 33 & 68 & 67 & 68 & 33 & NS & NS & NS & NS & NS & NS \\
\hline $\begin{array}{l}\text { Info: Personal } \\
\text { Experience }\end{array}$ & -32 & -42 & -47 & -50 & -58 & -58 & \pm & 16 & 8 & 11 & 8 & 26 & 17 & NS & NS & NS & NS & NS & NS \\
\hline $\begin{array}{l}\text { Info: TV, } \\
\text { Radio, Films }\end{array}$ & -11 & 29 & 68 & 83 & 26 & 67 & \pm & 79 & 54 & 42 & 17 & 37 & 38 & $*$ & NS & NS & NS & NS & NS \\
\hline $\begin{array}{l}\text { Info: } \\
\text { Magazines, } \\
\text { newspapers, } \\
\text { books }\end{array}$ & -16 & -38 & -26 & -46 & -47 & -38 & \pm & 11 & 8 & 21 & 8 & 32 & 0 & NS & NS & NS & NS & NS & NS \\
\hline Info: Internet & -47 & -75 & -37 & -42 & -47 & -57 & \pm & 11 & 33 & 11 & 15 & 0 & 18 & NS & NS & NS & NS & NS & NS \\
\hline Info: School & -16 & -17 & 47 & 17 & 89 & 8 & \pm & 63 & 33 & 42 & 8 & 105 & 25 & NS & NS & NS & NS & $* *$ & NS \\
\hline $\begin{array}{l}\text { Info: Doctors, } \\
\text { nurses, } \\
\text { specialists }\end{array}$ & -79 & -58 & -84 & -67 & -89 & -75 & \pm & 5 & 8 & 5 & 8 & 11 & 17 & NS & NS & NS & NS & NS & NS \\
\hline $\begin{array}{l}\text { Social: Talk to } \\
\text { PWS }\end{array}$ & 95 & 92 & 100 & 100 & 100 & 100 & + & 5 & 8 & $\underline{0}$ & $\underline{0}$ & 5 & 8 & NS & NS & $\sim$ & $\sim$ & NS & NS \\
\hline $\begin{array}{l}\text { Social: Friends } \\
\text { with PWS }\end{array}$ & 95 & 100 & 100 & 100 & 100 & 100 & + & 5 & $\underline{0}$ & $\underline{0}$ & $\underline{0}$ & 5 & $\underline{0}$ & NS & $\sim$ & $\sim$ & $\sim$ & NS & $\sim$ \\
\hline $\begin{array}{l}\text { Social: Date } \\
\text { PWS }\end{array}$ & 47 & 38 & 68 & 58 & 58 & 63 & + & 21 & 21 & 11 & 4 & 11 & 25 & NS & NS & NS & NS & NS & NS \\
\hline $\begin{array}{l}\text { Social: Marry } \\
\text { PWS }\end{array}$ & 32 & 21 & 58 & 42 & 58 & 46 & + & 26 & 21 & $\underline{0}$ & 4 & 26 & 25 & NS & NS & NS & NS & NS & NS \\
\hline \multicolumn{20}{|c|}{ SUMMARY } \\
\hline $\begin{array}{l}\text { Number of } \\
\text { "Positive } \\
\text { Change" }\end{array}$ & -- & -- & -- & -- & -- & -- & -- & 30 & 38 & 25 & 26 & 34 & 41 & -- & -- & -- & -- & -- & -- \\
\hline
\end{tabular}


Changing Attitudes 111

\begin{tabular}{|c|c|c|c|c|c|c|c|c|c|c|c|c|c|c|c|c|c|c|c|}
\hline $\begin{array}{l}\text { Number of } \\
\text { "Negative } \\
\text { Change" }\end{array}$ & -- & -- & -- & -- & -- & -- & -- & 14 & 8 & 15 & 17 & 13 & 1 & -- & -- & -- & -- & -- & -- \\
\hline $\begin{array}{l}\text { Number of } \\
\text { "No" Change }\end{array}$ & -- & -- & -- & -- & -- & -- & -- & 7 & 5 & 11 & 8 & 4 & 1 & -- & -- & -- & -- & -- & -- \\
\hline $\begin{array}{l}\text { Number of } \\
\text { "Ambiguous" } \\
\text { Change }\end{array}$ & -- & -- & -- & -- & -- & -- & -- & 11 & 11 & 11 & 11 & 11 & 11 & -- & -- & -- & -- & -- & -- \\
\hline $\begin{array}{l}\text { Number of } \\
\text { significant } \\
\text { differences }\end{array}$ & -- & -- & -- & -- & -- & -- & -- & -- & -- & -- & -- & -- & -- & 1 & 7 & 3 & 2 & 8 & 11 \\
\hline
\end{tabular}


Appendix 7. Comparisons of pre versus post means, pre versus post mean differences and t test probabilities for honors and regular classes PRE-L versus POST-L. The direction of change column is in terms of "positive," "negative," "ambiguous" and "no

change," "Positive" changes are seen as bold, "negative" changes are seen as not bold, "ambiguous" changes are seen as italics, and "no change" items are seen as underlined.

\begin{tabular}{|c|c|c|c|c|c|c|c|c|c|}
\hline \multirow[b]{2}{*}{ Column Number } & \multicolumn{2}{|c|}{ Pre Live } & \multicolumn{2}{|c|}{ Post Live } & \multirow{2}{*}{$\begin{array}{l}-- \\
5\end{array}$} & \multirow{2}{*}{$\begin{array}{l}-- \\
6\end{array}$} & \multirow{2}{*}{$\begin{array}{l}-- \\
7\end{array}$} & \multirow{2}{*}{$\begin{array}{l}-- \\
8\end{array}$} & \multirow{2}{*}{$\begin{array}{l}-- \\
9\end{array}$} \\
\hline & 1 & 2 & 3 & 4 & & & & & \\
\hline \multirow[t]{2}{*}{ Sample Size } & 20 & 20 & 20 & 20 & -- & -- & -- & -- & -- \\
\hline & 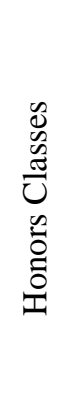 & 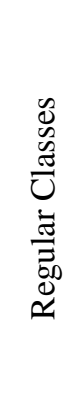 & 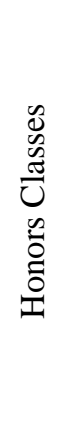 & 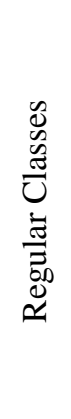 & 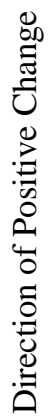 & 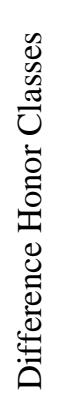 & 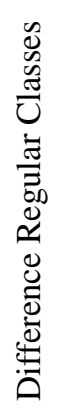 & 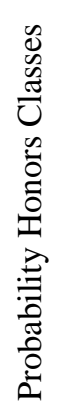 & 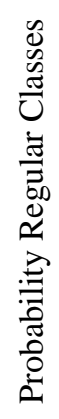 \\
\hline \multicolumn{10}{|l|}{ Demographic Items: } \\
\hline Aspects: Physical health & 65 & 70 & 63 & 75 & \pm & 2 & 5 & NS & NS \\
\hline Aspects: Mental health & 65 & 80 & 55 & 80 & \pm & 10 & 0 & NS & NS \\
\hline Aspects: Learn new things & 73 & 78 & 63 & 80 & \pm & 10 & 3 & NS & $\mathrm{NS}$ \\
\hline Aspects: Speaking ability & 70 & 70 & 68 & 73 & \pm & 3 & 3 & NS & NS \\
\hline \multicolumn{10}{|l|}{ General Items: } \\
\hline Impression: Obese & -15 & -30 & -5 & -28 & + & 10 & 3 & NS & NS \\
\hline Impression: Left handed & 32 & 18 & 29 & 10 & + & 3 & 7 & NS & NS \\
\hline Impression: Stuttering & 20 & -8 & 48 & 15 & + & 28 & 23 & NS & NS \\
\hline Impression: Mentally ill & 10 & -18 & 11 & -15 & + & 1 & 3 & NS & NS \\
\hline Impression: Intelligent & 63 & 73 & 55 & 65 & + & 8 & 8 & NS & NS \\
\hline Want: Obese & -95 & -100 & -88 & -98 & + & 8 & 3 & NS & NS \\
\hline Want: Left handed & -8 & 20 & 11 & 10 & + & 18 & 10 & NS & NS \\
\hline Want: Stuttering & -80 & -73 & -35 & -50 & + & 45 & 23 & $*$ & NS \\
\hline Want: Mentally ill & -90 & -95 & -71 & -85 & + & 19 & 10 & NS & NS \\
\hline Want : Intelligent & 75 & 80 & 73 & 70 & + & 2 & 10 & NS & NS \\
\hline Knowledge: Obese & 8 & 18 & 8 & 33 & + & $\underline{0}$ & 15 & NS & NS \\
\hline
\end{tabular}




\begin{tabular}{|c|c|c|c|c|c|c|c|c|c|}
\hline Knowledge: Left handed & -8 & 3 & 3 & -5 & + & 11 & 8 & NS & NS \\
\hline Knowledge: Stuttering & -55 & -28 & 30 & 15 & + & 85 & 43 & **** & $*$ \\
\hline Knowledge: Mentally ill & -23 & -13 & -10 & -18 & + & 13 & 6 & NS & NS \\
\hline Knowledge: Intelligent & 39 & 43 & 45 & 44 & + & 5 & 2 & NS & NS \\
\hline \multicolumn{10}{|l|}{ Detailed Items: } \\
\hline PWS: Should hide their stuttering & -80 & -55 & -95 & -70 & - & 15 & 15 & NS & NS \\
\hline PWS: Should have important jobs & 47 & 25 & 50 & 55 & + & 3 & 30 & NS & NS \\
\hline PWS: Nervous or Excitable & 20 & 35 & -85 & -55 & - & 105 & 90 & $* * *$ & $* *$ \\
\hline PWS: Shy or fearful & 0 & 30 & -90 & -40 & - & 90 & 70 & $* *$ & NS \\
\hline $\begin{array}{l}\text { PWS: Should blame self for } \\
\text { stuttering }\end{array}$ & -95 & -80 & -100 & -100 & - & 5 & 20 & NS & NS \\
\hline PWS: Can make friends & 90 & 100 & 100 & 100 & + & 10 & $\underline{0}$ & NS & $* * *$ \\
\hline PWS: Lead normal lives & 100 & 90 & 100 & 100 & + & $\underline{0}$ & 10 & $\sim$ & NS \\
\hline PWS: Can do any job they want & 100 & 60 & 80 & 70 & + & 20 & 10 & NS & NS \\
\hline PWS: Difficult to listen to & 0 & -10 & -35 & -25 & - & 35 & 15 & NS & NS \\
\hline Concern: Doctor & -40 & 10 & -50 & -20 & - & 10 & 30 & NS & NS \\
\hline Concern: Neighbor & -75 & -85 & -95 & -85 & - & 20 & $\underline{0}$ & NS & NS \\
\hline Concern: Brother or sister & -35 & 5 & -65 & -30 & - & 30 & 35 & NS & NS \\
\hline Concern: Mom or dad & -55 & 25 & -75 & -30 & - & 20 & 55 & NS & NS \\
\hline Concern: Myself & 10 & 40 & -45 & -10 & - & 55 & 50 & NS & NS \\
\hline Do: Ignore stuttering & 95 & 90 & 90 & 75 & + & 5 & 15 & NS & NS \\
\hline Do: Joke about stuttering & -85 & -90 & -100 & -85 & - & 15 & 5 & NS & NS \\
\hline Do: Fill in words & -50 & -10 & -95 & -75 & - & 45 & 65 & NS & $*$ \\
\hline Do: Feel impatient & -85 & -40 & -100 & -80 & - & 15 & 40 & NS & NS \\
\hline Do: Feel comfortable & 85 & 40 & 90 & 35 & + & 5 & 5 & NS & NS \\
\hline Do: Feel pity & -5 & 30 & -45 & -5 & - & 40 & 35 & NS & NS \\
\hline Do: Say "slow down/Relax" & -35 & -25 & -80 & -75 & - & 45 & 50 & NS & NS \\
\hline Cause: Genetics & 15 & -10 & 75 & 80 & + & 60 & 90 & $*$ & $* *$ \\
\hline Cause: Ghosts, demons, spirits & -100 & -100 & -100 & -100 & - & $\underline{0}$ & $\underline{0}$ & $\sim$ & $\sim$ \\
\hline Cause: Mental/Physical Abuse & -40 & 0 & -100 & -25 & - & 60 & 25 & $*$ & NS \\
\hline Cause: Trying to talk, think too fast & 5 & 60 & -100 & -10 & - & 105 & 70 & $* *$ & $*$ \\
\hline
\end{tabular}




\begin{tabular}{|c|c|c|c|c|c|c|c|c|c|}
\hline Cause: Frightening event & -45 & 10 & -90 & -40 & - & 45 & 50 & NS & NS \\
\hline Cause: act of God & -35 & -80 & -50 & -70 & - & 15 & 10 & NS & NS \\
\hline Cause: Learning or habits & -25 & 50 & -100 & -55 & - & 75 & 105 & $*$ & $* * *$ \\
\hline Cause: Virus or disease & -65 & -32 & -100 & -95 & - & 35 & 63 & NS & $*$ \\
\hline Help: By PWS & -15 & 10 & 25 & 30 & + & 40 & 20 & NS & NS \\
\hline Help: By SLP & 80 & 95 & 95 & 70 & + & 15 & 25 & NS & NS \\
\hline Help: By doctor & 0 & 0 & -20 & -25 & - & 20 & 25 & NS & NS \\
\hline Info: From PWS & -25 & 74 & 90 & 70 & \pm & 115 & 4 & $* *$ & NS \\
\hline Info: Personal Experience & -50 & 0 & -35 & -10 & \pm & 15 & 10 & NS & NS \\
\hline Info: TV, Radio, Films & 60 & 11 & 20 & -30 & \pm & 40 & 41 & NS & NS \\
\hline Info: Magazines, newspapers, books & -30 & -53 & -20 & -40 & \pm & 10 & 13 & NS & NS \\
\hline Info: Internet & -30 & -84 & -50 & -70 & \pm & 20 & 14 & NS & NS \\
\hline Info: School & -20 & 5 & 30 & 40 & \pm & 50 & 35 & NS & NS \\
\hline Info: Doctors, nurses, specialists & -80 & -63 & -75 & -60 & \pm & 5 & 3 & NS & NS \\
\hline Social: Talk to PWS & 85 & 100 & 100 & 90 & + & 15 & 10 & NS & NS \\
\hline Social: Friends with PWS & 95 & 75 & 100 & 90 & + & 5 & 15 & NS & NS \\
\hline Social: Date PWS & 75 & 15 & 85 & 25 & + & 10 & 10 & NS & NS \\
\hline Social: Marry PWS & 61 & 15 & 85 & 10 & + & 24 & 5 & NS & NS \\
\hline \multicolumn{10}{|c|}{ SUMMARY } \\
\hline Number of "Positive Change" & -- & -- & -- & -- & -- & 46 & 37 & -- & -- \\
\hline Number of "Negative Change" & -- & -- & -- & -- & -- & 2 & 11 & -- & -- \\
\hline Number of “No" Change & -- & -- & -- & -- & -- & 3 & 3 & -- & -- \\
\hline Number of “Ambiguous” Change & -- & -- & -- & -- & -- & 11 & 11 & -- & -- \\
\hline Number of significant differences & -- & -- & -- & -- & -- & -- & -- & 9 & 8 \\
\hline
\end{tabular}


Appendix 8. Comparisons of pre versus post means, pre versus post mean differences and $t$ test probabilities for honors and Regular Classes PRE- $V$ versus POST-V, POST-V versus POST-V+L, PRE-V versus POST-V+L. The direction of change column is in terms of "positive," "negative," "ambiguous" and "no change," "Positive" changes are seen as bold, "negative" changes are seen as not bold, "ambiguous" changes are seen as italics, and "no change" items are seen as underlined.

\begin{tabular}{|c|c|c|c|c|c|c|c|c|c|c|c|c|c|c|c|c|c|c|c|}
\hline \multirow{2}{*}{\begin{tabular}{|l} 
Column \\
Number \\
\end{tabular}} & \multicolumn{2}{|c|}{ Pre Video } & \multicolumn{2}{|c|}{ Post Video } & \multicolumn{2}{|c|}{$\begin{array}{c}\text { Post V + } \\
\text { Live }\end{array}$} & \multirow{2}{*}{--} & \multirow{2}{*}{--} & \multirow{2}{*}{--} & \multirow{2}{*}{--} & \multirow{2}{*}{--} & \multirow{2}{*}{--} & \multirow{2}{*}{--} & \multirow{2}{*}{--} & \multirow{2}{*}{--} & \multirow{2}{*}{--} & \multirow{2}{*}{$\begin{array}{c}-- \\
17 \\
\end{array}$} & \multirow{2}{*}{--} & \multirow{2}{*}{--} \\
\hline & 1 & 2 & 3 & 4 & 5 & 6 & & & & & & & & & & & & & \\
\hline \multirow{2}{*}{ Sample Size } & 19 & 24 & 19 & 24 & 19 & 24 & -- & -- & -- & -- & -- & -- & -- & -- & -- & -- & -- & -- & -- \\
\hline & $\begin{array}{l}\tilde{y} \\
0 \\
0 \\
0 \\
0 \\
0 \\
0\end{array}$ & 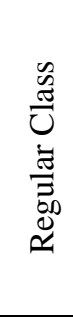 & $\begin{array}{l}\tilde{y} \\
0 \\
0 \\
0 \\
0 \\
0 \\
0\end{array}$ & 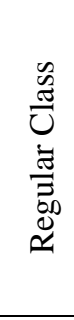 & 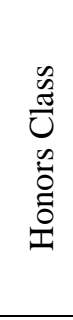 & 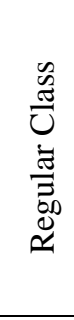 & 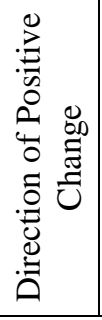 & 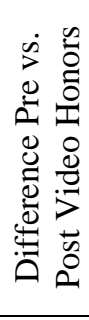 & 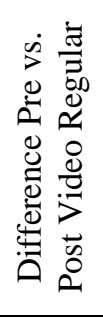 & 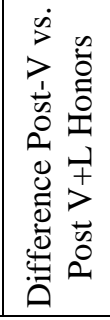 & 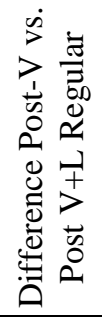 & 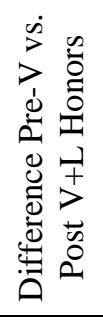 & 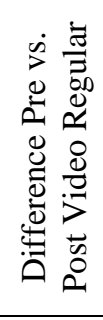 & 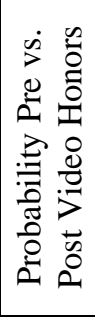 & 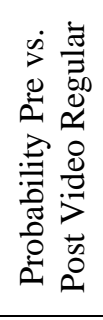 & 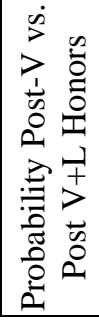 & 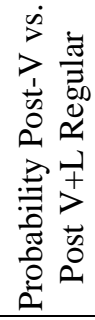 & 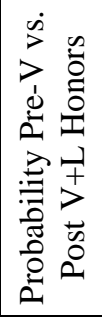 & 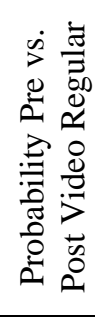 \\
\hline \multicolumn{20}{|l|}{$\begin{array}{l}\text { Demographic } \\
\text { Items: }\end{array}$} \\
\hline $\begin{array}{l}\text { Aspects: } \\
\text { Physical health }\end{array}$ & 61 & 54 & 50 & 58 & 58 & 60 & \pm & 11 & 4 & 8 & 2 & 3 & 6 & NS & NS & NS & NS & NS & NS \\
\hline $\begin{array}{l}\text { Aspects: } \\
\text { Mental health }\end{array}$ & 47 & 66 & 39 & 64 & 50 & 70 & \pm & 8 & 2 & 11 & 6 & 3 & 4 & NS & NS & NS & NS & NS & NS \\
\hline $\begin{array}{l}\text { Aspects: Learn } \\
\text { new things }\end{array}$ & 69 & 74 & 67 & 72 & 67 & 76 & \pm & 3 & 2 & 0 & 4 & 3 & 2 & NS & NS & NS & NS & NS & NS \\
\hline $\begin{array}{l}\text { Aspects: } \\
\text { Speaking } \\
\text { ability }\end{array}$ & 69 & 76 & 69 & 64 & 64 & 66 & \pm & 0 & 12 & 6 & 2 & 6 & 10 & NS & NS & NS & NS & NS & NS \\
\hline \multicolumn{20}{|l|}{ General Items: } \\
\hline $\begin{array}{l}\text { Impression: } \\
\text { Obese }\end{array}$ & -22 & -30 & -31 & -12 & -42 & -20 & + & 8 & 18 & 11 & 8 & 19 & 10 & NS & NS & NS & NS & NS & NS \\
\hline $\begin{array}{l}\text { Impression: } \\
\text { Left handed }\end{array}$ & 56 & 24 & 42 & 40 & 42 & 13 & + & 14 & 16 & $\underline{0}$ & 28 & 14 & 12 & NS & NS & $\sim$ & NS & NS & NS \\
\hline $\begin{array}{l}\text { Impression: } \\
\text { Stuttering }\end{array}$ & 6 & 2 & 33 & 24 & 11 & 10 & + & 28 & 22 & 22 & 14 & 6 & 8 & $*$ & NS & NS & NS & NS & NS \\
\hline $\begin{array}{l}\text { Impression: } \\
\text { Mentally ill }\end{array}$ & -6 & 0 & -8 & 10 & -6 & -4 & + & 3 & 10 & 3 & 14 & $\underline{0}$ & 4 & NS & NS & NS & NS & NS & NS \\
\hline $\begin{array}{l}\text { Impression: } \\
\text { Intelligent }\end{array}$ & 64 & 60 & 78 & 70 & 78 & 64 & + & 14 & 10 & $\underline{0}$ & 6 & 14 & 4 & NS & NS & NS & NS & NS & NS \\
\hline
\end{tabular}


Changing Attitudes 116

\begin{tabular}{|c|c|c|c|c|c|c|c|c|c|c|c|c|c|c|c|c|c|c|c|}
\hline Want: Obese & -97 & -100 & -94 & -98 & -97 & -92 & + & 3 & 2 & 3 & 6 & $\underline{0}$ & 8 & NS & NS & NS & NS & NS & $\mathrm{NS}$ \\
\hline $\begin{array}{l}\text { Want: Left } \\
\text { handed }\end{array}$ & 22 & -4 & 28 & -2 & 17 & 0 & + & 6 & 2 & 11 & 2 & 6 & 4 & NS & NS & NS & NS & NS & NS \\
\hline $\begin{array}{l}\text { Want: } \\
\text { Stuttering }\end{array}$ & -72 & -96 & -42 & -66 & -44 & -54 & + & 31 & 30 & 3 & 12 & 28 & 42 & $*$ & $* *$ & NS & NS & NS & $* * *$ \\
\hline $\begin{array}{l}\text { Want: Mentally } \\
\text { ill }\end{array}$ & -83 & -94 & -72 & -92 & -78 & -88 & + & 11 & 2 & 6 & 4 & 6 & 6 & NS & NS & NS & NS & NS & NS \\
\hline $\begin{array}{l}\text { Want : } \\
\text { Intelligent }\end{array}$ & 92 & 94 & 81 & 88 & 72 & 80 & + & 11 & 6 & 8 & 8 & 19 & 14 & NS & NS & NS & NS & NS & NS \\
\hline $\begin{array}{l}\text { Knowledge: } \\
\text { Obese }\end{array}$ & 8 & 4 & -3 & 2 & -11 & 0 & + & 11 & 2 & 8 & 2 & 19 & 4 & NS & NS & NS & NS & NS & NS \\
\hline $\begin{array}{l}\text { Knowledge: } \\
\text { Left handed }\end{array}$ & -3 & -20 & -3 & -12 & -12 & -22 & + & $\underline{0}$ & 8 & 9 & 10 & 9 & 2 & NS & NS & NS & NS & NS & NS \\
\hline $\begin{array}{l}\text { Knowledge: } \\
\text { Stuttering }\end{array}$ & -47 & -48 & -11 & -24 & -6 & -4 & + & 36 & 24 & 6 & 20 & 42 & 44 & $*$ & NS & NS & NS & NS & $*$ \\
\hline $\begin{array}{l}\text { Knowledge: } \\
\text { Mentally ill }\end{array}$ & -28 & -24 & -24 & -18 & -25 & -24 & + & 4 & 6 & 1 & 6 & 3 & $\underline{0}$ & NS & NS & NS & NS & NS & NS \\
\hline $\begin{array}{l}\text { Knowledge: } \\
\text { Intelligent }\end{array}$ & 31 & 52 & 31 & 30 & 31 & 30 & + & $\underline{0}$ & 22 & $\underline{0}$ & $\underline{0}$ & $\underline{0}$ & 22 & NS & NS & NS & NS & NS & NS \\
\hline \multicolumn{20}{|l|}{$\begin{array}{l}\text { Detailed } \\
\text { Items: }\end{array}$} \\
\hline $\begin{array}{l}\text { PWS: Should } \\
\text { hide their } \\
\text { stuttering }\end{array}$ & -83 & -44 & -100 & -88 & -100 & -96 & - & 17 & 44 & $\underline{0}$ & 8 & 17 & 52 & NS & NS & $\sim$ & NS & NS & $*$ \\
\hline $\begin{array}{l}\text { PWS: Should } \\
\text { have important } \\
\text { jobs }\end{array}$ & 61 & 20 & 89 & 56 & 89 & 68 & + & 28 & 36 & $\underline{0}$ & 12 & 28 & 48 & NS & $*$ & $\sim$ & NS & NS & $*$ \\
\hline $\begin{array}{l}\text { PWS: Nervous } \\
\text { or Excitable }\end{array}$ & 22 & 16 & 0 & 36 & -44 & -32 & - & 22 & 20 & 44 & 68 & 67 & 48 & NS & NS & NS & $*$ & NS & $*$ \\
\hline $\begin{array}{l}\text { PWS: Shy or } \\
\text { fearful }\end{array}$ & 28 & 16 & 0 & 16 & -44 & -16 & - & 28 & $\underline{0}$ & 44 & 32 & 72 & 32 & NS & NS & NS & NS & $*$ & NS \\
\hline $\begin{array}{l}\text { PWS: Should } \\
\text { blame self for } \\
\text { stuttering }\end{array}$ & -78 & -72 & -100 & -96 & -89 & -100 & - & 22 & 24 & 11 & 4 & 11 & 28 & NS & NS & NS & NS & NS & NS \\
\hline $\begin{array}{l}\text { PWS: Can } \\
\text { make friends }\end{array}$ & 100 & 100 & 100 & 100 & 100 & 100 & + & $\underline{0}$ & $\underline{0}$ & $\underline{0}$ & $\underline{0}$ & $\underline{0}$ & $\underline{0}$ & $\sim$ & $\sim$ & $\sim$ & $\sim$ & $\sim$ & $\sim$ \\
\hline $\begin{array}{l}\text { PWS: Lead } \\
\text { normal lives }\end{array}$ & 100 & 96 & 89 & 80 & 100 & 96 & + & 11 & 16 & 11 & 16 & $\underline{0}$ & $\underline{0}$ & NS & NS & NS & NS & NS & $\sim$ \\
\hline PWS: Can do & 83 & 68 & 78 & 88 & 100 & 88 & + & 6 & 20 & 22 & $\underline{0}$ & 17 & 20 & NS & $\mathrm{NS}$ & $\mathrm{NS}$ & $\sim$ & $\mathrm{NS}$ & $\mathrm{NS}$ \\
\hline
\end{tabular}


Changing Attitudes 117

\begin{tabular}{|c|c|c|c|c|c|c|c|c|c|c|c|c|c|c|c|c|c|c|c|}
\hline $\begin{array}{l}\text { any job they } \\
\text { want }\end{array}$ & & & & & & & & & & & & & & & & & & & \\
\hline $\begin{array}{l}\text { PWS: Difficult } \\
\text { to listen to }\end{array}$ & 11 & -20 & -67 & -40 & -83 & -64 & - & 78 & 20 & 17 & 24 & 94 & 44 & $*$ & NS & NS & NS & $* *$ & NS \\
\hline $\begin{array}{l}\text { Concern: } \\
\text { Doctor }\end{array}$ & -33 & -64 & -72 & -64 & -61 & -72 & - & 39 & $\underline{0}$ & 11 & 8 & 28 & 8 & NS & NS & NS & NS & NS & NS \\
\hline $\begin{array}{l}\text { Concern: } \\
\text { Neighbor }\end{array}$ & -94 & -88 & -100 & -88 & -100 & -92 & - & 6 & $\underline{0}$ & $\underline{0}$ & 4 & 6 & 4 & NS & $\sim$ & $\sim$ & NS & NS & NS \\
\hline $\begin{array}{l}\text { Concern: } \\
\text { Brother or sister }\end{array}$ & -72 & -28 & -89 & -44 & -72 & -56 & - & 17 & 16 & 17 & 12 & $\underline{0}$ & 28 & NS & NS & NS & NS & NS & NS \\
\hline $\begin{array}{l}\text { Concern: Mom } \\
\text { or dad }\end{array}$ & -61 & -20 & -89 & -48 & -72 & -56 & - & 28 & 28 & 17 & 8 & 11 & 36 & NS & NS & NS & NS & NS & NS \\
\hline $\begin{array}{l}\text { Concern: } \\
\text { Myself }\end{array}$ & 28 & 20 & -22 & 4 & -11 & 28 & - & 50 & 16 & 11 & 24 & 39 & 8 & NS & NS & NS & NS & NS & NS \\
\hline $\begin{array}{l}\text { Do: Ignore } \\
\text { stuttering }\end{array}$ & 89 & 92 & 78 & 88 & 100 & 80 & + & 11 & 4 & 22 & 8 & 11 & 12 & NS & NS & NS & NS & NS & NS \\
\hline $\begin{array}{l}\text { Do: Joke about } \\
\text { stuttering }\end{array}$ & -94 & -100 & -89 & -100 & -83 & -100 & - & 6 & $\underline{0}$ & 6 & $\underline{0}$ & 11 & $\underline{0}$ & NS & $\sim$ & NS & $\sim$ & NS & $\sim$ \\
\hline $\begin{array}{l}\text { Do: Fill in } \\
\text { words }\end{array}$ & -67 & -48 & -89 & -24 & -83 & -48 & - & 22 & 24 & 6 & 24 & 17 & $\underline{0}$ & NS & NS & NS & NS & NS & NS \\
\hline $\begin{array}{l}\text { Do: Feel } \\
\text { impatient }\end{array}$ & -56 & -60 & -78 & -76 & -89 & -76 & - & 22 & 16 & 11 & $\underline{0}$ & 33 & 16 & NS & NS & NS & NS & NS & NS \\
\hline $\begin{array}{l}\text { Do: Feel } \\
\text { comfortable }\end{array}$ & 33 & 20 & 72 & 67 & 56 & 76 & + & 39 & 47 & 17 & 9 & 22 & 56 & NS & $*$ & NS & NS & NS & $*$ \\
\hline Do: Feel pity & -11 & 0 & -39 & 0 & -61 & -20 & - & 28 & $\underline{0}$ & 22 & 20 & 50 & 20 & NS & NS & NS & NS & NS & $\mathrm{NS}$ \\
\hline $\begin{array}{l}\text { Do: Say "slow } \\
\text { down/Relax" }\end{array}$ & -39 & -16 & -89 & -12 & -88 & -44 & - & 50 & 4 & 1 & 32 & 49 & 28 & NS & NS & $\sim$ & NS & NS & NS \\
\hline $\begin{array}{l}\text { Cause: } \\
\text { Genetics }\end{array}$ & 33 & 36 & -44 & 12 & 44 & 96 & + & 78 & 24 & 89 & 84 & 11 & 60 & $*$ & NS & $*$ & $* *$ & NS & $*$ \\
\hline $\begin{array}{l}\text { Cause: Ghosts, } \\
\text { demons, spirits }\end{array}$ & -100 & -84 & -100 & -84 & -100 & -92 & - & $\underline{0}$ & $\underline{0}$ & $\underline{0}$ & 8 & $\underline{0}$ & 8 & $\sim$ & NS & $\sim$ & NS & NS & NS \\
\hline $\begin{array}{l}\text { Cause: } \\
\text { Mental/Physical } \\
\text { Abuse } \\
\end{array}$ & -44 & -8 & -67 & -28 & -67 & -60 & - & 22 & 20 & $\underline{0}$ & 32 & 22 & 52 & NS & NS & NS & NS & NS & $*$ \\
\hline $\begin{array}{l}\text { Cause: Trying } \\
\text { to talk, think } \\
\text { too fast }\end{array}$ & 17 & 40 & -44 & 20 & -72 & -32 & - & 61 & 20 & 28 & 52 & 89 & 72 & NS & NS & NS & $*$ & $*$ & $* *$ \\
\hline $\begin{array}{l}\text { Cause: } \\
\text { Frightening }\end{array}$ & -17 & 32 & -61 & -24 & -78 & -64 & - & 44 & 56 & 17 & 40 & 61 & 96 & NS & $*$ & NS & $*$ & NS & $* * *$ \\
\hline
\end{tabular}


Changing Attitudes 118

\begin{tabular}{|c|c|c|c|c|c|c|c|c|c|c|c|c|c|c|c|c|c|c|c|}
\hline event & & & & & & & & & & & & & & & & & & & \\
\hline $\begin{array}{l}\text { Cause: act of } \\
\text { God }\end{array}$ & -56 & -48 & -83 & -48 & -78 & -72 & - & 28 & $\underline{0}$ & 6 & 24 & 22 & 24 & NS & NS & NS & NS & NS & NS \\
\hline $\begin{array}{l}\text { Cause: } \\
\text { Learning or } \\
\text { habits }\end{array}$ & 6 & 40 & -67 & 8 & -72 & -60 & - & 72 & 32 & 6 & 68 & 78 & 100 & $*$ & NS & NS & $*$ & $*$ & $* * *$ \\
\hline $\begin{array}{l}\text { Cause: Virus or } \\
\text { disease }\end{array}$ & -83 & -40 & -100 & -64 & -100 & -92 & - & 17 & 24 & $\underline{0}$ & 28 & 17 & 52 & NS & NS & $\sim$ & NS & NS & $*$ \\
\hline Help: By PWS & -17 & -8 & 67 & 80 & 72 & 80 & + & 83 & 88 & 6 & $\underline{0}$ & 89 & 88 & $*$ & $* *$ & NS & NS & $*$ & $* *$ \\
\hline Help: By SLP & 89 & 100 & 94 & 92 & 100 & 88 & + & 6 & 8 & 6 & 4 & 11 & 12 & NS & NS & NS & NS & NS & NS \\
\hline Help: By doctor & 6 & 24 & -11 & 0 & -33 & -8 & - & 17 & 24 & 22 & 8 & 39 & 32 & NS & NS & NS & NS & NS & NS \\
\hline $\begin{array}{l}\text { Info: From } \\
\text { PWS }\end{array}$ & -28 & 16 & -17 & -24 & 33 & 56 & \pm & 11 & 40 & 50 & 80 & 61 & 40 & NS & NS & NS & $*$ & NS & NS \\
\hline $\begin{array}{l}\text { Info: Personal } \\
\text { Experience }\end{array}$ & -56 & -24 & -44 & -52 & -56 & -60 & \pm & 11 & 28 & 11 & 8 & 0 & 36 & NS & NS & NS & NS & NS & NS \\
\hline $\begin{array}{l}\text { Info: TV, } \\
\text { Radio, Films }\end{array}$ & 33 & -4 & 78 & 76 & 78 & 28 & \pm & 44 & 80 & 0 & 48 & 44 & 32 & NS & $* *$ & $\sim$ & NS & NS & NS \\
\hline $\begin{array}{l}\text { Info: } \\
\text { Magazines, } \\
\text { newspapers, } \\
\text { books }\end{array}$ & -39 & -20 & -39 & -36 & -39 & -44 & \pm & 0 & 16 & 0 & 8 & 0 & 24 & $\sim$ & NS & NS & NS & NS & NS \\
\hline Info: Internet & -67 & -60 & -56 & -28 & -67 & -42 & \pm & 11 & 32 & 11 & 14 & 0 & 18 & NS & NS & NS & NS & NS & NS \\
\hline Info: School & -33 & -4 & 0 & 52 & 11 & 68 & \pm & 33 & 56 & 11 & 16 & 44 & 72 & NS & NS & NS & NS & NS & $*$ \\
\hline $\begin{array}{l}\text { Info: Doctors, } \\
\text { nurses, } \\
\text { specialists }\end{array}$ & -78 & -60 & -78 & -72 & -89 & -76 & \pm & 0 & 12 & 11 & 4 & 11 & 16 & $\sim$ & NS & NS & NS & NS & NS \\
\hline $\begin{array}{l}\text { Social: Talk to } \\
\text { PWS }\end{array}$ & 89 & 96 & 100 & 100 & 100 & 100 & + & 11 & 4 & $\underline{0}$ & $\underline{0}$ & 11 & 4 & NS & NS & $\sim$ & $\sim$ & NS & NS \\
\hline $\begin{array}{l}\text { Social: Friends } \\
\text { with PWS }\end{array}$ & 100 & 96 & 100 & 100 & 100 & 100 & + & $\underline{0}$ & 4 & $\underline{0}$ & $\underline{0}$ & $\underline{0}$ & 4 & $\sim$ & NS & $\sim$ & $\sim$ & NS & NS \\
\hline $\begin{array}{l}\text { Social: Date } \\
\text { PWS }\end{array}$ & 39 & 44 & 72 & 56 & 61 & 60 & + & 33 & 12 & 11 & 4 & 22 & 16 & NS & NS & NS & NS & NS & NS \\
\hline $\begin{array}{l}\text { Social: Marry } \\
\text { PWS }\end{array}$ & 33 & 20 & 56 & 44 & 56 & 48 & + & 22 & 24 & $\underline{0}$ & 4 & 22 & 28 & NS & NS & $\sim$ & NS & NS & NS \\
\hline \multicolumn{20}{|c|}{ SUMMARY } \\
\hline $\begin{array}{l}\text { Number of } \\
\text { "Positive } \\
\text { Change" }\end{array}$ & -- & -- & -- & -- & -- & -- & -- & 36 & 30 & 20 & 31 & 36 & 35 & -- & -- & -- & -- & -- & -- \\
\hline
\end{tabular}


Changing Attitudes 119

\begin{tabular}{|c|c|c|c|c|c|c|c|c|c|c|c|c|c|c|c|c|c|c|c|}
\hline $\begin{array}{l}\text { Number of } \\
\text { "Negative } \\
\text { Change" }\end{array}$ & -- & -- & -- & -- & -- & -- & -- & 10 & 13 & 21 & 12 & 7 & 11 & -- & -- & -- & -- & -- & -- \\
\hline $\begin{array}{l}\text { Number of } \\
\text { "No" Change }\end{array}$ & -- & -- & -- & -- & -- & -- & -- & 5 & 8 & 14 & 8 & 8 & 5 & -- & -- & -- & -- & -- & -- \\
\hline $\begin{array}{l}\text { Number of } \\
\text { "Ambiguous" } \\
\text { Change }\end{array}$ & -- & -- & -- & -- & -- & -- & -- & 11 & 11 & 11 & 11 & 11 & 11 & -- & -- & -- & -- & -- & -- \\
\hline $\begin{array}{l}\text { Number of } \\
\text { significant } \\
\text { differences }\end{array}$ & -- & -- & -- & -- & -- & -- & -- & -- & -- & -- & -- & -- & -- & 7 & 6 & 1 & 6 & 5 & 13 \\
\hline
\end{tabular}


Appendix 9. Pilot study versus current study. The direction of change column is in terms of "positive," "negative," "ambiguous" and "no change," "Positive" changes are seen as bold, "negative" changes are seen as not bold, "ambiguous" changes are seen as italics, and "no change" items are seen as underlined.

\begin{tabular}{|c|c|c|c|c|c|c|c|c|c|}
\hline \multirow[b]{2}{*}{ Column Number } & \multicolumn{2}{|c|}{ Pilot Study } & \multicolumn{2}{|c|}{ Current Study } & \multirow{2}{*}{$\begin{array}{l}-- \\
5\end{array}$} & \multirow{2}{*}{$\begin{array}{l}-- \\
6 \\
\end{array}$} & \multirow{2}{*}{$\begin{array}{ll}-- \\
7\end{array}$} & \multirow{2}{*}{$\begin{array}{l}- \\
8\end{array}$} & \multirow{2}{*}{$\begin{array}{l}-- \\
9\end{array}$} \\
\hline & 1 & 2 & 3 & 4 & & & & & \\
\hline \multirow{2}{*}{ Sample Size } & 14 & 26 & 14 & 26 & -- & -- & -- & -- & -- \\
\hline & 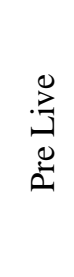 & 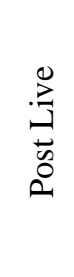 & 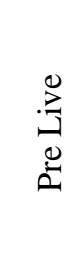 & 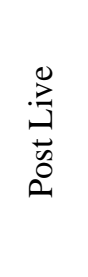 & 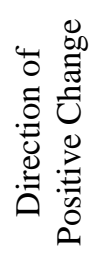 & 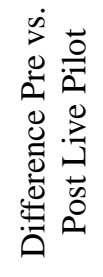 & 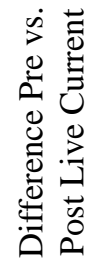 & 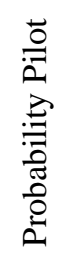 & 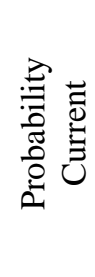 \\
\hline \multicolumn{10}{|l|}{ General Items: } \\
\hline Impression: Obese & -15 & -23 & -1 & -16 & + & 14 & 6 & NS & NS \\
\hline Impression: Left handed & 40 & 24 & 35 & 19 & + & 5 & 5 & NS & NS \\
\hline Impression: Stuttering & 15 & 6 & 21 & 31 & + & 6 & 25 & NS & * \\
\hline Impression: Intelligent & 62 & 68 & 61 & 60 & + & 1 & 7 & NS & NS \\
\hline Want: Obese & -74 & -98 & -78 & -93 & + & 3 & 5 & NS & NS \\
\hline Want: Left handed & 8 & 6 & 9 & 10 & + & 1 & 4 & NS & NS \\
\hline Want: Stuttering & -59 & -76 & -61 & -43 & + & 1 & 34 & NS & $* *$ \\
\hline Want : Intelligent & 84 & 78 & 71 & 71 & + & 13 & 6 & NS & NS \\
\hline Knowledge: Obese & 10 & 13 & 4 & 20 & + & 5 & 8 & NS & NS \\
\hline Knowledge: Left handed & 24 & -3 & 7 & -1 & + & 17 & 1 & NS & NS \\
\hline Knowledge: Stuttering & -46 & -41 & -4 & 23 & + & 42 & 64 & **** & $* * *$ \\
\hline Knowledge: Intelligent & 52 & 41 & 37 & 45 & + & 15 & 4 & NS & NS \\
\hline \multicolumn{10}{|l|}{ Detailed Items: } \\
\hline $\begin{array}{l}\text { PWS: Should hide their } \\
\text { stuttering }\end{array}$ & -58 & -68 & -65 & -83 & - & 7 & 15 & NS & NS \\
\hline $\begin{array}{l}\text { PWS: Should have important } \\
\text { jobs }\end{array}$ & -11 & 36 & -4 & 53 & + & 7 & 17 & NS & NS \\
\hline PWS: Nervous or Excitable & 0 & 28 & -44 & -70 & - & 44 & 98 & $* * *$ & $* * *$ \\
\hline PWS: Shy or fearful & -5 & 15 & -36 & -65 & - & 31 & 80 & NS & $* * *$ \\
\hline PWS: Can make friends & 65 & 95 & 59 & 100 & + & 6 & 5 & NS & NS \\
\hline PWS: Lead normal lives & 58 & 95 & 49 & 100 & + & 9 & 5 & NS & NS \\
\hline PWS: Can do any job they want & 32 & 80 & 24 & 75 & + & 8 & 5 & NS & NS \\
\hline
\end{tabular}




\begin{tabular}{|c|c|c|c|c|c|c|c|c|c|}
\hline Concern: Doctor & 5 & -15 & -56 & -35 & - & 61 & 20 & $* * *$ & NS \\
\hline Concern: Neighbor & -43 & -80 & -11 & -90 & - & 33 & 10 & NS & NS \\
\hline Concern: Brother or sister & 22 & -15 & 18 & -48 & - & 4 & 33 & NS & NS \\
\hline Concern: Myself & 54 & 25 & 40 & -28 & - & 14 & 53 & NS & $*$ \\
\hline Do: Joke about stuttering & -78 & -88 & -83 & -93 & - & 5 & 5 & NS & NS \\
\hline Do: Fill in words & -50 & -30 & -34 & -85 & - & 16 & 55 & NS & $* * *$ \\
\hline Do: Feel impatient & -35 & -63 & -45 & -90 & - & 10 & 28 & NS & NS \\
\hline Do: Feel comfortable & 0 & 63 & 0 & 63 & + & $\underline{0}$ & $\underline{0}$ & NS & NS \\
\hline Do: Feel pity & -11 & 13 & -16 & -25 & - & 4 & 38 & NS & NS \\
\hline Do: Say "slow down/Relax" & -36 & -30 & -58 & -78 & - & 22 & 48 & NS & $*$ \\
\hline Cause: Genetics & 21 & 2 & -9 & 78 & + & 30 & 75 & NS & $* * *$ \\
\hline Cause: Mental/Physical Abuse & 7 & -20 & -37 & -63 & - & 44 & 43 & $* * *$ & $*$ \\
\hline $\begin{array}{l}\text { Cause: Trying to talk, think too } \\
\text { fast }\end{array}$ & 7 & 33 & -19 & -55 & - & 26 & 88 & NS & $* * *$ \\
\hline Cause: Learning or habits & -8 & 13 & -26 & -78 & - & 18 & 90 & NS & $* * *$ \\
\hline Cause: Virus or disease & -33 & -49 & -58 & -97 & - & 24 & 49 & NS & $* *$ \\
\hline Info: From PWS & -39 & 23 & -1 & 80 & \pm & 39 & 57 & $*$ & NS \\
\hline Info: Personal Experience & -58 & -25 & -62 & -23 & \pm & 4 & 2 & NS & NS \\
\hline Info: TV, Radio, Films & -18 & 36 & -40 & -5 & \pm & 22 & 41 & NS & NS \\
\hline $\begin{array}{l}\text { Info: Magazines, newspapers, } \\
\text { books }\end{array}$ & -41 & -41 & -59 & -30 & \pm & 17 & 11 & NS & NS \\
\hline Info: Internet & -65 & -56 & -60 & -60 & \pm & 5 & 4 & NS & NS \\
\hline Info: School & -26 & -8 & -36 & 35 & \pm & 9 & 43 & NS & NS \\
\hline Info: Doctors, nurses, specialists & -50 & -72 & -44 & -68 & \pm & 6 & 4 & NS & NS \\
\hline Social: Talk to PWS & 44 & 93 & 39 & 95 & + & 5 & 3 & NS & NS \\
\hline Social: Friends with PWS & 45 & 85 & 45 & 95 & + & $\underline{0}$ & 10 & NS & NS \\
\hline Social: Date PWS & 1 & 45 & 6 & 55 & + & 6 & 10 & NS & NS \\
\hline Social: Marry PWS & -7 & 45 & -1 & 48 & + & 5 & 2 & NS & NS \\
\hline \multicolumn{10}{|c|}{ SUMMARY } \\
\hline Number of "Positive Change" & -- & -- & -- & -- & -- & 20 & 33 & -- & -- \\
\hline Number of "Negative Change" & -- & -- & -- & -- & -- & 16 & 4 & -- & -- \\
\hline Number of "No" Change & -- & -- & -- & -- & -- & 2 & 1 & -- & -- \\
\hline $\begin{array}{l}\text { Number of “Ambiguous” } \\
\text { Change }\end{array}$ & -- & -- & -- & -- & -- & 7 & 7 & -- & -- \\
\hline $\begin{array}{l}\text { Number of significant } \\
\text { differences }\end{array}$ & -- & -- & -- & -- & -- & -- & -- & 5 & 13 \\
\hline
\end{tabular}


Appendix 10

Respondent evaluations.

\begin{tabular}{|l|}
\hline Honors Class Live Presentation \\
\hline Tim Flynn stutters pretty badly and was actually on MTV’s Show, “True Life” I Stutter.” I got \\
the opportunity to him tell about his life. At first when I saw this guy in our special topics room, \\
I said to myself, "I hope this guy is interesting.” Then he has us do a survey about people who \\
stutter. I was like, “Ok, we are going to learn all about people who stutter.” Once he started to \\
talk, I was in complete SHOCK! I had no idea that this guy actually was going to be stuttering. \\
He started to tell us about his life story, and as he was talking I was inspired. He has to be the \\
bravest individual I possibly know. For what all he has been through, it’s just amazing. For being \\
made fun all his life and coping with the challenges he has to overcome, to survive. As he was \\
telling us his story I was in total awe. If I was in his position, I don’t think I would have been \\
able to do what he is doing now. His going around to schools telling about his story is great. It \\
lets kids know what it's like to have a disorder, and such a unique one at that. I wish Tim could \\
have talked to our class for a lot longer then he did.
\end{tabular}

To me, Tim Flynn has been by far the most influential speaker I have listened too. After I started listening to him for a while, I saw past the stuttering and I saw how normal he really was. I especially enjoyed how he seemed just like us instead of trying to act so professional. It was almost as if I knew him before he came to talk to us, even though I didn't. There was one thing I found quite interesting as Tim talked to us. I thought it was fascinating how he knew when he was going to stutter and when he could get through a whole sentence without having to say it twice. It's almost like there is just a sensor that sets his speech off whenever it feels like it. Tim seems like a truly amazing guy. No matter how hard at times it was for him to talk, he still gave everything he had to talk to our class. Tim is definitely someone that I look up too. I couldn't imagine all of the struggles he went through during his period of growing up. I bet it was hard to hear all the children talk around him, knowing that he can't always do the same. Though I do have to admit, some of the stories he told were quite hilarious. Tim was a very moving speaker through his good stories and bad stories. It's amazing to see how far his speech has come and I truly believe that God gave him this talent to show others that stuttering is something you can overcome.

The speaker really surprised me because I thought he was faking being speech impaired or being a stamee. At first, he carried this on for about ten minutes before I realized he was really a stamee. He really was interesting because on most presentations on normally looses interest but he was able to keep me interested with his funny stories and encounters with cops, he explained how speech impairments work and how one can overcome it. His stories about speech therapists and their methods of trying to help patients was funny to me. Speech therapists telling people to talk in rhythm was ridiculous to me because one would look silly speaking with a rhythm. He taught me how he worked on his speech impairment and is still trying to over it. It's interesting to me how he can tell when he's about to stammer, he talks freely and easily for a while but then stammers for a little while. I was interesting to know why and how that worked. I felt bad for the kids that people think are not smart just because they are stammerers because even though they are smart, people just can't understand them because they stammer. Speech impairments should really be researched so that the population that was it can be treated to their full extent. The speaker really is a happy guy who tries to have fun and has not let his stammering bring him 
down.

Our guest speaker last class, Tim Flynn, was really amazing. To be honest, when Mr. Flynn started talking, his stuttering shocked me. I had no idea stuttering could be that sever. I really enjoyed listening to him share stories about his life. Mr. Flynn was hilarious; he has had some pretty crazy things happen to him. He really made me want to understand how ignorant so many people are towards people with a disability. I can't believe the police seriously thought he was drinking and driving; I thought they, of all people, would know or be able to tell that he had a stutter. Before Mr. Flynn came to our class, I really did not know very much about stuttering. I had experienced some mild, short-term stuttering when I got a concussion freshman year, but nothing bad. Mr. Flynn taught us a lot. Such as, how stuttering played a huge role on his life. He had to say the words that were easy for him, instead of what he wanted to say. After all, fourteen happy meals don't sound very good to me! It upset me how the kids and his teacher made fun of him reading. That's just not right. He's very strong for pushing on through all of that and he has a very bright future ahead of him.

I really didn't know whether this man was faking the stutter at first. I was thinking maybe he will stutter for a while then tell us that's how a person stutters. I really had no idea who he was. Everyone told me he was on True Life: I Stutter afterwards (which I didn't know). I felt very uneasy about the situation, I really thought everyone was going to be rude and laugh. But everyone seem respectful. He seemed to be in a great mood to tell us some of those stories. I guess it was because we were his first period class. He seemed like he really knew that his stuttering was the only way he wanted to talk. I had no idea it was hard for people who stutter to say words that have vowels in them. He also told us that he did not want to talk while taping his finger on a table, I believe that probably annoys him. It was delightful hearing his stories and it really made my day. I told all my friends about the stories and they totally laughed their heads off. He was extremely funny and fine about his situation. I've learned a lot about this guest and about stuttering. I really thought stuttering was caused by being really nervous because I tend to stutter a little bit when I am nervous also when I was little I would stutter when I read in front of class so I felt kind of embarrassed because kids would make fun of me. After years of practice in reading and communicating with others and feeling not so nervous, I didn't seem to repeat words and I made progress making sense. Now that I've learned much and actually met someone who stutters, I feel like I have experienced someone and something new.

When Tim Flynn came to talk to our class at first I didn't know he had a stuttering problem. After hearing him stutter the first time, I was shocked. I didn't know how to react, but I tried to keep looking at him while he was talking like it didn't faze me. I have never met someone with a stuttering problem before. He was really interesting. I couldn't believe it took him three years to find a job. I was happy to hear that Back Bay hired him as a bartender because to be at a job where the people would put you in the back where you couldn't interact with them seems like it would be hard for the person. I know I would probably cry if someone treated me like that, or didn't hire me because I had a stuttering problem. Tim says he takes speech classes. Some of the techniques he learned were really funny. When eh talked about the one where he had to tap on something or put his hand in his pocket and tap I laughed, but I can't imagine meeting someone for the first time that did that while talking, it wouldn't be a very good first impression on a girl. He had a lot of interesting stories and told us a lot of things I never knew about people who stuttered. I'm glad that I got to meet someone with that kind of problem because now when I meet someone else who stutters I wont feel as weird talking to them and I'll know more about what to do and what not to do. 
I had never met a person that stutters before Tim came to speak to us. It was a huge eye-opener to see that he could even tell when it was going to happen. I don't know how well I could deal with wanting to say something, and it not coming out like it sounded in my head. I think it would also be hard to deal with people finishing my sentences. In addition, I didn't know that you could tell when you were going to stutter, for me that would get on my nerves really quick. However, there are a ton of things that you can do to help your stuttering. I thought you just kind of deal with it, but Tim mentioned a ton of things that you could do. I just don't think it could ever be completely cured. I do believe however that hidden disabilities, like that one Tim has, it is truly harder to deal with like he said. When a person has a disability that you can physically see, you know right off the bat that there is something wrong with them. It was a whole new experience for me to see what happens in his daily life and the obstacles he deals with everyday.

When first hearing Tim Flynn begin to talk, I almost thought he was faking it at first. I felt uncomfortable when the presentation first started, and felt bad for him even though he didn't want that at all. Thankfully, a few minutes into him speaking I felt fine, his sense of humor and personality really helped me feel comfortable. The stories Tim told were hilarious! I thought it was good he put these into his presentation because it showed us some of the struggles and confusion he has to deal with on a daily basis just because of his stutter. I don't know how he can deal with the frustration of his stutter at times; it would most likely drive me insane. The way he had to change where he was from was sad, I couldn't believe he did it because of how difficult the word was, but then after he explained what words were hard for him to say and shared that words that started with vowels were the most difficult to say it made sense to me. In conclusion, I really enjoyed this lecture. Tim made it fun, but I also learned so much at the same time. I was glad he was so open and allowed us to ask any questions we wanted to know. He was very nice and calm, and calm made everyone feel comfortable right away which was amazing.

I honestly thought when eh first spoke to us that he was embellishing, or over acting his speech impediment to get his point across quickly. I was shocked, and a little embarrassed when I realized that was just how he spoke. I have heard people stutter before but never that badly, so I felt even more embarrassed and ignorant when he said that his stuttering was not even that awful. Tim really made me see how much more disabling stuttering can be. I realized that speaking and reading can be hard, but he almost got arrested because of it! I know they get frustrated, but I never actually stopped to think how harmful those miscommunications could be. I was really interested in his stories and hearing him talk about how his stuttering progressed throughout his life and his schooling. Even though he has a good sense of humor about it, I was angry about his teacher making it a blatant point to make fun of him. Even though I was taken off guard when he was spoke. He was very enjoyable to listen to and his stories were hilarious and very real. I thought it was really neat how towards the end I barely noticed when he was stuttering. I mean obviously I could tell, but it did not seem awkward or like it was impairing what he was trying to say as much as when I first heard him speak.

Tim was one of the funniest people I have met. I really like the fact that he is trying to be just like everyone else even though he has a stuttering problem. I always thought that people who stutter were really just nervous or just were anxious about something. The stories that Tim told us about his life were hilarious it sucks that the wrong word came out at the wrong time. At first I was a little thrown off because I thought he really didn't stutter anymore, but then he started talking and he still does just not as bad. At least he can tell when it is coming though. It's weird that he can be talking just normally and not even stutter, but then all the sudden he will start starting. It kind of made me sometimes want to finish what he was saying because he would just 
keep saying the same thing over and over again, but I knew if that was me in that situation I wouldn't want people to finish my sentences. I learned a lot from him, and I'm really glad that he could come talk to us about people who stutter because it changed my idea about who stutter so much.

When Tim Flynn first opened his mouth, I was in complete and utter shock. I had no idea that he would actually have a full-blown stutter. Even though before we had to take a survey on stuttering I thought he would just be talking and discussing with us on speech impediments. I didn't believe that he actually had a stutter; I thought he was just impersonating someone with a stutter. But later on, I knew his stutter was legit. At first his stuttering was really bad, but then it got better to where as he could talk with no problem at all! He was very confident and I look up to him for that. As he told us, in his childhood he was ridiculed a lot by fellow students and even teachers! I was very surprised by a teacher making fun of him. I loved all the stories he had told us. It just wasn't some boring presentation. He had given us some very valuable information and I especially liked the one thing he said. Tim had said, "I want people to listen to what I have to say, not how I say it.” I think this is how most people with speech impediments feel.

I think the constant reason I like these guest speakers and people we watch videos on is their attitude. I admire how Tim Flynn is able to use humor to deal with many of the inconveniences his stuttering has. I imagine humor could not be used all the time, but the fact that he can find something funny within a situation probably helps a lot. I like the way he taught about stuttering also. I feel like a lesson that had the potential to be awkward for both him and us listening. However, his ability to just tell stories made it easier for me to be comfortable and learn without him just telling us definitions or giving us notes. I must say that I was one of the many who are unaware of this problem, but now I feel like meeting someone else who stutters wouldn't be a big deal. I think I could talk to them normally without being shocked or confused about what was wrong. I thought of a few questions later in the day I wished I could have asked. I was wondering if people who stutter choose to use sign language or write down what they want. I know this may not be ideal, but wouldn't it have been easier just to put up one or two fingers rather then ordering 14 happy meals? This was just one of my thoughts. Overall, I really enjoyed his lesson.

The other day, in my Special Topics class, I had the opportunity to listen to a very inspiring man speak. This man's name is Timothy Flynn. Who makes Tim so inspiring is that he stutters, but he doesn't even make it a big part of his life. This inspires me because it shows that no matter what you have to deal with in $\mathrm{m}$ life, you can always make it easier for yourself. The class that Tim spoke in was, probably, one of the favorite Special Topics class periods I have ever been in. I will admit, though, that when I first heard Tim speak, it really surprised me. I thought that he was faking it in the beginning because it was happening so frequently. He later told us that when he first starts to speak in front of people, the stuttering happens a lot. I learned that stuttering comes in phases. That means that one moment you will be talking fine and the next the stuttering comes back. Tim also told the class that he can feel when his stuttering is getting ready to happen again. I found this interesting because I wanted to know how he did it. Overall, I thought that Timothy Flynn is a very interesting and funny man. I also learned that his stories are hilarious.

When I was taking the survey about people with a stuttering problem, I didn't think I would actually meet a person with one. It was very unusual because I had never met anyone with one before, and it really startled me when I heard him speak. Even though he said he wasn't nervous, it seemed like he was the whole time because his face was very red and he kept swallowing a lot. After hearing him talk about these things he went through, especially in high school, I really 
wanted to help him somehow. Everything was so easy for me to talk and such, but he had to go through all these years with people thinking he was stupid and retarded and things like that. I can't believe his high school teacher would actually take time out of class to make him read and let everyone laugh at him. Most of all, I can't believe he put up with that kind of behavior from a teacher, but I guess he had too. I can't even imagine how hard it would be to have to talk so long just to talk to someone. People would probably get very annoyed and wouldn't want to listen to me. If they finished my sentences like he said they sometimes do, I would probably get pretty mad because when you want to say something you really don't want someone else to say it for you. I suppose we all have different views, but I only wish that I could make some things easier for people who have a speech problem.

I truly enjoyed the speech Tim gave. When eh first started talking I didn't expect that, but who would. He seemed like a normal college student and one that wouldn't have any problems. But then you heard him talk and I didn't know how to react. After listening to him for a while I realized that he was really cool and just like all of us. His stories were so funny and awesome. I feel like he got his message across really well and knew how to talk to kids. I wish he would have been able to stay longer and talk to us longer. I really enjoyed listening to him and talking to him about his life and childhood. The other thing I didn't understand totally was why he wouldn't try the things that helps him to talk better. I mean I understand when he was like it would be awkward with his hand in his pocket, but you could tap your leg subtly. I know that being a stutterer is something that is a huge part of him, but ht doesn't define him. If he could do something that would help him why not? It would keep him out of all the awkward situations he told us about. All in all though, I really enjoyed that class and loved listening to him.

Tim came into our class to give a presentation on his speech impediment. Tim has a difficult time living a life to the full because he has a stutter. This has affected his life by that he has a hard time meeting new people or doing daily activities such as ordering food or getting a job. In high school, Tim had a teacher who obviously did not understand the emotional strain such a disability puts on a teenagers life because he would point Tim out in front of the entire class. The entire experience of getting to be in the same room as someone with a stutter and getting to really know the real person for who they are rather then just judging people for the disability was a real wake-up call. Everyone in this world is judgmental, and at first, many people did not know how to react to Tim and his stutter, but as class went on we learned that he is very funny and he also has learned to have a sense of humor about his disability about his stutter, but we still know that it is not something that you would ever make fun of him for.

Tim is a very special person. At first I thought his stutter was fake. I was so drawn to his message and his story about how his life has been. With every story he told it brought a valuable lesson to learn. My eyes were focused on him the whole time. I wanted to learn as much as I could. When I first took the survey, I didn't think much about the people who stutter, but then I took the survey again. After Tim spoke and told his story my answers were a little different because after I didn't see stuttering as a disability or a problem, but more so as a gift. Tim taught me many things and I now will never been surprised to hear someone stutter. I took what he said to heart and I'm glad I had the chance to listen to Tim.

\section{Honors Class Video + Live Presentation}

The presentation on speech impairment by Tim Flynn was one that I really, really enjoyed. It may have been my favorite presentation yet! I felt that he was very relatable because he is closer in age to us, because he is from West Virginia, and because he was on a television show that most people my age would watch. Also, he made his presentation really interesting with the 
stories he told us. The one thing that I found most interesting and kind of cool was how he can tell before hand when he is going to and not going to stutter. I'm not quite sure how he can do that but regardless, that is really amazing. I think one of the reasons I found it so cool was because I had always kind of thought that stuttering was more related to certain words and letters. Another thing that I really found interesting was that he was on a television show that I watch. Also, when I saw places in Morgantown that I recognized, like the Beanery or Back Bay, I was really excited because usually you don't see many television shows shot around here. All in all, this was a great presentation and one that I would definitely recommend to try to do again next year.

Tim Flynn ties Mrs. Holehouse as my favorite presenter/presenters. They are both extremely inspirational and favorite funny and personable. I never realized how debilitating a stutter could be. It can be very hindering and annoying, but Tim doesn't see it like that. He kind of just went along with it. It didn't seem to faze him that much; yes, almost every time he talks, he stutters, but he doesn't let it get to him. If it were me, I would get so frustrated and be ticked off and not talk. He doesn't do that he goes around and talks to people about it. He went on national TV talked about it! I don't know how he does it. He is awesome. He seemed like such a good guy, and the way he told the stories and joked about it is a good way I dealing with it I think. But at the same time I feel like that isn't his way of dealing with I think that, that is just the kind of guy he is. His stories were hilarious! I wish he could come back again and talk to use more, and we could ask him questions and stuff. What is the most interesting thing about stuttering is that some sentences he can go on without stuttering at all and then the next 5 minutes he could stutter almost every word. It was like his mind would become lucid for a few moments and go back. It was really cool, and at the same time mind boggling and strange. I want to be a pediatric doctor, and I am almost leaning toward neurology and specifying on stuttering. It is so interesting, and it sounds like a lot of research isn't being done on it, and I think that someone should put some research into. Overall he was a great speaker and a great guy.

I first saw the MTV True Life: I Stutter last year. I thought that it was so neat that someone from our home town was featured on it. When you informed us that Tim Flynn was coming to speak with us, I had no idea it was the same person. I was excited when I figured it out. Never in my life had I heard a person who stutters. I had only seen in on TV and movies. It is so intriguing how Tim told us he can tell when he is about to stutter, yet he has no control over it what-soever. It is amazing how this is such a unique disability, yet doctors have no idea what causes it. I like how Tim addressed the issue of nervousness and stuttering. I always thought that stuttering was something that wasn't caused by nervousness, but it was just made worse by it. Tim explained that he does not stutter because he is nervous, but instead he is nervous because he stutters. He is a wonderful presenter overall. He does not dwell on the fact he stutters, instead embraces it and makes jokes along the way! I truly enjoyed this presentation.

I enjoyed the presentation from Tim Flynn a lot. I really liked his True Life episode on MTV. It really showed me how hard it is to have a stutter. I can only imagine how stressful it must have been to not be able to get a job because people did not want to listen to him. I admire him for sticking it out in school, even though he had to give oral presentations in his classes. I do not know if I would be able to do that because I would be so nervous. I thought it was cool that he was even talking to our class. He has certainly overcome being nervous. I also liked when he was telling about some funny memories of his stuttering. I am glad that he has a good sense of humor about it because it probably helps him not be angry or sad about his problem. If I had a stutter, I would want to be like that because I think it is a good way to deal with it. When he was telling us 
about some of the times his teachers would make fun of him in front of his class, I felt very sorry for him. I can’t imagine why someone would be that cruel. I took a lot from Tim Flynn's presentation. I think that he is really cool, and I am glad he talked to our class.

I was not very familiar with people that stutter, I do not know many people with this disorder. I enjoyed watching the True Life: I Stutter video. I had seen this video in the past, but it was good to view it again because I believe it gives a lot of good information and really shows how these people struggle through certain aspects of their lives. Also, I really liked when Tim spoke, I believe he is the best speaker I've ever had in a class. I was very inspired by him. He seemed to be very comfortable with himself and have a lot of self confidence even with his stuttering. I liked how he didn't “G-rate” the information either, he spoke to us like we were high school students instead of elementary school, as most speakers do. I loved his stories; they were hilarious as well as informational. They showed some of the struggles he's gone through with his social skills and job troubles. The only thing I would change would be for him to talk longer. I think I would have been content with just having him speak for the full hour and half.

Our speaker, Tim Flynn, for stuttering has been my favorite so far. His presentation was very entertaining as well as informing, I learned many things that I was not aware of prior to his presentation and had a great time. The video he showed really made me realize how difficult it can make things beyond just embarrassment. Tim was on the verge of being homeless because he could not get a job and Carolyn had huge emotional strains because she was embarrassed and didn't want anybody to know about her stuttering. I also learned a few things about stuttering. I had always thought that people stuttered worse when they were nervous, which is not true. Time also said some words were harder to say than others when he was stuttering. But he knew when he was going to stutter, and then other times, he can say them perfectly, and he knows he won't stutter for a minute or so. This concept amazes me. I also learned about different ways, Tim doesn't seem so bad now, he just sometimes gets stuck on sounds and repeats them, but the girl who did the pageants seemed to just pause with her mouth open and make a quieter "ahh" sound. I learned a lot from Tim and his presentation to our class, and he was very entertaining. I only it could have been longer.

When reflecting upon the presentation, only one word comes to mind: amazing. I had never seen that episode of True Life, but watching it with Tim actually in the room was incredible. I'm not close with anyone who has a stuttering disorder, and it was so heartbreaking to see how severe something like that can affect a person's life. Each time we have a guest speaker, I am always so thankful for another aspect of my life. Therefore, after Tim's speech, I am reminded how wonderful it is to have good communication skills. Another sensitive place was touch by his visit: people's attitudes towards the disabled. It's wrong how people are so intolerable and rude to those who cannot speak as well as they do. I was frustrated by the treatment of Tim and the two girls on the episode. And when Tim spoke of his freshman year, and his teacher's orders to read aloud for amusement, my heart broke in half. I am going to definitely make it a personal goal to stand up for those who are mistreated, whether it be in school, in my community, or on the street. Tim Flynn was a true inspiration for everyone, especially me.

Tim Flynn was my favorite speaker so far this year. He really kept my interest, and I found his story very interesting. I think the reason I liked him so much is because he is closer to our age and can relate to us, more especially, to our sense of humor. I never really knew very much about stuttering. I always thought that it was just something people do every once in awhile. I also thought that it was cause by nerves. After watching Tim's true life, and hearing him speak, I definitely learned different. People's lives and careers in some cases can be ruined if they have a 
bad stutter. I just do not find it fair that someone can be denied a job just because of the way they speak. People that stutter have the same brain capacity as everyone else so there is no reason that they can not do a job just as well as people who have normal speech. I found Tim to be a hero to other people that stutter. He never gave up no matter what. His whole life he was made fun of and turned down, and he just kept on trucking. He had it set in his mind that he was going to be a bar tender, and he surely did not give up until that dream came true. I find his actions very admirable. I really enjoyed him coming to talk to us and teaching us about stuttering. I learned so much.

When Tim came to talk to our class, I think he did an excellent job. He was very funny and fun to listen to. He came to talk to us about stuttering. If I stuttered I would get so frustrated and probably give up on talking. When we watched the true life video Tim was trying to get a job so he could pay some bills. I think it is awful that some places don't hire people because they have a stutter, or if they do hire them they put them in the back where no one talks to them. I guess I never really thought about that before. In the spring, for my travel soccer team, we were having try outs and that is probably the first time I met someone that had a stutter. Her name was Tiffany and she was a really nice girl. When she talked it didn't really make me think any less of her, I just considered her another girl on the team. Now that I know a little more about stuttering, I am even more excited to play with her this spring. In conclusion, I think it is awful that people with stutters get made fun of and have a lesser change at getting a job. But it is good that they have classes that they can go to and try to improve their stuttering. It has to be frustrating, but they can't give up, they have to keep trying.

If I could describe Tim's presentation in one word, it would have to be WOW! I've never seen that episode of MTV true life before. I never really knew that stuttering could ever be that bad, I just thought it just happened to people because of the way that they talked. Seeing how it truly effected Tim and his life was stunning that people would turn down someone because of how they talk. I think that just by looking at Tim you wouldn't be able to tell that there was anything wrong with him... but as the saying goes, don't judge a book by its cover. Whenever Tim was telling some of his stories, I seriously could not stop laughing. I think that by having a sort of funny outlook on things that happens to him because of his disability really helps him get along with daily life. My favorite story that he told us was about the McDonald's and the 14 happy meals that he had for dinner one night. I am really glad that he hasn't gave up and that he hasn't let what he has hold him back. I would love to hear him speak again and anytime, I really enjoyed it, and I'm happy that he came to talk with us.

Before Tim Flynn came in to speak to our class, I really did not know anything about stuttering, and I definitely did not know it could be so severe. I thought it was really cool to watch True Life: I Stutter and to se Tim in the video, but at the same time in the room. I can't imagine how frustrating it would to have a stutter and not know what the cause of it is and not being able to get rid of it. I thought in Tim's segment of the video that it was very rude of the people to hang up on him when he was trying to get a job. That seemed like it was very hard and very aggravating for Tim. People with a stutter have to overcome many things that people without one take for granted. The three people featured in the video definitely had to go through many obstacles to achieve their goals. I like listening to Tim's point of view about speaking with a stutter and his stories were hilarious. I was glad he could come in and talk to us. I without a doubt learned a lot more, and I could tell after taking each of the three surveys that my answers changed after he spoke to our class.

The episode of "True Life” on stuttering was interesting and eye-opening. I was not aware that 
stuttering could be such a serious problem until we watched that episode in class. The speech impairments of the people in the show had a big effect on their every day lives, and it caused a lot of challenges for them. Although Tim Flynn's portion of the show was the least dramatic, I still thought it was unbelievable that he stuttered so badly. When Tim Flynn talked to our class, I was amazed that he was as normal and comfortable talking to us as he was. Talking in front of a big group of people is nerve-wracking enough when for people who don't stutter, so I can only imagine how scary that would be if you had you had that much trouble talking. I thought Tim Flynn was brave, inspirational, and surprisingly, funny. Telling funny stories was a good idea because it made all of us less tense and more comfortable with Tim's stuttering. I also thought the surveys were interesting. I felt mean and guilty about some of the answers I gave on the first survey after watching the video and listening to Tim talk to our class. I realized that people who have speech impairments are like everyone else and deserve to be treated as normal people.

The presentation by Tim Flynn was very interesting. I had never seen that episode of True Life before, and I really liked it. I used to stutter when I was little but it was never very bad. I felt kind of bad for Tim, because his stuttering keeps him from getting a good job. I think if I was him I would get a job wherever you don't have to talk. I'm not sure where he could have gotten a job, though, because he was still in school and he needed on that paid well. It was nice of that guy to give Tim a job at Back Bay. I never really looked down on people who stutter, and I definitely don't after Tim talked to our class. It would be cool if Tim would come back and talk to our class again, but without the surveys. The surveys took too long and wasted a bit of time. I also thought it was a bit weird that the survey asked what we thought of obese people and people with metal retardations when the presentation was about people that stutter. I think that the presentation was pretty cool, and we should do it again.

Tim Flynn is a very courageous man for speaking publicly about his stuttering. I understand that sometimes it can be frustrating, but he definitely wants to make everyone aware of stuttering and how it isn't a mental or nervous problem, but a neurological issue. I didn't mind taking the surveys from West Virginia University. I think Tim is a courageous man for getting a job in a place where people may be ignorant of stuttering. I also applaud him for sticking through his schooling at WVU and times where he had to speak in class.

In our last special topics class, Tim Flynn came in to speak to us. I had no clued what he was coming to talk to us about. He gave us questionnaires to fill out, and I didn't know what the point to these things were. After we watched the tape of The True Life: I Stutter, I realized what Tim wanted to share with our class. First the tape of The True Life showed three different people who stuttered, one of which was Tim. His stuttering was pretty bad. It was kind of frustrating listening to him talk sometimes. One of the other people that was shown on the tape was even more frustrating. She blocked out letters when she tried to say words. I almost wanted to finish her sentences for her. However, I realized that they can't help it, and they deserve to be treated the as all of us. I was very curious to hear Tim talk to our class. His stuttering was not nearly as bad as it was on the video. Also, he was absolutely hysterical! All of his stories were so entertaining and funny that I couldn't help laughing at everything. He had one of the greatest personalities ever. Also, I thought it was interesting to see that at one point in his talk, he had a hard time saying the name of his hometown. However, later on in his talk, he could say the name of his hometown without stuttering at all. I thought it was strange how that worked. I'm so glad that Tim Flynn could come in to talk to us. He was so funny and entertaining. He made me realize that people who stuttering aren't any different from any of us; they just have a problem to overcome. He inspires me to overcome any challenges that 


\section{Regular Class Live Presentation}

When I first walked into the room, he was talking normally to you so when he started talking to the class and stuttering, I was sort of shocked and let out a little laugh, but then I felt really ignorant and mean. Throughout the whole presentation I could not figure out if he was faking for the survey and an experiment with school or really stuttered. I think Tim is really cool and his stories were entertaining. I now know he really does stutter but that doesn't change who he is or how I think of him.

I thought Tim Flynn's presentation was pretty interesting. It opened my eyes to how frustrating it must be to deal with stuttering. I also saw that people who stutter are completely normal average people.

He has spent a lot of time learning to deal with stuttering. I have a cousin who stutters and he doesn't stutter nearly as much as Tim.

I thought he was funny. The stories he told were funny but I'd have had hated to be in that situation at the time.

The Tim Flynn presentation was pretty sweet. It was different hearing a person stutter like that but after the first minute you don't even notice any more because your so intrigued by the stories he's telling.

I thought it was good. It taught me a lot about stuttering. Tim was smart and funny.

While I was listening to Tim Flynn's presentation, I thought overall he did an excellent job. I listened to all of his stories and was able to not get bored. I was interested in what he was saying. After a bit, I got over the fact that he had a stuttering problem.

To Tim, Hey, I remember you from that time you talked to us. I remember when you told the story about the cockroach. But yea. If you could be any animal what would it be? I think I would be a dragon because I could fly. Also, I feel new respect for people with all speech disorders. You really made a difference. Oh and also, if you were a dragon, would you be a fire dragon or an ice dragon. And who do you think would win in a fight, King Kong or Godzilla. But really, I think Godzilla because he is dinosaur. Well, I had fun.

I think Tim's presentation was good. At first I thought he was just acting, like some other people have done when they're talking to a group about a handicap. When I realized he wasn't it was awkward because I kinda wanted to laugh but I felt bad for him since a lot of people have probably made fun of him or laughed at him before. But he seemed at peace with it \& not upset about what people have thought about his stuttering. He was funny \& I'd probably listen to him again.

I have heard people with mild stutters before but never one that effects their speech that much. It must be frustrating to stutter. I know that I get frustrated when I can't think of the rest of the sentence I was just saying or forget words. I would hate to have to be in school and have to read out loud and stutter. That must have felt embarrassing that people laughed at you.

I thought Tim was a pretty cool person. I loved his stories.

I really thought I would be shocked when I heard him talk but I wasn't. He was just you average person with a little more interesting stories. He seemed awesome and made me want to learn more about stuttering.

I thought his presentation was very informative and that I learned a lot when he was here. I like the way that he gave actual stories about his disorder, not just facts.

I think his presentation was good. The stuttering wasn't really a problem to me at all.

Considering I've had friends with the same problem it was easy for me to adjust. But for the most part I enjoyed it. 
He has obviously spent a lot of time dealing with how to present his problems in the fact that he includes humor in his speech. Speaking in front of a class of strangers - especially teenagers - is hard, I can't even imagine speaking with a stuttering problem. He's turned past embarrassment into bravery.

I thought his presentation really put into perspective what people go through when they stutter. How people react and treat them. His presentation taught me a lot about the subject and how to react and treat others that I meet.

When Tim first started out but then as he kept going I didn't feel as bad because he doesn't sit at home and complain he keeps talking and talking and doesn't seem to care. I really enjoyed his stories too. I thought they were very funny.

Tim's presentation will change my life. He showed and told me a lot about his disorder. I will definitely look differently at people who stutter because Tim's life is very interesting.

Tim, I thought your presentation was very changing. It made me stop and think about how hard your childhood must have been and how frustrated you get. It made me understand how your life is.

I thought Tim Flynn's presentation was cool.

\section{Regular Class Video + Live Presentation}

I felt that Tim Flynn's presentation was very educational, as well as eye opening to the way stuttering effects a person's life.

I found both the video and the presentation you gave very informative and courageous. Stuttering is now a much more real thing to me. I understand more about some of the challenges that people who stutter face, and more of what I can do to help. You clarified some of what I thought were the causes, and made note that the only known contributing factor is genetic.

I thought the True Life video was very informational. I didn't really know much about stuttering and I didn't know how much it can affect people's lives. I had never even known someone who stutters. It was interesting to learn about and to get to know you better. Stutter does happen to people in real life and not just on TV. The video was very helpful. Thanks for showing it to us. The funny stories were also very fun. The stories were a nice bonus to the presentation. Thanks for everything!

I really enjoyed watching the True Life video and Tim's presentation. I thought it was very interesting and I was very interested in what he had to say. Before this, I didn't really encounter anyone with a stuttering problem and I didn't realize his stuttering would be that bad. Over all I really liked it and felt like I learned something about people who stutter.

I thought the True Life video was good. It was very informative and I never really knew what it was like for people that stutter. Now I know a little bit about how hard things like ordering food, getting a job or other things.

Before watching True Life I never realized that stuttering was such a problem. I have previously met stutterers and have a close friend that stutters, but I didn't know that stuttering was that common among the speaking population. Stuttering can be a severe problem, but I do not believe that it is incurable. I think that the tapping finger method, although dull, is a good trick to keep from stuttering. Stutterers, with enough work, can overcome many of their problems and should be able to do any job. Everyone should attempt to help someone that stutters either by comforting them or even by making them talk in conversation.

The presentation was good. I learned a lot about stuttering that I did not know before. It was kind of difficult to understand when he stuttered but he always got out what he wanted to say. I liked the presentation. It was very interesting to hear about something I did not know much about. 
Tim Flynn and the True Life video he was in was very informing and helped me learn about stuttering. I learned that the cause of stuttering may be due to genetics. I realized those who stutter learn techniques that help them live normal lifestyles. Tim Flynn and True Life taught me a lot.

The True Life video was very informing on leading a life with a stuttering disorder. It showed how typical people with the disorder have to go through each day. The video also shows that they can always lead a completely normal life and do things that anybody can do. Although that showed me how difficult it can be to make it with a stuttering disorder.

What I learned from Tim's presentation was that stuttering does not just happen when your nervous but all the time. Stuttering is genetic and some days are better than others. With stuttering some days you could talk really good and other days you could stutter really bad. Tim's presentation was really fun and informative. I learned a lot from his presentation.

I liked the True Life video. I thought it was cool to see how people who stutter live and cope with the problems that occur because of stuttering. I learned from Tim that stuttering is caused by genetics, so if your parents stutter you are more likely to. When people in the video hung up on them I felt mad. That wasn't very nice. I liked hearing Tim tell those funny stories.

I personally enjoyed Tim's presentation on stuttering. I think it brought me a lot of insight to what people who stutter go through on a daily basis. It also was an eye-opener to the little things I take for granted. The episode of True Life was also good to watch because it showed different people and their fight with stuttering. Overall I liked the presentation.

I thought the True Life video was very good and it really made me think more about stuttering. You don't really realize how hard life can be trying to live with it when you're just reading about it in a textbook. Seeing real life examples like this really makes it real. I think more people should watch the True Life video, because I think people very often don't realize how good they have it. They video was easy to connect to. It made stuttering real and not just some condition in a textbook. 
Appendix 11

Live Presentation Script

\begin{tabular}{|c|c|c|}
\hline Minutes & Topics & Descriptions \\
\hline $0-5$ & Introduction & $\begin{array}{l}\text { Author introduces himself and makes } \\
\text { reference to how he avoids saying his } \\
\text { hometown because it is difficult to say. }\end{array}$ \\
\hline \multirow[t]{4}{*}{$5-15$} & Childhood experiences & $\begin{array}{l}\text { Author states his first stuttering memory } \\
\text { from childhood. }\end{array}$ \\
\hline & & $\begin{array}{l}\text { Author tells three childhood school stories, } \\
\text { two from grade school and one from high } \\
\text { school. }\end{array}$ \\
\hline & & $\begin{array}{l}\text { Author then leads into a humorous story } \\
\text { about trying to order fast food. }\end{array}$ \\
\hline & & $\begin{array}{l}\text { Author tells about another humorous story } \\
\text { about how an SLP taught him to tap his } \\
\text { finger to speak. }\end{array}$ \\
\hline $15-25$ & Coping Mechanisms & $\begin{array}{l}\text { Author makes reference to how humor is } \\
\text { his biggest coping mechanism. }\end{array}$ \\
\hline $25-30$ & Cause of stuttering & $\begin{array}{l}\text { A brief summary about the relationship } \\
\text { between genetics and stuttering }\end{array}$ \\
\hline $30-45$ & Personal Stories & $\begin{array}{l}\text { Author tells several other humorous stories } \\
\text { that describe how stuttering is a way of life } \\
\text { and how stuttering has impacted him. }\end{array}$ \\
\hline
\end{tabular}




\section{Instructions}

Dear participant,

Thank you for agreeing to participate in this research project designed to explore public opinion about a number of human attributes and characteristics in various places around the world. In the following attached questionnaire, we ask you to give your honest opinions about five different human attributes and some information about yourself that will help us better interpret the results from many people. We also ask that you provide more detailed opinions about one of the human attributes.

Please do not write your name, address, or telephone number anywhere on the questionnaire or on an envelope used to send it back to us. It is important that we do not know your name. We want to maintain complete confidentiality.

Completely filled-out questionnaires will help us obtain a better picture of public opinion. Nevertheless, as you fill out the questionnaire, you are free to omit any items or stop responding for any reason, without any prejudice or penalty.

The questionnaire asks for a few written short answers and for checking boxes [ $\theta$ ] that apply to you. But mostly it involves making judgments by drawing a circle around your answer. Some of these judgments are numbers on number scales, while others are "Yes," "No," or "Not sure" choices. There are no right or wrong answers! We ask you to work quickly and mark your first impression. Please do not go back and change any of your responses unless you later discover that you did not understand an item or that you answered on the wrong line.

When you circle an answer, be sure to draw a small, circle around the number, "?,” or word that best represents your opinion. On the number scales, you may circle any number, but feel free to mark the extreme negative or positive ends of the scale as well as the exact middle if one of those best shows your opinion. When you check a box, please put a small $\checkmark$ in the box [ $\theta]$.

Following are four examples. The first one shows someone's fairly positive opinion about being tall, the second, a very negative opinion about being short, neutral about wearing glasses, and either has no opinion or knows nothing about wearing a hearing aid.

\begin{tabular}{l|ccccc|c}
\hline $\begin{array}{l}\text { My general impression of a } \\
\text { person who... }\end{array}$ & $\begin{array}{c}\text { Very } \\
\text { negative }\end{array}$ & $\begin{array}{c}\text { Somewhat } \\
\text { negative }\end{array}$ & Neutral & $\begin{array}{c}\text { Somewhat } \\
\text { positive }\end{array}$ & $\begin{array}{c}\text { Very } \\
\text { positive }\end{array}$ & $\begin{array}{c}\text { Not } \\
\text { sure }\end{array}$ \\
\hline is tall & $\mathbf{1}$ & $\mathbf{2}$ & $\mathbf{3}$ & $\mathbf{4}$ & $\mathbf{5}$ & $?$ \\
\hline is short & $\mathbf{1}$ & $\mathbf{2}$ & $\mathbf{3}$ & $\mathbf{4}$ & $\mathbf{5}$ & $?$ \\
\hline wears glasses & $\mathbf{1}$ & $\mathbf{2}$ & 3 & $\mathbf{4}$ & $\mathbf{5}$ & $?$ \\
\hline wears a hearing aid & $\mathbf{1}$ & $\mathbf{2}$ & $\mathbf{3}$ & $\mathbf{4}$ & $\mathbf{5}$ & $?$ \\
\hline
\end{tabular}

Thank you very much for your help. 
PUBLIC OPINION SURVEY OF HUMAN ATTRIBUTES (POSHA)

Please tell about yourself in this section.

\begin{tabular}{l|c|c|c}
\hline Dates: & Month & Day & Year \\
\hline & e.g., January & e.g., 23 & e.g., 2008 \\
Today's date is: & & - & \\
\hline & & & \\
\hline
\end{tabular}

\begin{tabular}{l|l|l|l}
\hline $\begin{array}{c}\text { Residence and } \\
\text { Citizenship }\end{array}$ & Country & State (or Province) & $\begin{array}{c}\text { City (or Town, } \\
\text { Village, Region) }\end{array}$ \\
\hline I now live in: & & & \\
\hline I was born in: & & & \\
\hline
\end{tabular}

Check $[\checkmark]$ all that apply

I am: Male [ ] Female [ ]

Class Ranking: Freshman [ ] Sophomore [ ] Junior [ ] Senior [ ]

My native language is:

I can also easily understand and speak the following languages:

1.

2.

3.

My race is:

My religion is:

\begin{tabular}{|c|c|c|c|c|c|c|}
\hline $\begin{array}{l}\text { I would rate the following } \\
\text { aspects of my life now as... }\end{array}$ & $\begin{array}{l}\text { Very } \\
\text { poor }\end{array}$ & Poor & Average & Good & Excellent & $\begin{array}{l}\text { Not } \\
\text { sure }\end{array}$ \\
\hline my physical health & 1 & 2 & 3 & 4 & 5 & $?$ \\
\hline my mental health & 1 & 2 & 3 & 4 & 5 & ? \\
\hline my ability to learn new things & 1 & 2 & 3 & 4 & 5 & ? \\
\hline my speaking ability & 1 & 2 & 3 & 4 & 5 & ? \\
\hline
\end{tabular}




\section{Now, please give us your opinions about people with all the characteristics listed.}

\begin{tabular}{l|ccccc|c}
\hline $\begin{array}{l}\text { My overall impression of a } \\
\text { person who... }\end{array}$ & $\begin{array}{c}\text { Very } \\
\text { negative }\end{array}$ & $\begin{array}{c}\text { Somewhat } \\
\text { negative }\end{array}$ & Neutral & $\begin{array}{c}\text { Somewhat } \\
\text { positive }\end{array}$ & $\begin{array}{c}\text { Very } \\
\text { positive }\end{array}$ & $\begin{array}{c}\text { Not } \\
\text { sure }\end{array}$ \\
\hline is obese (much overweight) & -2 & -1 & 0 & +1 & +2 & $?$ \\
\hline is left handed & -2 & -1 & 0 & +1 & +2 & $?$ \\
\hline has a stuttering disorder & -2 & -1 & 0 & +1 & +2 & $?$ \\
\hline is mentally ill & -2 & -1 & $\mathbf{0}$ & +1 & +2 & $?$ \\
\hline is intelligent & -2 & -1 & 0 & +1 & +2 & $?$ \\
\hline
\end{tabular}

\begin{tabular}{l|ccccc|c}
\hline $\begin{array}{l}\text { I would want to be a person } \\
\text { who... }\end{array}$ & $\begin{array}{c}\text { Strongly } \\
\text { disagree }\end{array}$ & $\begin{array}{c}\text { Somewhat } \\
\text { disagree }\end{array}$ & Neutral & $\begin{array}{c}\text { Somewhat } \\
\text { agree }\end{array}$ & $\begin{array}{c}\text { Strongly } \\
\text { agree }\end{array}$ & $\begin{array}{c}\text { Not } \\
\text { sure }\end{array}$ \\
\hline is obese (much overweight) & -2 & -1 & 0 & +1 & +2 & $?$ \\
\hline is left handed & -2 & -1 & 0 & +1 & +2 & $?$ \\
\hline has a stuttering disorder & -2 & -1 & 0 & +1 & +2 & $?$ \\
\hline is mentally ill & -2 & -1 & 0 & +1 & +2 & $?$ \\
\hline is intelligent & -2 & -1 & 0 & +1 & +2 & $?$ \\
\hline
\end{tabular}

\begin{tabular}{l|ccccc|c}
\hline $\begin{array}{l}\text { The amount I know about } \\
\text { people who... }\end{array}$ & None & A little & Some & A lot & $\begin{array}{c}\text { A great } \\
\text { deal }\end{array}$ & $\begin{array}{c}\text { Not } \\
\text { sure }\end{array}$ \\
\hline are obese (much overweight) & $\mathbf{1}$ & $\mathbf{2}$ & $\mathbf{3}$ & $\mathbf{4}$ & $\mathbf{5}$ & $\boldsymbol{?}$ \\
\hline are left handed & $\mathbf{1}$ & $\mathbf{2}$ & $\mathbf{3}$ & $\mathbf{4}$ & $\mathbf{5}$ & $\boldsymbol{?}$ \\
\hline have a stuttering disorder & $\mathbf{1}$ & $\mathbf{2}$ & $\mathbf{3}$ & $\mathbf{4}$ & $\mathbf{5}$ & $?$ \\
\hline are mentally ill & $\mathbf{1}$ & $\mathbf{2}$ & $\mathbf{3}$ & $\mathbf{4}$ & $\mathbf{5}$ & $?$ \\
\hline are intelligent & $\mathbf{1}$ & $\mathbf{2}$ & $\mathbf{3}$ & $\mathbf{4}$ & $\mathbf{5}$ & $\boldsymbol{?}$ \\
\hline
\end{tabular}

\begin{tabular}{l|c|c|c|c|c|c}
\hline $\begin{array}{l}\text { Following are people I have } \\
\text { known who... } \\
\text { (Check }[\checkmark \text { ] all that apply) }\end{array}$ & Nobody & $\begin{array}{c}\text { Acquaint- } \\
\text { ance }\end{array}$ & $\begin{array}{c}\text { Close } \\
\text { Friend }\end{array}$ & Relative & Me & Other \\
\hline are obese (much overweight) & $\theta$ & $\theta$ & $\theta$ & $\theta$ & $\theta$ & $\theta$ \\
\hline are left handed & $\theta$ & $\theta$ & $\theta$ & $\theta$ & $\theta$ & $\theta$ \\
\hline has a stuttering disorder & $\theta$ & $\theta$ & $\theta$ & $\theta$ & $\theta$ & $\theta$ \\
\hline is mentally ill & $\theta$ & $\theta$ & $\theta$ & $\theta$ & $\theta$ & $\theta$ \\
\hline is intelligent & $\theta$ & $\theta$ & $\theta$ & $\theta$ & $\theta$ & $\theta$ \\
\hline
\end{tabular}




\section{Now, please give us more detailed opinions about the disorder of stuttering.}

\begin{tabular}{|c|c|c|c|}
\hline \multicolumn{3}{|l|}{ People who stutter... } & \multirow{2}{*}{$\begin{array}{c}\text { Not sure } \\
? ?\end{array}$} \\
\hline should try to hide their stuttering & Yes & No & \\
\hline $\begin{array}{l}\text { should have jobs where they have to correctly understand and decide } \\
\text { important things }\end{array}$ & Yes & No & ? \\
\hline are nervous or excitable & Yes & No & ? \\
\hline are shy or fearful & Yes & No & ? \\
\hline have themselves to blame for their stuttering & Yes & No & ? \\
\hline can make friends & Yes & No & ? \\
\hline can lead normal lives & Yes & No & ? \\
\hline can do any job they want & Yes & No & ? \\
\hline are difficult to listen too & Yes & No & $?$ \\
\hline \multicolumn{3}{|c|}{ If the following people stuttered, I would be concerned or worried... } & Not sure \\
\hline my doctor & Yes & No & $?$ \\
\hline my neighbor & Yes & No & ? \\
\hline my brother or sister & Yes & No & ? \\
\hline *my mom or dad & Yes & No & ? \\
\hline me & Yes & No & ? \\
\hline
\end{tabular}

\begin{tabular}{|c|c|c|c|}
\hline \multicolumn{3}{|l|}{ If I were talking with a person who stutters, I would... } & Not sure \\
\hline try to act like the person was talking normally & Yes & No & ? \\
\hline make a joke about stuttering & Yes & No & ? \\
\hline fill in the person’s words & Yes & No & ? \\
\hline feel impatient (not want to wait while the person stutters) & Yes & No & ? \\
\hline feel comfortable or relaxed & Yes & No & ? \\
\hline feel pity for the person & Yes & No & ? \\
\hline tell the person to "slow down" or "relax" & Yes & No & ? \\
\hline If selected, what? & & & \\
\hline \multicolumn{3}{|l|}{ I believe stuttering is caused by... } & Not sure \\
\hline genetic inheritance & Yes & No & ? \\
\hline ghosts, demons, or spirits & Yes & No & ? \\
\hline being mentally or physically abused & Yes & No & ? \\
\hline trying to think or talk too fast & Yes & No & ? \\
\hline a very frightening event & Yes & No & ? \\
\hline an act of God & Yes & No & ? \\
\hline learning or habits & Yes & No & ? \\
\hline a virus or disease & Yes & No & ? \\
\hline other If selected, what? & Yes & No & ? \\
\hline
\end{tabular}




\begin{tabular}{l|c|c|c}
\hline I believe stuttering should be helped by... & Not sure \\
\hline other people who stutter & Yes & No & ? \\
\hline a speech and language therapist & Yes & No & $?$ \\
\hline a medical doctor & Yes & No & ? \\
\hline
\end{tabular}

\begin{tabular}{|c|c|c|c|}
\hline \multicolumn{3}{|c|}{ My knowledge about stuttering comes from... } & \multirow{2}{*}{$\begin{array}{c}\text { Not sure } \\
?\end{array}$} \\
\hline my experience with other people who stutter & Yes & No & \\
\hline personal experience (me, my family, friends) & Yes & No & ? \\
\hline television, radio, or films & Yes & No & ? \\
\hline magazines, newspapers, or books & Yes & No & ? \\
\hline the Internet & Yes & No & ? \\
\hline school & Yes & No & ? \\
\hline doctors, nurses, or other specialists & Yes & No & ? \\
\hline
\end{tabular}

\begin{tabular}{l|c|c|c}
\hline In a social setting, I would... & Yes & No & Not sure \\
\hline talk to a person who stutters & Yes & No & $?$ \\
\hline be friends with a person who stutters & Yes & No & $?$ \\
\hline date a person who stutters & Yes & No & $?$ \\
\hline marry a person who stutters & \\
\hline
\end{tabular}

Timothy W. Flynn, B.S. Kenneth O. St. Louis, Ph.D. 